\title{
Article
}

\section{Quantum Eberlein compactifications and invariant means}

Daws, Matthew and Das, Biswarup

Available at http://clok.uclan.ac.uk/35996/

Daws, Matthew ORCID: 0000-0003-1707-4308 and Das, Biswarup (2016)

Quantum Eberlein compactifications and invariant means. Indiana University Mathematics Journal, 65 (1). pp. 307-352. ISSN 0022-2518

It is advisable to refer to the publisher's version if you intend to cite from the work.

For more information about UCLan's research in this area go to

http://www.uclan.ac.uk/researchgroups/ and search for < name of research Group>.

For information about Research generally at UCLan please go to

http://www.uclan.ac.uk/research/

All outputs in CLoK are protected by Intellectual Property Rights law, including Copyright law. Copyright, IPR and Moral Rights for the works on this site are retained by the individual authors and/or other copyright owners. Terms and conditions for use of this material are defined in the policies page.

\section{CLoK}

Central Lancashire online Knowledge www.clok.uclan.ac.uk

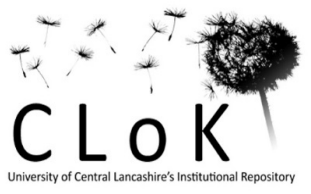




\title{
Quantum Eberlein compactifications and invariant means
}

\author{
Biswarup Das, Matthew Daws
}

June 9, 2018

\begin{abstract}
We propose a definition of a " $C^{*}$-Eberlein" algebra, which is a weak form of a $C^{*}$-bialgebra with a sort of "unitary generator". Our definition is motivated to ensure that commutative examples arise exactly from semigroups of contractions on a Hilbert space, as extensively studied recently by Spronk and Stokke. The terminology arises as the Eberlein algebra, the uniform closure of the Fourier-Stieltjes algebra $B(G)$, has character space $G^{\mathcal{E}}$, which is the semigroup compactification given by considering all semigroups of contractions on a Hilbert space which contain a dense homomorphic image of $G$. We carry out a similar construction for locally compact quantum groups, leading to a maximal $C^{*}$ Eberlein compactification. We show that $C^{*}$-Eberlein algebras always admit invariant means, and we apply this to prove various "splitting" results, showing how the $C^{*}$-Eberlein compactification splits as the quantum Bohr compactification and elements which are annihilated by the mean. This holds for matrix coefficients, but for Kac algebras, we show it also holds at the algebra level, generalising (in a semigroup-free way) results of Godement.

2010 Mathematics Subject Classification: Primary 43A07, 43A60, 46L89, 47D03; Secondary 22D25, 43A30, 43A65, 47L25
\end{abstract}

Keywords: Eberlein compactification, Invariant means, Locally compact quantum groups.

\section{Introduction}

For a locally compact group $G$, there is a tight connection between semigroup compactifications of $G$ and certain introverted subspaces of $C^{b}(G)$. For example, the weakly almost periodic functions on $G$ form a commutative $C^{*}$-algebra wap $(G)$ whose character space $G^{\text {wap }}$ becomes a compact semigroup whose product is jointly continuous. Indeed, $G^{\text {wap }}$ is the largest such "semitopological" semigroup which contains a dense homomorphic image of $G$. For further details see the monograph [4]. It can be hard to describe functions in $\operatorname{wap}(G)$, but any coefficient of a unitary representation, that is, a function in the FourierStieltjes algebra $B(G)$, is weakly almost periodic, and so the uniform norm closure of $B(G)$ in $C^{b}(G)$, the Eberlein algebra $\mathcal{E}(G)$, is a subalgebra of $\operatorname{wap}(G)$. Recent work of Spronk and Stokke, 39], studied $\mathcal{E}(G)$ and its character space $G^{\mathcal{E}}$ in detail. In particular, $G^{\mathcal{E}}$ is the maximal semigroup which arises as a semigroup of contractions on a Hilbert space; see also [31.

In this paper, we are interested in "non-commutative" Eberlein algebras. There has recently been much interest in "topological quantum groups", which we shall take to mean $C^{*}$-bialgebras, probably with additional structure, such as (locally) compact quantum groups. For example, if $G$ is a locally compact group, we consider the algebra $C_{0}(G)$ with the coproduct $\Delta: C_{0}(G) \rightarrow M\left(C_{0}(G) \otimes C_{0}(G)\right) \cong$ $C^{b}(G \times G) ; \Delta(f)(s, t)=f(s t)$ which captures the group product. The theory of locally compact quantum groups, [25], studies such objects where also left and right invariant weights are assumed. Then those quantum groups which arise from a commutative $C^{*}$-algebra are exactly those coming from locally compact groups in this way.

In this paper, we study an analogous "non-commutative" model, which we call a " $C^{*}$-Eberlein algebra", which is a $C^{*}$-algebra with additional structure. Our construction ensures that if we have a commutative $C^{*}$-algebra then we exactly recover a semigroup of contractions on a Hilbert space. We then go on to study compactifications: given a $C^{*}$-bialgebra (maybe with extra structure) what is the 
"maximal" $C^{*}$-Eberlein algebra which contains a dense "homomorphic" copy of the starting bialgebra? We make sense of this, and prove the existence of such an object.

Much of our motivation comes from a longer-term project of seeing what weakly-almost periodic functions (or uniformly continuous, and so forth) might mean in the non-commutative setting. For a locally compact group $G$ one can form the Banach algebra $L^{1}(G)$ and then recover wap $(G)$ by looking at the weakly almost periodic functionals on $L^{1}(G)$. This Banach algebraic definition makes sense in general, and has been studied for quantum groups, see for example [33, Section 4] and [20]. However, to our knowledge there has been little study in terms of compactifications (but compare [10, 35]). Of course, a first step in this programme must be to have an independent model of what a "semitopological quantum semigroup" is. This paper represents a step in this direction, where we can make use of Hilbert space techniques.

A major use of $\operatorname{wap}(G)$ is that it always admits an invariant mean (whereas for $L^{\infty}(G)$ we of course need $G$ to be amenable). For example, this allows us to talk about "mixing" for actions of artibrary groups, not just $\mathbb{Z}$ or $\mathbb{R}$, see [5]. The construction of this mean is typically derived from a fixed-point theorem (compare [4, Chapter 4.2]) but for $\mathcal{E}(G)$ one can instead use Hilbert space techniques. This idea also successfully works for non-commutative $C^{*}$-Eberlein algebras, allowing us to construct invariant means. As an application of this, for Kac algebras (locally compact quantum groups with a particularly well-behaved antipode) we show that the Eberlein compactification splits as a direct sum of the almost periodic compactification and those "functions" which are annihilated by the mean, thus generalising Godemont's Theorem [17, Theorem 16] in the commutative situation.

The paper is organised as follows: we first introduced some terminology and notation. In Section 3 we study semigroups of contractions on a Hilbert space, and show how to think of such objects in a $C^{*}$ algebra framework. We can then allow the $C^{*}$-algebra to be noncommutative, while still maintaining a lot of interesting structure. For example, we study morphisms of such objects in Section 3.6, in Section 3.7 we use some dual Banach algebra techniques to study permanence properties (quotients and subspaces) of these objects, and in Section 3.8 we study which such semigroups arise naturally from a (locally compact) quantum group. In Section 4 we construct invariant means on these algebras, and in Section [5] we use the results of Section 3.8 to construct compactifcations. This has obvious links with quantum group compactifications, see [10, 36], and we explore these links in Section 6. In Section 7, for Kac algebras, we use invariant means to decompose unitary corepresentations into "almost periodic" or "compact vector" parts, and parts which are annihilated by the mean. In the final section, we explain carefully how our results relate to the work of [39], and also quickly look at what happens in the cocommutative case (that is, for the Fourier algebra, instead of for $\left.L^{1}(G)\right)$.

\subsection{Acknowledgements}

The first named author was supported by the EPSRC grant EP/I026819/1 and a WCMS-postdoctoral grant. The 2nd named author was partially supported by the EPSRC grant EP/I026819/1. We thank Adam Skalski and Stuart White for inspiration about the techniques in Section 4 . We thank the anonymous referee for very careful proof-reading.

\section{Notation and setting}

For a Hilbert space $H$ we write $\mathcal{B}(H), \mathcal{B}_{0}(H)$ for the bounded, respectively, compact, operators on $H$. We write $\bar{H}$ for the conjugate Hilbert space: this has set of vectors $\{\bar{\xi}: \xi \in H\}$ with scalar-multiplication such that $H \rightarrow \bar{H}, \xi \mapsto \bar{\xi}$ becomes antilinear. For $x \in \mathcal{B}(H)$ we define $\bar{x} \in \mathcal{B}(\bar{H})$ by $\bar{x}(\bar{\xi})=\overline{x(\xi)}$. We also define $\top: \mathcal{B}(H) \rightarrow \mathcal{B}(\bar{H})$ the "transpose" map by $(\top(x) \bar{\xi} \mid \bar{\eta})=\left(\overline{x^{*} \xi} \mid \bar{\eta}\right)=\left(\eta \mid x^{*} \xi\right)=(x \eta \mid \xi)$ for $\xi, \eta \in H$; we might also write $x^{\top}$ for $\top(x)$.

At certain points we use some basic ideas from the theory of Operator Spaces, especially the various natural tensor products, see [15, Section II] or [32, Part I]. Similarly, we freely use the concept of the 
multiplier algebra of a $C^{*}$-algebra, see [29, Appendix A] for an introduction relevant to this paper. For $C^{*}$-algebras we write $\otimes$ for the spacial (minimal) tensor product, and for von Neumann algebras we write $\bar{\otimes}$ for the von Neumann algebra tensor product.

Given Banach spaces $E, F$ and a bounded linear map $T: E \rightarrow F^{*}$, we can consider the restriction of the Banach space adjoint, $\left.T^{*}\right|_{F}: F \rightarrow E^{*}$, and the adjoint of this, $\tilde{T}=\left(\left.T^{*}\right|_{F}\right)^{*}: E^{* *} \rightarrow F^{*}$ is the weak $k^{*}$-extension of $T$. If $\kappa_{E}: E \rightarrow E^{* *}$ is the canonical map, then $\tilde{T} \kappa_{E}=T$. When $E=A$ is a $C^{*}$-algebra and $F^{*}=M$ a von Neumann algebra, we speak of the normal extension.

A $C^{*}$-bialgebra if a pair $(A, \Delta)$ where $A$ is a $C^{*}$-algebra and $\Delta: A \rightarrow M(A \otimes A)$ a non-degenerate $*$-homomorphism with $(\Delta \otimes \mathrm{id}) \Delta=(\mathrm{id} \otimes \Delta) \Delta$. The examples of most interest to use are the locally compact quantum groups, [25, which, rather roughly, are $C^{*}$-bialgebras with invariant weights. For readable introductions see [22, 41]. We need really rather little of the theory, and so we shall just develop what we need as we come to it. We will however setup a little notation. We think of a quantum group as an abstract object $\mathbb{G}$, and then write $C_{0}(\mathbb{G})$ for the $C^{*}$-algebra arising. In general (because of amenability issues) there might be several natural $C^{*}$-algebras to consider. For example, all cocommutative locally compact quantum groups arise as $C_{r}^{*}(G)$, the reduced group $C^{*}$-algebra of a locally compact group $G$. However, here we could also consider the full, or universal, group $C^{*}$-algebra $C^{*}(G)$. Similarly, we can consider $C_{0}^{u}(\mathbb{G})$ the universal $C^{*}$-algebra representing $\mathbb{G}$, see $[23$; the main difference is that the weights on $C_{0}^{u}(\mathbb{G})$ might fail to be faithful. In this paper, we choose to work with $C_{0}^{u}(\mathbb{G})$, because it is the maximal choice, but the theory works the same if we use $C_{0}(\mathbb{G})$; compare with Remark 3.30 below.

The natural morphisms between $C^{*}$-bialgebras $\left(A, \Delta_{A}\right)$ and $\left(B, \Delta_{B}\right)$ are non-degenerate $*$-homomorphisms $\theta: B \rightarrow M(A)$ with $\Delta_{A} \circ \theta=(\theta \otimes \theta) \circ \Delta_{B}$. We "reverse the arrows" so that if $S, T$ are locally compact semigroups and $A=C_{0}(S), B=C_{0}(T)$ with $\Delta_{A}, \Delta_{B}$ the canonical choices, then we exactly recover the continuous semigroup homomorphisms $S \rightarrow T$. In the setting of locally compact quantum groups, the paper [30] shows that, so long as we work at the universal level with $C_{0}^{u}(\mathbb{G})$, this notion of morphism can be recast in a variety of other natural ways.

A unitary corepresentation of a $C^{*}$-bialgebra $(A, \Delta)$ on a Hilbert space $H$ is a unitary $U \in M(A \otimes$ $\left.\mathcal{B}_{0}(H)\right)$ with $(\Delta \otimes \mathrm{id})(U)=U_{13} U_{23}$. We sometimes write $H_{U}$ for $H$ to emphasise the link between $U$ and $H$. Given two corepresentations $U, V$ their tensor product is $U \oplus V=U_{12} V_{13} \in M\left(A \otimes \mathcal{B}_{0}\left(H_{U}\right) \otimes \mathcal{B}_{0}\left(H_{V}\right)\right)=$ $M\left(A \otimes \mathcal{B}_{0}\left(H_{U} \otimes H_{V}\right)\right)$.

\section{Semigroups}

A semitopological semigroup $S$ is a semigroup which is also a (Hausdorff) topological space, such that the product is separately continuous. Following [39], but with slightly different terminology, we shall say that a semitopological semigroup $S$ is $(\mathrm{WCH})$ if $S$ is homeomorphic, as a semigroup, to a sub-semigroup of contractions on a Hilbert space $H$, denoted $\mathcal{B}(H)_{\|\cdot\| \leq 1}$, with the weak*-topology. If we replace the weak*-topology by the strong*-topology, we obtain the property (SCH).

Let WCH be the collection of all WCH semigroups, made into a category with morphisms the continuous semigroup homomorphisms. Similarly define SCH. Both of these categories are full subcategories of STS, the category of all semitopological semigroups with continuous semigroup homomorphisms. Finally, let LCG be the category of locally compact groups with continuous group homomorphisms, so also LCG is a full subcategory of STS. Let $\mathrm{CSCH}, \mathrm{CWCH}, \mathrm{CSTS}, \mathrm{CG}$ be the full subcategories of compact semigroups in, respectively, SCH, WCH, STS and LCG.

In this main, first section, we wish to study WCH from a "noncommutative topology" viewpoint: that is, to find a class of $C^{*}$-algebras with extra structure such that the commutative algebras exactly correspond to members of $\mathrm{WCH}$, and such that noncommutative algebras still have interesting properties. 


\subsection{The function-free picture}

In this section, we shall show how to form natural algebras from compact semigroups of Hilbert space contractions. This lays the framework, in the following section, to describe such semigroups purely through certain generalised $\mathrm{C}^{*}$-bialgebras.

Let $S \in \mathrm{CWCH}$ or $\mathrm{CSCH}$, say $S \subseteq \mathcal{B}(H)_{\|\cdot\| \leq 1}$, equipped with either the relative weak* or SOT* topologies, as appropriate. Here we work with compact semigroups for convenience; see Section 3.5 below for some comments on the locally compact case. Set $A=C(S)$, and let $S C(S \times S)$ be the space of bounded separately continuous functions $S \times S \rightarrow \mathbb{C}$. Following [34, Section 2] we find that for $f \in S C(S \times S)$, integrating out one variable gives a $C(S)$ function, and so there is a well-defined map

$$
M(S) \times M(S) \rightarrow \mathbb{C} ; \quad(\mu, \lambda) \mapsto \int_{S} \int_{S} f(s, t) d \mu(s) d \lambda(t)
$$

This sets up an isometric isomorphism between $S C(S \times S)$ and $\mathcal{B}_{w^{*}}(M(S), M(S)$; $\mathbb{C})$ the space of bounded separately weak*-continuous bilinear maps $M(S) \times M(S) \rightarrow \mathbb{C}$, see [34, Proposition 2.5] (which makes use of [19, 21]).

The coproduct for $C(S)$ is naturally realised as a coassociative map $\Phi: C(S) \rightarrow S C(S \times S) ; \Phi(f)(s, t)=$ $f(s t)$ for $f \in C(S), s, t \in S$. Working in the category of Operator Spaces let $\widehat{\otimes}$ be the operator space projective tensor product. As $C(S)$ is commutative, $C(S)^{*}=M(S)$ has the maximal operator space structure, and so

$$
\mathcal{B}_{w^{*}}(M(S), M(S) ; \mathbb{C}) \subseteq(M(S) \widehat{\otimes} M(S))^{*}=C(S)^{* *} \bar{\otimes} C(S)^{* *} .
$$

Motivated by this, for an arbitrary $C^{*}$-algebra $A$ we define $\mathcal{C B}_{w^{*}}\left(A^{*}, A^{*} ; \mathbb{C}\right)$ to be the collection of $x \in$ $A^{* *} \bar{\otimes} A^{* *}$ such that for each $\mu \in A^{*}$, the maps

$$
A^{*} \rightarrow \mathbb{C} ; \quad \lambda \mapsto\langle x, \lambda \otimes \mu\rangle,\langle x, \mu \otimes \lambda\rangle
$$

are both weak*-continuous. This is equivalent to defining

$$
\mathcal{C B}_{w^{*}}\left(A^{*}, A^{*} ; \mathbb{C}\right)=\left\{x \in A^{* *} \bar{\otimes} A^{* *}:(\mu \otimes \mathrm{id})(x),(\operatorname{id} \otimes \mu)(x) \in A\left(\mu \in A^{*}\right)\right\} .
$$

There is a problem here in that for non-commutative $A$, there is no reason why this need be a subalgebra of $A^{* *} \bar{\otimes} A^{* *}$; a counter-example is given by taking $A=\mathcal{B}_{0}(H)$ for an infinite-dimensional $H$ and letting $x \in \mathcal{B}(H \otimes H)$ be the tensor swap map, compare Remark 3.26 below.

Nonetheless, we can view $\Phi$ as a $*$-homomorphism $A \rightarrow A^{* *} \bar{\otimes} A^{* *}$ taking values in the subspace $\mathcal{C B}_{w^{*}}\left(A^{*}, A^{*} ; \mathbb{C}\right)$. Again, working with operator spaces, we have that

$$
A^{* *} \bar{\otimes} \mathcal{B}(H)=\left(A^{*} \widehat{\otimes} \mathcal{B}(H)_{*}\right)^{*}=\mathcal{C} \mathcal{B}\left(A^{*}, \mathcal{B}(H)\right) .
$$

As $A=C(S)$ is commutative, $\mathcal{C B}\left(A^{*}, \mathcal{B}(H)\right)=\mathcal{B}\left(A^{*}, \mathcal{B}(H)\right)$ and so we can define $V \in A^{* *} \bar{\otimes} \mathcal{B}(H)$ by

$$
\left\langle V(\mu), \omega_{\xi, \eta}\right\rangle=\int_{S}(x \xi \mid \eta) d \mu(x) \quad\left(\mu \in A^{*}=M(S), \xi, \eta \in H\right) .
$$

As $S$ carries the weak* or $\mathrm{SOT}^{*}$ topology, the map $S \mapsto \mathbb{C} ; x \mapsto(x \xi \mid \eta)$ is continuous, and so the integral is defined. Notice that $\|V\| \leq 1$.

Lemma 3.1. If $S \in C S C H$ then $V \in M\left(A \otimes \mathcal{B}_{0}(H)\right)$, but this need not be true for $S \in C W C H$.

Proof. Consider $f \otimes \theta_{\xi, \eta} \in A \otimes \mathcal{B}_{0}(H)$. Then for $\mu \in M(S), \omega_{\alpha, \beta} \in \mathcal{B}(H)_{*}$,

$$
\left\langle V\left(f \otimes \theta_{\xi, \eta}\right), \mu \otimes \omega_{\alpha, \beta}\right\rangle=\left\langle V(f \mu), \omega_{\xi, \beta}\right\rangle(\alpha \mid \eta)=\int_{S}(x \xi \mid \beta)(\alpha \mid \eta) f(x) d \mu(x)=\int_{S}(F(x) \alpha \mid \beta) d \mu(x),
$$


if $F \in C\left(S, \mathcal{B}_{0}(H)\right)=A \otimes \mathcal{B}_{0}(H)$ is defined by $F(x)=f(x) \theta_{x \xi, \eta}$. Notice that $F$ is continuous because $S \rightarrow H, x \mapsto x \xi$ is continuous, $S$ having the SOT ${ }^{*}$-topology. Similarly $\left(f \otimes \theta_{\xi, \eta}\right) V=F^{\prime}$ where $F^{\prime}(x)=$ $f(x) \theta_{\xi, x^{*} \eta}$ is also continuous.

However, if $S \in \mathrm{CWCH}$, for example $S=\mathcal{B}(H)_{\|\cdot\| \leq 1}$, then there are nets $\left(x_{i}\right)$ in $S$ which are weak*-null but not SOT-null, say $\left(x_{i} \xi\right)$ is not norm null. With $f=1$, we find that $F\left(x_{i}\right)=\theta_{x_{i} \xi, \eta} \nrightarrow \rightarrow 0=F(0)$ and so $F$ is not continuous.

Remark 3.2. We cannot expect $V$ to be unitary. Indeed, from the calculations above, for $f \in C(S), \theta \in$ $\mathcal{B}_{0}(H), \omega_{\xi, \eta} \in \mathcal{B}(H)_{*}$ and $x \in S$, setting $\mu=\delta_{x} \in M(S)$, we find that

$$
\left\langle V\left(\mu \otimes \omega_{\xi, \eta}\right), f \otimes \theta\right\rangle=\left\langle V, \mu f \otimes \omega_{\xi, \theta^{*} \eta}\right\rangle=\int_{S}(f(y) \theta y \xi \mid \eta) d \mu(y)=(f(x) \theta x \xi \mid \eta),
$$

and this determines $V\left(\mu \otimes \omega_{\xi, \eta}\right) \in M(S) \widehat{\otimes} \mathcal{B}(H)_{*}$ as $C(S) \otimes \mathcal{B}_{0}(H)$ is weak ${ }^{*}$-dense in $C(S)^{* *} \bar{\otimes} \mathcal{B}(H)$. If $V\left(\mu \otimes \omega_{\xi, \eta}\right)$ is approximately $\sum_{i} \mu_{i} \otimes \omega_{i}$, then

$$
\begin{aligned}
\left\langle V^{*} V, \mu \otimes \omega_{\xi, \eta}\right\rangle & \approx \sum_{i}\left\langle V^{*}, \mu_{i} \otimes \omega_{i}\right\rangle=\sum_{i} \overline{\left\langle V, \mu_{i}^{*} \otimes \omega_{i}^{*}\right\rangle}=\sum_{i} \overline{\int_{S}\left\langle y, \omega_{i}^{*}\right\rangle d \mu_{i}^{*}(y)} \\
& =\sum_{i} \int_{S} \overline{\left\langle y, \omega_{i}^{*}\right\rangle} d \mu_{i}(y)=\sum_{i} \int_{S}\left\langle y^{*}, \omega_{i}\right\rangle d \mu_{i}(y) .
\end{aligned}
$$

Now, for all $f, \theta$ we have that

$$
\sum_{i} \int_{S}\left\langle f(y) \theta, \omega_{i}\right\rangle d \mu_{i}(y)=\sum_{i}\left\langle\mu_{i}, f\right\rangle\left\langle\theta, \omega_{i}\right\rangle \approx\left\langle V\left(\mu \otimes \omega_{\xi, \eta}\right), f \otimes \theta\right\rangle=(f(x) \theta x \xi \mid \eta)
$$

and so by taking limits,

$$
\left\langle V^{*} V, \mu \otimes \omega_{\xi, \eta}\right\rangle=\left(x^{*} x \xi \mid \eta\right) .
$$

By contrast, $\left\langle 1, \mu \otimes \omega_{\xi, \eta}\right\rangle=\int_{S}(\xi \mid \eta) d \mu(y)=(\xi \mid \eta)$ and so $V^{*} V=1$ if and only if $x \in S \Longrightarrow x^{*} x=1$, that is, $S$ is a semigroup of isometries. Similarly, $V V^{*}=1$ only when $S$ is a semigroup of coisometries.

Lemma 3.3. For any $\omega \in \mathcal{B}(H)_{*}$ we have that $(\mathrm{id} \otimes \omega)(V) \in A=C(S)$ and the collection $\{(\mathrm{id} \otimes \omega)(V)$ : $\left.\omega \in \mathcal{B}(H)_{*}\right\}$ separates the points of $S$.

Proof. By the definition of $V$ we find that

$$
\langle\mu,(\mathrm{id} \otimes \omega)(V)\rangle=\int_{S}\langle x, \omega\rangle d \mu(x) \quad(\mu \in M(S)),
$$

and so $($ id $\otimes \omega)(V)$ is the continuous function $S \rightarrow \mathbb{C} ; x \mapsto\langle x, \omega\rangle$, a member of $A$. This collection will clearly separate points as $\omega \in \mathcal{B}(H)_{*}$ varies.

In general, there is no reason why $\left\{(\mathrm{id} \otimes \omega)(V): \omega \in \mathcal{B}(H)_{*}\right\}$ should be a sub-algebra of $C(S)$. However, by modifying the Hilbert space, we can obtain this. Let

$$
F(H)=\bigoplus_{m, n \in \mathbb{N}_{0}, m+n \geq 1} H^{m \otimes} \otimes \bar{H}^{n \otimes}
$$

and define an isometry $F: \mathcal{B}(H)_{\|\cdot\| \leq 1} \rightarrow \mathcal{B}(F(H))_{\|\cdot\| \leq 1}$ by

$$
F(x)=\bigoplus_{m, n \in \mathbb{N}_{0}, m+n \geq 1} x^{m \otimes} \otimes \bar{x}^{n \otimes} .
$$


A tedious calculation shows that $F$ is weak ${ }^{*}$-continuous and $\mathrm{SOT}^{*}$-continuous. Indeed, we can exploit boundedness and linearity to reduce the task to showing that $\mathcal{B}(H)_{\|\cdot\| \leq 1} \rightarrow \mathcal{B}\left(H^{m \otimes} \otimes \bar{H}^{n \otimes}\right)_{\|\cdot\| \leq 1}$ is weak* SOT $\left.^{*}\right)$-continuous, which is clear. As $S$ is compact, $F(S)$ is compact and $F$ restricts to a homeomorphism $F: S \rightarrow F(S)$. Compare this with the discussion in [39, Section 1.3] and [40, Section 4].

A further complication is that if $0 \in S$, then functions of the form (id $\otimes \omega)(V)$ can never "strongly separate" the points of $S$. However, let $H^{\prime}$ be an auxiliary Hilbert space (for example, $H^{\prime}=\mathbb{C}$ ) and define $S^{\prime}=\left\{x \oplus 1_{H^{\prime}}: x \in S\right\} \subseteq \mathcal{B}\left(H \oplus H^{\prime}\right)$. Then $S \rightarrow S^{\prime}, x \mapsto x \oplus 1_{H^{\prime}}$ is a semigroup homomorphism, and a homeomorphism. So we may always assume that $0 \notin S$.

Lemma 3.4. Form $V^{\prime}$ from $F(S)$ as above. Then the *-algebra generated by $A_{V}=\{(\operatorname{id} \otimes \omega)(V): \omega \in$ $\left.\mathcal{B}(H)_{*}\right\}$ is dense in $A_{V^{\prime}}=\left\{(\mathrm{id} \otimes \omega)\left(V^{\prime}\right): \omega \in \mathcal{B}(F(H))_{*}\right\}$. Under the assumption that $0 \notin S$, $A_{V^{\prime}}$ is a dense *-subalgebra of $C(S)$.

Proof. We find that coefficients of $H^{m \otimes} \otimes \bar{H}^{n \otimes}$ give adjoints of coefficients of $H^{n \otimes} \otimes \bar{H}^{m \otimes}$ and products of coefficients from $H^{m \otimes} \otimes \bar{H}^{n \otimes}$ and $H^{m^{\prime} \otimes} \otimes \bar{H}^{n^{\prime} \otimes}$ give coefficients of $H^{\left(m+m^{\prime}\right) \otimes} \otimes \bar{H}^{\left(n+n^{\prime}\right) \otimes}$. Thus $A_{V^{\prime}}$ is a *-subalgebra of $C(S)$. It's also clear that the *-algebra generated by $A_{V}$ will be dense in $A_{V^{\prime}}$.

By Stone-Weierstrass, $A_{V^{\prime}}$ will be dense in $C(S)$ if $A_{V^{\prime}}$ "strongly separates points", 9, Corollary 8.3, Chapter V], that is, if for each $x \in S$ there is $f \in A_{V^{\prime}}$ with $f(x) \neq 0$. But this is clear once we ensure that $0 \notin S$.

We finally establish some further properties of $(A, \Phi, V)$. For $\mu, \lambda \in A^{*}$ define $\langle\mu \star \lambda, a\rangle=\langle\Phi(a), \mu \otimes \lambda\rangle$ for $a \in A$.

Lemma 3.5. The bilinear map $\star$ turns $A^{*}$ into a Banach algebra, and the map $A^{*} \rightarrow \mathcal{B}(H) ; \mu \mapsto$ $(\mu \otimes \mathrm{id})(V)$ becomes a homomorphism for this product.

Proof. By construction,

$$
\langle\mu \star \lambda, a\rangle=\int_{S} \int_{S} a(x y) d \mu(x) d \lambda(y),
$$

and so, by using the version of Fubini's Theorem which holds here, compare [34, Lemma 2.4], the associativity of the product on $S$ gives that $\star$ is associative. Then for $\omega \in \mathcal{B}(H)_{*}$,

$$
\langle(\mu \star \lambda \otimes \mathrm{id})(V), \omega\rangle=\int_{S} \int_{S}(\mathrm{id} \otimes \omega)(V)(x y) d \mu(x) d \lambda(y)=\int_{S} \int_{S}\langle x y, \omega\rangle d \mu(x) d \lambda(y),
$$

while

$$
\langle(\mu \otimes \mathrm{id})(V)(\lambda \otimes \mathrm{id})(V), \omega\rangle=\int_{S}\langle x(\lambda \otimes \mathrm{id})(V), \omega\rangle d \mu(x)=\int_{S} \int_{S}\langle x y, \omega\rangle d \lambda(y) d \mu(x),
$$

so by another application of Fubini, it follows that $(\mu \star \lambda \otimes \mathrm{id})(V)=(\mu \otimes \mathrm{id})(V)(\lambda \otimes \mathrm{id})(V)$ as required.

\subsection{The non-commutative framework}

As promised, we can now formulate a certain class of generalised $\mathrm{C}^{*}$-bialgebras which, in the commutative case, completely capture the classes $\mathrm{CSCH}$ and $\mathrm{CWCH}$. We start with a definition, and then justify that this is sensible.

Definition 3.6. $A C^{*}$-Eberlein algebra is a quadruple $\mathbb{S}=(A, \Phi, V, H)$ where:

1. $A$ is a $C^{*}$-algebra, $\Phi: A \rightarrow A^{* *} \bar{\otimes} A^{* *}$ is a unital $*$-homomorphism and $V \in A^{* *} \bar{\otimes} \mathcal{B}(H)$ is a contraction;

2. $A_{V}:=\left\{(\iota \otimes \omega)(V): \omega \in \mathcal{B}(H)_{*}\right\}$ is a subset of $A \subseteq A^{* *}$, and is norm dense in $A$; 
3. The bilinear product on $A^{*}$ given by $\langle\mu \star \nu, a\rangle=\langle\Phi(a), \mu \otimes \nu\rangle$ is associative;

4. $V$ and $\Phi$ are connected in the sense that the map $A^{*} \rightarrow \mathcal{B}(H) ; \mu \mapsto(\mu \otimes \iota)(V)$ is a homomorphism.

Recall that if $A$ is not unital, then by $\Phi$ being unital we mean, for example, that the normal extension $\tilde{\Phi}: A^{* *} \rightarrow A^{* *} \bar{\otimes} A^{* *}$ is unital (or equivalently that for any bounded approximate identity $\left(e_{\alpha}\right)$ of $A$ the net $\left(\Phi\left(e_{\alpha}\right)\right)$ converges weak ${ }^{*}$ to $\left.1 \in A^{* *} \bar{\otimes} A^{* *}\right)$.

Remark 3.7. Axiom (41) is exactly the statement that $V$ is a "corepresentation", here interpreted to mean that

$$
\Phi((\mathrm{id} \otimes \omega)(V))=(\mathrm{id} \otimes \mathrm{id} \otimes \omega)\left(V_{13} V_{23}\right) \quad\left(\omega \in \mathcal{B}(H)_{*}\right) .
$$

This makes sense, as we can form $V_{13} V_{23}$ in $A^{* *} \bar{\otimes} A^{* *} \bar{\otimes} \mathcal{B}(H)$. Alternatively, if we let $\tilde{\Phi}: A^{* *} \rightarrow A^{* *} \bar{\otimes} A^{* *}$ be the normal extension of $\Phi$, then this says precisely that $(\tilde{\Phi} \otimes \mathrm{id})(V)=V_{13} V_{23}$.

We also have that $A$ and $V$ uniquely determine $\Phi$. Indeed, if $\Phi^{\prime}$ is another coproduct satisfying (3) and (4) then

$$
\Phi^{\prime}((\mathrm{id} \otimes \omega)(V))=(\mathrm{id} \otimes \mathrm{id} \otimes \omega)\left(V_{13} V_{23}\right)=\Phi((\mathrm{id} \otimes \omega)(V)),
$$

for all $\omega \in \mathcal{B}(H)_{*}$. By (2) and continuity, it then follows that $\Phi^{\prime}=\Phi$

\subsection{Links with the Haagerup tensor product}

In Section 3.1 we carefully introduced to space $\mathcal{C B}_{w^{*}}\left(A^{*}, A^{*} ; \mathbb{C}\right)$ but in our axioms have made no mention of it. By using the Haagerup tensor product, studied in this short section, we can show that actually automatically $\Phi$ takes values in $\mathcal{C B}_{w^{*}}\left(A^{*}, A^{*} ; \mathbb{C}\right)$.

Let $A, B$ be $C^{*}$-algebras. The extended (or weak*) Haagerup tensor product of $A$ with $B$ is the collection of $x \in A^{* *} \bar{\otimes} B^{* *}$ with

$$
x=\sum_{i} x_{i} \otimes y_{i}
$$

with $\sigma$-weak convergence, for some families $\left(x_{i}\right)$ in $A$ and $\left(y_{i}\right)$ in $B$, such that $\sum_{i} x_{i} x_{i}^{*}$ and $\sum_{i} y_{i}^{*} y_{i}$ converge $\sigma$-weakly in $A^{* *}$ and $B^{* *}$ respectively. The natural norm is then

$$
\|x\|_{e h}=\inf \left\{\left\|\sum_{i} x_{i} x_{i}^{*}\right\|^{1 / 2}\left\|\sum_{i} y_{i}^{*} y_{i}\right\|^{1 / 2}: x=\sum_{i} x_{i} \otimes y_{i}\right\}
$$

and we write $A \otimes^{e h} B$ for the resulting normed space. See [6] or [16] for further details.

Continuing with the notation of the previous section, let $\left(e_{i}\right)$ be an orthogonal basis for $H$, so that for $\mu, \mu^{\prime} \in A^{*}$ and $\xi, \eta \in H$,

$$
\begin{aligned}
=\left((\mu \otimes \iota)(V)\left(\mu^{\prime} \otimes \iota\right)(V) \xi \mid \eta\right) & =\sum_{i}\left((\mu \otimes \mathrm{id})(V) e_{i} \mid \eta\right)\left(\left(\mu^{\prime} \otimes \mathrm{id}\right)(V) \xi \mid e_{i}\right) \\
& =\sum_{i}\left\langle\mu \otimes \mu^{\prime},\left(\operatorname{id} \otimes \omega_{e_{i}, \eta}\right)(V) \otimes\left(\mathrm{id} \otimes \omega_{\xi, e_{i}}\right)(V)\right\rangle .
\end{aligned}
$$

Now, the sum

$$
\sum_{i}\left(\mathrm{id} \otimes \omega_{e_{i}, \eta}\right)(V) \otimes\left(\mathrm{id} \otimes \omega_{\xi, e_{i}}\right)(V)
$$

converges in the extended Haagerup tensor product $A \otimes^{e h} A$. Furthermore, by conditions (2), (3) and (44), this is equal to

$$
\left\langle\Phi\left(\left(\iota \otimes \omega_{\xi, \eta}\right)(V)\right), \mu \otimes \mu^{\prime}\right\rangle
$$

and so we conclude that

$$
\Phi\left(\left(\iota \otimes \omega_{\xi, \eta}\right)(V)\right)=\sum_{i}\left(\mathrm{id} \otimes \omega_{e_{i}, \eta}\right)(V) \otimes\left(\mathrm{id} \otimes \omega_{\xi, e_{i}}\right)(V) .
$$

In particular, this shows that $\Phi$ does indeed take values in $\mathcal{C B}_{w^{*}}\left(A^{*}, A^{*} ; \mathbb{C}\right)$. 
Remark 3.8. We would really like to understand the notion of "separately continuous" in the noncommutative setting; in the previous section we used [34] to setup an isomorphism between $S C(S \times S)$ and $\mathcal{C B}_{w^{*}}\left(A^{*}, A^{*} ; \mathbb{C}\right)$. Indeed, it would be tempting to believe that maybe we could use the extended Haagerup tensor product in place of $\mathcal{C B}_{w^{*}}\left(A^{*}, A^{*} ; \mathbb{C}\right)$. This appears to be too general a notion however; see Remark 8.1 below.

\subsection{Commutative $C^{*}$-Eberlein algebras are classical semigroups}

Theorem 3.9. For any $S \in C W C H$ or $S \in C S C H$, with $0 \notin S$, we can canonically construct a $C^{*}$ Eberlein algebra $S=\left(C(S), \Phi_{S}, V_{S}, H_{S}\right)$. Conversely, if $\mathbb{S}=(A, \Phi, V, H)$ with $A$ unital and commutative, then there is $S \in C W C H$ with $\mathbb{S}=S$ and $0 \notin S$. Furthermore, we have that $V_{S} \in M\left(A \otimes \mathcal{B}_{0}(H)\right)$ if and only if $S \in S C H$.

Proof. The first claim follows immediately from the construction in Section 3.1. Conversely, let $(A, \Phi, V, H)$ be a $C^{*}$-Eberlein algebra with $A=C(S)$ for some compact Hausdorff space $S$. By the previous section, we know that

$$
\Phi: C(S) \rightarrow \mathcal{B}_{w^{*}}(M(S), M(S) ; \mathbb{C})=S C(S \times S) .
$$

So we get a product on $S$ by

$$
f(s t)=\left\langle\Phi(f), \delta_{s} \otimes \delta_{t}\right\rangle \quad(s, t \in S, f \in C(S)),
$$

and for all $f \in C(S)$ the map $(s, t) \mapsto f(s t)$ is separately continuous. So by condition (3), $S$ is a semitopological semigroup and $\Phi=\Phi_{S}$.

Define $\pi: S \rightarrow \mathcal{B}(H)_{\|\cdot\| \leq 1}$ by $\pi(s)=\left(\delta_{s} \otimes \iota\right)(V)$. By (4) we see that $\pi$ is a homomorphism, and from (22) it follows that $\pi$ is injective. Furthermore, if $s_{\alpha} \rightarrow s$ in $S$, then by (2),

$$
\lim _{\alpha}\left\langle\pi\left(s_{\alpha}\right), \omega\right\rangle=\lim _{\alpha}\left\langle\delta_{s_{\alpha}},(\operatorname{id} \otimes \omega)(V)\right\rangle=\left\langle\delta_{s},(\operatorname{id} \otimes \omega)(V)\right\rangle=\langle\pi(s), \omega\rangle . \quad\left(\omega \in \mathcal{B}(H)_{*}\right) .
$$

So $\pi$ is continuous if we give $\mathcal{B}(H)_{\|\cdot\| \leq 1}$ the relative weak*-topology. As $S$ is compact it follows that $\pi: S \rightarrow \pi(S)$ is a homeomorphism, if we give $\pi(S)$ the relative weak*-topology. We henceforth identify $S$ and $\pi(S)$. It follows that

$$
\langle V, \mu \otimes \omega\rangle=\langle\mu,(\mathrm{id} \otimes \omega)(V)\rangle=\int_{S}(\mathrm{id} \otimes \omega)(V)(x) d \mu(x)=\int_{S}\langle\pi(x), \omega\rangle d \mu(x),
$$

and so $V=V_{S}$ the canonical corepresentation. Condition (2) ensures that $0 \notin S$.

If $S \in \mathrm{SCH}$ then $V_{S} \in M\left(A \otimes \mathcal{B}_{0}(H)\right)$. Conversely, if $V_{S} \in M\left(A \otimes \mathcal{B}_{0}(H)\right) \cong C_{S O T^{*}}^{b}(S, \mathcal{B}(H))$ then as $V_{S}$ is the inclusion map $S \rightarrow \mathcal{B}(H)_{\|\cdot\| \leq 1}$, it follows that $(S$, weak $) \rightarrow\left(\mathcal{B}(H)_{\|\cdot\| \leq 1}, S O T^{*}\right)$ is continuous. So $S$ is SOT* compact, hence closed, and $S \rightarrow\left(\mathcal{B}(H)_{\|\cdot\| \leq 1}, S O T^{*}\right)$ is a homeomorphism onto its range, as required.

Let us call $V$ the "generating corepresentation" for $\mathbb{S}$. We note that $V$ is not unique (the above proof suggests that if $\mathbb{S}$ is classical, $A=C(S)$ for $S \in \mathrm{CWCH}$, then $V$ is always the "canonical" $V_{S}$; but this is only after we have identified $S$ with $\pi(S)$, where $\pi$ is given by $V$ ). Indeed, if $u: H \rightarrow K$ is a unitary, then $\left(1 \otimes u^{*}\right) V(1 \otimes u)$ will also generate $(A, \Phi)$. Slightly less trivially, if $U \in A^{* *} \bar{\otimes} \mathcal{B}(K)$ is such that $($ id $\otimes \omega)(U) \in A$ for all $\omega \in \mathcal{B}(K)_{*}$ and $\Phi((\operatorname{id} \otimes \omega)(U))=($ id $\otimes$ id $\otimes \omega)\left(U_{13} U_{23}\right)$, then $U \oplus V$ will also be generating. In particular, we can take direct sums of $V$ with itself.

Remark 3.10. Our philosophy will be that $(A, \Phi)$ is the primary object, and we assume the existence of some $V$. Axiom (2) just says that $V$ is "sufficiently large" in some sense. 
Remark 3.11. We can make use of this to weaken (2). If all we have is that (id $\otimes \omega)(V) \in A$ for all $\omega$, then let $U=V \oplus V_{12} V_{13} \oplus V_{12} V_{13} V_{14} \oplus \cdots$ on $H \oplus H \otimes H \oplus H^{\otimes 3} \oplus \cdots$. As $\left(\right.$ id $\left.\otimes \omega \otimes \omega^{\prime}\right)\left(V_{12} V_{13}\right)=$ (id $\otimes \omega)(V)\left(\right.$ id $\left.\otimes \omega^{\prime}\right)(V)$, and so forth, it follows that $A_{U}$ will be the subalgebra of $A$ generated by $A_{V}$. As $\Phi$ is a homomorphism, it's easy to see that $V$ will also be a corepresentation. Thus, it is enough that there exists $V$ with $($ id $\otimes \omega)(V) \in A$ for all $\omega$ and such that the closure of $A_{V}$ is $*$-closed- once we have this, we can form $U$ and get $A_{U}$ to be a *-algebra, which we then require to be dense.

\subsection{The locally compact case}

Let $S$ be a merely locally compact, semitopological semigroup. Then translates need not leave $C_{0}(S)$ invariant: for example, if $0 \in S$ is a distinguished point and $S$ has the "zero multiplication", st $=0$ for all $s, t \in S$, then if $S$ is non-compact, for any $t \in S, f(t s)=f(s t)=f(0)$ for all $s$, so $f(t \cdot)=f(\cdot t)=f(0) 1 \notin$ $C_{0}(S)$ whenever $f(0) \neq 0$.

If we want translations to act on $C_{0}(S)$ then we need left and right translations to be proper maps (inverse image of compact is compact). Equivalently, if $\left(s_{\alpha}\right)$ is a net in $S$ tending to infinity (meaning that for all compact $K \subseteq S$ there is $\alpha_{0}$ so that $s_{\alpha} \notin K$ for $\left.\alpha \leq \alpha_{0}\right)$ then also $\left(t s_{\alpha}\right)$ and $\left(s_{\alpha} t\right)$ converge to $\infty$. Furthermore, once we have this condition, it is not hard to check that the one-point compactification, $S_{\infty}$, becomes semitopological for the product $\infty s=s \infty=\infty$ for all $s \in S$.

Proposition 3.12. Let $(A, \Phi, V, H)$ be a $C^{*}$-Eberlein algebra with $A$ commutative and non-unital. There is a locally compact, non-compact, $S \in W C H$ with $A=C_{0}(S)$ and $\Phi, V, H$ being the canonical choices. Furthermore, the one-point compactification $S_{\infty}$ is canonically a semitopological semigroup with $S_{\infty} \in$ CWCH.

Proof. We could follow the proof of Theorem 3.9, using [34] to again identify $S C_{0}(S \times S)$. However, it is not now clear that $\pi: S \rightarrow \mathcal{B}(H)_{\|\cdot\| \leq 1}$ will be a homeomorphism, as $S$ is not compact.

However, notice that axiom (22) tells us that the coefficients $s \mapsto\langle\pi(s), \omega\rangle$ are in $C_{0}(S)$ for any $\omega \in \mathcal{B}(H)_{*}$. So if $s_{\alpha} \rightarrow \infty$ then $\left\langle\pi\left(s s_{\alpha}\right), \omega\right\rangle=\left\langle\pi\left(s_{\alpha}\right), \omega \pi(s)\right\rangle \rightarrow 0$ and similarly $\left\langle\pi\left(s_{\alpha} s\right), \omega\right\rangle \rightarrow 0$. As such functions are norm-dense in $C_{0}(S)$ this shows that $f\left(s s_{\alpha}\right) \rightarrow 0, f\left(s_{\alpha} s\right) \rightarrow 0$ for all $s \in S, f \in C_{0}(S)$. It follows that translates in $S$ are proper maps, and so we can turn $S_{\infty}$ into a semitopological semigroup in the canonical way.

Furthermore, this argument also shows that $s_{\alpha} \rightarrow \infty$ in $S$ implies that $\pi\left(s_{\alpha}\right) \rightarrow 0$ weak* in $\mathcal{B}(H)$. Hence $\pi_{\infty}: S_{\infty} \rightarrow \mathcal{B}(H)_{\|\cdot\| \leq 1} ; S \ni s \mapsto \pi(s), \infty \mapsto 0$ defines an injective, continuous semigroup homomorphism. As $S_{\infty}$ is compact, this is a homeomorphism. Once again, this gives $0 \in \pi_{\infty}\left(S_{\infty}\right)$, but we can follow the construction before Lemma 3.4 and replace $H$ by $H \oplus \mathbb{C}$ and replace 0 by $0 \oplus 1_{\mathbb{C}}$.

Now suppose that $\left(s_{\alpha}\right)$ is a net in $S$ with $\pi\left(s_{\alpha}\right) \rightarrow \pi(s)$ for some $s \in S$. Then $\pi_{\infty}\left(s_{\alpha}\right) \rightarrow \pi_{\infty}(s)$ and so $s_{\alpha} \rightarrow s$ in $S_{\infty}$, and so $s_{\alpha} \rightarrow s$ in $S$. Thus $\pi$ is a homeomorphism, and so we can indeed follow the rest of the proof of Theorem 3.9 .

Remark 3.13. In fact, there is an analogous construction in the non-commutative setting, which we can view as a way to "compactify" in a minimal sense; see Section 5 for further details. Indeed, given $(A, \Phi, V, H)$ with $A$ not unital, consider $B=A \oplus \mathbb{C} 1$ the unitisation, made into a $C^{*}$-algebra in the usual way (e.g. consider $B \subseteq M(A)$ to obtain a $C^{*}$-norm). So $A$ is a co-dimension one ideal in $B$, and hence $B^{* *}=A^{* *} \oplus 1_{B}$. One concrete way to see this is to let $A \subseteq \mathcal{B}(K)$ be the universal representation, so $A^{* *} \cong A^{\prime \prime} \subseteq \mathcal{B}(K)$. Then let $A$ act on $\mathcal{B}(K \oplus \mathbb{C})$ as $a(\xi \oplus t)=a \xi \oplus 0$ for $a \in A, \xi \in K, t \in \mathbb{C}$, and let $1_{B}$ act as the identity on $K \oplus \mathbb{C}$. Thus we obtain a $*$-representation of $B$, and under $B^{* *} \cong A^{* *} \oplus \mathbb{C} 1_{B}$, we see that $B^{* *}$ acts on $K \oplus \mathbb{C}$ in exactly the same way.

Define $\Phi_{B}$ in the obvious way (so $\Phi_{B}(1)=1 \otimes 1$ ). Let $H_{B}=H \oplus \mathbb{C}$, so we may identify $A^{* *} \bar{\otimes} \mathcal{B}(H)$ and $1_{B} \bar{\otimes} \mathbb{C}$ as subalgebras of $B^{* *} \bar{\otimes} \mathcal{B}\left(H_{B}\right)$. Under this, let $V_{B}=V \oplus 1_{B} \otimes 1_{\mathbb{C}}$, so $V_{B}$ is a contraction. So axioms (11) and (3) hold. Then slices of $V_{B}$ give us slices of $V$ and scalar multiples of $1_{B}$, from which axiom (22) follows. As $B^{*} \cong A^{*} \oplus \mathbb{C}$, it is easy to verify axiom (44). 
We remark that $V_{B}$ is never unitary (even if $V$ is) and that $V_{B}$ might fail to be a multiplier of $B \otimes \mathcal{B}_{0}\left(H_{B}\right)$ even if $V \in M\left(A \otimes \mathcal{B}_{0}(H)\right)$ (performing the calculation, you end up needing that also $V(1 \otimes \theta) \in B \otimes \mathcal{B}_{0}(H)$ for all $\left.\theta \in \mathcal{B}_{0}(H)\right)$.

Then, if $A=C_{0}(S)$, then this abstract construction is exactly the same as forming $S_{\infty}$ as in the previous proof, followed by the procedure to ensure $0 \notin S_{\infty}$. (A little care is required in this calculation, as while $C\left(S_{\infty}\right) \cong C_{0}(S) \oplus \mathbb{C} 1$ and hence $C\left(S_{\infty}\right)^{*} \cong M(S) \oplus \mathbb{C}$ this splitting is not naively as $M(S) \oplus \mathbb{C} \delta_{\infty}$.)

Notice that even if $S \in \mathrm{SCH}$, it's unlikely that $S_{\infty} \in \mathrm{CSCH}$; indeed, we'd need that $s_{\alpha} \rightarrow \infty$ in $S$ implied that $s_{\alpha} \rightarrow 0$ in the $\mathrm{SOT}^{*}$.

Thus, in the commutative setting, $C^{*}$-Eberlein algebras give precisely compact $S \in \mathrm{WCH}$ or $S \in \mathrm{SCH}$, or give locally compact $S$ such that $S_{\infty}$ becomes semitopological. (Equivalently, those locally compact semigroups obtained by removing a "semigroup zero" from a member of $\mathrm{CWCH}$ ).

\subsection{Morphisms}

Having now defined the objects we wish to study, we now look at making these objects into a category; our motivation here is to eventually build a "compactifcation" theory.

Definition 3.14. For $i=1,2$ let $\mathbb{S}_{i}=\left(A_{i}, \Phi_{i}, V_{i}, H_{i}\right)$ be $C^{*}$-Eberlein algebras. A morphism $\mathbb{S}_{1} \rightarrow \mathbb{S}_{2}$ is a non-degenerate $*$-homomorphism $\theta: A_{2} \rightarrow M\left(A_{1}\right)$ which "intertwines the coproducts", in the weak sense that $\theta^{*}: A_{1}^{*} \rightarrow A_{2}^{*}$ is a homomorphism.

Lemma 3.15. With $\mathbb{S}_{1}, \mathbb{S}_{2}$ as above, and $\theta: A_{2} \rightarrow M\left(A_{1}\right)$ a non-degenerate $*$-homomorphism, the following are equivalent:

1. $\theta$ is a morphism;

2. regard $M\left(A_{1}\right) \subseteq A_{1}^{* *}$ and hence $\theta: A_{2} \rightarrow A_{1}^{* *}$ is a homomorphism; let $\tilde{\theta}: A_{2}^{* *} \rightarrow A_{1}^{* *}$ be the normal extension. Similarly let $\tilde{\Phi}_{1}: A_{1}^{* *} \rightarrow A_{1}^{* *} \bar{\otimes} A_{1}^{* *}$ be the normal extension. Then we have that $\tilde{\Phi}_{1} \circ \theta=(\tilde{\theta} \otimes \tilde{\theta}) \circ \Phi_{2} ;$

3. the map $A_{1}^{*} \rightarrow \mathcal{B}\left(H_{2}\right) ; \mu \mapsto(\mu \circ \tilde{\theta} \otimes \mathrm{id})\left(V_{2}\right)$ is a homomorphism;

Proof. That (11) and (2) are equivalent is routine. That (11) and (3) are equivalent follows by using axiom (21) and Remark 3.7.

Suppose that $\theta: A_{2} \rightarrow A_{1}$ is a surjection. Then $U=(\tilde{\theta} \otimes \mathrm{id})\left(V_{2}\right)$ is a generator for $A_{1}$ : indeed, all that remains to check is that $A_{V}=\left\{(\mathrm{id} \otimes \omega)(V): \omega \in \mathcal{B}\left(H_{2}\right)_{*}\right\}=\left\{\theta(\mathrm{id} \otimes \omega)\left(V_{2}\right): \omega \in \mathcal{B}\left(H_{2}\right)_{*}\right\}=\theta\left(A_{V_{2}}\right)$ is dense in $A_{1}$; but this is clear.

Definition 3.16. Let $\mathbb{G}$ be a locally compact quantum group, and let $\mathbb{S}=(A, \Phi, V, H)$ be a $C^{*}$-Eberlein algebra. A morphism $\mathbb{G} \rightarrow \mathbb{S}$ is a non-degenerate $*$-homomorphism $\theta: A \rightarrow M\left(C_{0}^{u}(\mathbb{G})\right)$ such that:

1. $\theta^{*}: C_{0}^{u}(\mathbb{G})^{*} \rightarrow A^{*}$ is a homomorphism of Banach algebras;

2. we require that $U=(\tilde{\theta} \otimes \mathrm{id})(V) \in C_{0}^{u}(\mathbb{G})^{* *} \bar{\otimes} \mathcal{B}(H)$ be a "possibly degenerate unitary corepresentation". This means that $\left(\tilde{\Delta}_{u} \otimes \mathrm{id}\right)(U)=U_{13} U_{23}$, and there is an orthogonal projection $p \in \mathcal{B}(H)$ with $U^{*} U=U U^{*}=1 \otimes p$.

Let us motivate this definition from the commutative situation, and then state some clarifying lemmas.

Proposition 3.17. Let $\mathbb{G}=G$ a locally compact group, and $\mathbb{S}=S$ a classical semigroup (so $S \in C W C H$ or CSCH). Let $S$ be presented as acting on the Hilbert space $H$. There is a bijection between morphisms $\mathbb{G} \rightarrow$ $\mathbb{S}$ and representations $\pi: G \rightarrow \mathcal{B}(H)$ with a closed subspace $H_{0} \subseteq H$, such that, under the decomposition $H=H_{0} \oplus H_{0}^{\perp}, \pi$ acts as unitaries on $H_{0}$, and as zero on $H_{0}^{\perp}$. In particular, condition (2) above is automatic in this case. 
Proof. Given a morphism $\theta: C(S) \rightarrow M\left(C_{0}(G)\right)$, there is a unique continuous map $\phi: G \rightarrow S$ with $\theta(f)=f \circ \phi$ for each $f \in C(S)$. Condition (1) implies that $\phi$ is a homomorphism. Let $\iota: S \rightarrow \mathcal{B}(H)_{\|\cdot\| \leq 1}$ be the inclusion, and let $\pi=\iota \circ \phi$ a group representation of $G$ by contractions. Thus $p=\pi(e)$ is a contractive idempotent, hence an orthogonal projection, on $H$; let $H_{0}=p(H)$. Then, for any $g \in G$, we see that $\pi(g)=\pi(e g)=p \pi(g)=\pi(g e)=\pi(g) p=p \pi(g) p$ and so $H_{0}$ is invariant for $\pi$. Furthermore, if $\xi \in H_{0}^{\perp}$ then $\pi(g) \xi=\pi(g) p \xi=0$, and so $\pi=0$ on $H_{0}^{\perp}$. As $S \in \mathrm{WCH}$, and $\phi$ is continuous, we see that $\pi$ is weakly, hence also strongly, continuous.

As $V$ "is" the map $\iota$, it follows that $U=(\tilde{\theta} \otimes \mathrm{id})(V) \in C_{0}(G)^{* *} \bar{\otimes} \mathcal{B}(H)$ is the canonical corepresentation associated to $\pi$. Let us be a little more careful: for $\omega=\omega_{\xi, \eta} \in \mathcal{B}(H)_{*}$, we find that

$$
(\operatorname{id} \otimes \omega)(U)=\tilde{\theta}((\operatorname{id} \otimes \omega)(V))=\tilde{\theta}\left((\pi(g) \xi \mid \eta)_{g \in G}\right) \in C^{b}(G)=M\left(C_{0}(G)\right) \subseteq C_{0}(G)^{* *},
$$

from which the claim follows. As $\pi$ is continuous, it follows that really $U$ is a member of $M\left(C_{0}(G) \otimes\right.$ $\mathcal{B}(H)) \subseteq C_{0}(G)^{* *} \bar{\otimes} \mathcal{B}(H)$. It readily follows that $U^{*} U=U U^{*}=1 \otimes p$ as claimed.

Clearly we can also reverse this process.

Lemma 3.18. Let $\mathbb{G}, \mathbb{S}$ be as above, and let $\theta: A \rightarrow M\left(C_{0}^{u}(\mathbb{G})\right)$ be a non-degenerate *-homomorphism. Then the following are equivalent:

1. $\theta^{*}: C_{0}^{u}(\mathbb{G})^{*} \rightarrow A^{*}$ is a homomorphism;

2. if $\tilde{\theta}: A^{* *} \rightarrow C_{0}^{u}(\mathbb{G})^{* *}$ is the normal extension, then $\left.\tilde{\Delta}_{u} \circ \tilde{\theta}\right|_{A}=(\tilde{\theta} \otimes \tilde{\theta}) \circ \Phi$.

3. $C_{0}^{u}(\mathbb{G})^{*} \rightarrow \mathcal{B}(H) ; \mu \mapsto(\mu \circ \tilde{\theta} \otimes \mathrm{id})(V)$ is a homomorphism.

Lemma 3.19. If $U \in C_{0}^{u}(\mathbb{G})^{* *} \bar{\otimes} \mathcal{B}(H)$ with $U^{*} U=U U^{*}=1 \otimes p$ for a projection $p \in \mathcal{B}(H)$, we have that $U \in M\left(C_{0}^{u}(\mathbb{G}) \otimes \mathcal{B}_{0}(H)\right)$.

Proof. We follow [23]. Let $L^{2}(\mathbb{G})$ be the GNS space for the (left) Haar weight of $\mathbb{G}$, so the reduced algebra $C_{0}(\mathbb{G})$ acts canonically on $L^{2}(\mathbb{G})$. Let $\pi: C_{0}^{u}(\mathbb{G}) \rightarrow C_{0}(\mathbb{G})$ be the "reducing morphism". There is a unitary corepresentation $\mathcal{V}$ of $C_{0}^{u}(\mathbb{G})$ on $L^{2}(\mathbb{G})$ such that $(\mathrm{id} \otimes \pi) \Delta_{u}(x)=\mathcal{V}^{*}(1 \otimes \pi(x)) \mathcal{V}$ for all $x \in C_{0}^{u}(\mathbb{G})$; see [23, Section 6].

With $\tilde{\Delta}_{u}: C_{0}^{u}(\mathbb{G})^{* *} \rightarrow C_{0}^{u}(\mathbb{G})^{* *} \bar{\otimes} C_{0}^{u}(\mathbb{G})^{* *}$ and $\tilde{\pi}: C_{0}^{u}(\mathbb{G})^{* *} \rightarrow C_{0}^{u}(\mathbb{G})$ being the normal extensions, arguing by weak*-continuity, we also have that

$$
(\mathrm{id} \otimes \tilde{\pi}) \tilde{\Delta}_{u}(x)=\mathcal{V}^{*}(1 \otimes \tilde{\pi}(x)) \mathcal{V} \quad\left(x \in C_{0}^{u}(\mathbb{G})^{* *}\right) .
$$

It then follows that

$$
\mathcal{V}_{12}^{*}(\tilde{\pi} \otimes \mathrm{id})(U)_{23} \mathcal{V}_{12}=(\mathrm{id} \otimes \tilde{\pi} \otimes \mathrm{id})\left(U_{13} U_{23}\right)=U_{13}(\tilde{\pi} \otimes \mathrm{id})(U)_{23}
$$

As $\mathcal{V} \in M\left(C_{0}^{u}(\mathbb{G}) \otimes \mathcal{B}_{0}\left(L^{2}(\mathbb{G})\right)\right)$, it follows that

$$
\begin{aligned}
(U(1 \otimes p))_{13} & =U_{13}(\tilde{\pi} \otimes \mathrm{id})\left(U U^{*}\right)_{23}=\mathcal{V}_{12}^{*}(\tilde{\pi} \otimes \mathrm{id})(U)_{23} \mathcal{V}_{12}(\tilde{\pi} \otimes \mathrm{id})(U)_{23}^{*} \\
& \in M\left(C_{0}^{u}(\mathbb{G}) \otimes \mathcal{B}_{0}\left(L^{2}(\mathbb{G})\right) \otimes \mathcal{B}_{0}\left(L^{2}(\mathbb{G})\right)\right) .
\end{aligned}
$$

Thus $U(1 \otimes p) \in M\left(C_{0}^{u}(\mathbb{G}) \otimes \mathcal{B}_{0}\left(L^{2}(G)\right)\right)$. If we represent $C_{0}^{u}(\mathbb{G})$ faithfully and non-degenerately on an auxiliary Hilbert space $K$, then we can regard $U$ as a partial isometry with initial and final spaces $K \otimes P(H)$, this is enough to show that also $U \in M\left(C_{0}^{u}(\mathbb{G}) \otimes \mathcal{B}_{0}\left(L^{2}(\mathbb{G})\right)\right)$.

Remark 3.20. In the proof of Proposition 3.17 we showed that in the commutative situation, a contractive corepresentation is necessarily a (possibly degenerate) unitary corepresentation. Unfortunately, we don't know this to be true for locally compact quantum groups, even in the cocommutative case, see [7] and [8]. Remark 3.21. Let $\mathcal{V} \in M\left(C_{0}^{u}(\mathbb{G}) \otimes \mathcal{B}_{0}\left(L^{2}(\mathbb{G})\right)\right.$ ) again denote the universal left regular representation, see 23, Section 6]. Then $\left(C_{0}^{u}(\mathbb{G}), \Delta_{u}, \mathcal{V}, L^{2}(\mathbb{G})\right)$ satisfies all the axioms for a $C^{*}$-Eberlein algebra; for axiom (2) see the discussion after [23, Proposition 5.1]. Hence the category of locally compact quantum groups forms a full sub-category of the $C^{*}$-Eberlein algebras. Unfortunately, due to the above comment, we have to restrict the natural morphisms between locally compact quantum groups and $C^{*}$-Eberlein algebras. 


\subsection{Banach algebra issues}

Let $(A, \Phi, V, H)$ be a $C^{*}$-Eberlein algebra, so that $A^{*}$ is a Banach algebra. We claim that it is a dual Banach algebra, meaning that the multiplication is separately weak ${ }^{*}$-continuous. Alternatively, we can always turn $A^{* *}$ into an $A^{*}$ bimodule via

$$
\langle x \cdot \mu, \lambda\rangle=\langle x, \mu \star \lambda\rangle, \quad\langle\mu \cdot x, \lambda\rangle=\langle x, \lambda \star \mu\rangle \quad\left(x \in A^{* *}, \mu, \lambda \in A^{*}\right) .
$$

Then $A^{*}$ is a dual Banach algebra if and only if $A$ is a submodule of $A^{* *}$.

Let us introduce some notation: for $\mu \in A^{*}$ let $V_{\mu}=(\mu \otimes \mathrm{id})(V) \in \mathcal{B}(H)$. By axiom (4), we know that $\mu \mapsto V_{\mu}$ is a representation of $A^{*}$. By axiom (2), this map $\mu \mapsto V_{\mu}$ is both injective, and weak ${ }^{*}$-weak*continuous.

Proposition 3.22. $A^{*}$ is a dual Banach algebra.

Proof. We verify that $A$ is a submodule of $A^{* *}$. By axiom (2), and continuity, it's enough to check that $A_{V}$ is a submodule. However, for $a=(\operatorname{id} \otimes \omega)(V) \in A$ and $\mu, \lambda \in A^{*}$,

$$
\langle a \cdot \mu, \lambda\rangle=\langle V, \mu \star \lambda \otimes \omega\rangle=\left\langle V_{\mu} V_{\lambda}, \omega\right\rangle=\left\langle V, \lambda \otimes \omega V_{\mu}\right\rangle
$$

so $a \cdot \mu=\left(\operatorname{id} \otimes \omega V_{\mu}\right)(V) \in A_{V}$. Similarly, one can check that $\mu \cdot a=\left(\operatorname{id} \otimes V_{\mu} \omega\right)(V) \in A_{V}$.

Alternatively, we note that $\mu \mapsto V_{\mu}$, when restricted to the closed unit ball of $A^{*}$, must be a homeomorphism onto its range (as the ball is weak ${ }^{*}$-compact). Then $\left\{V_{\mu}:\|\mu\| \leq 1\right\}$ is a weak*-compact sub-semigroup of $\mathcal{B}(H)_{\|\cdot\| \leq 1}$ and is hence semitopological. Thus $\left\{\mu \in A^{*}:\|\mu\| \leq 1\right\}$ with the product $\star$ is a semitopological semigroup, and the result follows.

Recall that if $A$ is a $\mathrm{C}^{*}$-algebra and $M(A)$ its multiplier algebra, then any $\mu \in A^{*}$ is strictly continuous, and so has an extension to $M(A)$. Alternatively, we can regard $M(A)$ as a (norm) closed subalgebra of $A^{* *}$, and then $A^{*}$ acts by restriction on $M(A)$. These two maps $A^{*} \rightarrow M(A)^{*}$ are the same. If $\theta: A_{2} \rightarrow M\left(A_{1}\right)$ is a morphism $\mathbb{S}_{1} \rightarrow \mathbb{S}_{2}$, we get an induced homomorphism $\theta^{*}: A_{1}^{*} \rightarrow A_{2}^{*}$; but this is a slight abuse of notation, as $\theta$ might not map into $A_{1}$, and so $\theta^{*}$ might not be weak ${ }^{*}$-continuous.

We now study some permanence properties of $C^{*}$-Eberlein algebras, making use of the above observations.

Proposition 3.23. Let $(A, \Phi, V, H)$ be a $C^{*}$-Eberlein algebra, let $B$ be a $C^{*}$-algebra, and let $\theta: A \rightarrow B$ be a surjection. Set $I$ to be the kernel of $\theta$, so $B \cong A / I$. Then there exists $\Phi^{\prime}: B \rightarrow B^{* *} \bar{\otimes} B^{* *}$ and $V^{\prime} \in B^{* *} \bar{\otimes} \mathcal{B}\left(H^{\prime}\right)$ making $\left(B, \Phi^{\prime}, V^{\prime}, H^{\prime}\right)$ into a $C^{*}$-Eberlein algebra with $\theta$ a morphism, if, and only if, $I^{\perp}$ is a subalgebra of $A^{*}$.

Proof. Note that $\theta^{*}: B^{*} \rightarrow A^{*}$ is an isometry onto its range, which is $I^{\perp}=\left\{\mu \in A^{*}:\langle\mu, a\rangle=0(a \in I)\right\}$. Then $\theta: A^{* *} \rightarrow B^{* *}$ is a surjection with kernel $I^{\perp \perp} \cong I^{* *}$. As $I^{\perp \perp}$ is an ideal in $A^{* *}$, there is a central projection $p \in A^{* *}$ with $(1-p) A^{* *}=I^{\perp \perp}$ and so $B^{* *} \cong A^{* *} / I^{\perp \perp}$ is isomorphic to $p A^{* *}$. Notice also that the map $\mu \mapsto p \mu$ defines a surjection $A^{*} \rightarrow I^{\perp}$.

If $\theta$ is a morphism, then $I^{\perp} \cong B^{*}$ is a subalgebra of $A^{*}$. Conversely, if $I^{\perp}$ is a subalgebra of $A^{*}$, then consider $\Phi^{\prime \prime}: A \rightarrow B^{* *} \bar{\otimes} B^{* *}, a \mapsto(p \otimes p) \Phi(a)$ which is a $*$-homomorphism as $p$ is central. Now let $\mu, \lambda \in A^{*}$ so that $p \mu, p \lambda \in I^{\perp}$ and so by assumption $p \mu \star p \lambda \in I^{\perp}$. Thus, for $a \in I$ we have that $0=\langle p \mu \star p \lambda, a\rangle=\langle(p \otimes p) \Phi(a), \mu \otimes \lambda\rangle$. This shows that $\Phi^{\prime \prime}(I)=\{0\}$ and so there is a well-defined *-homomorphism $\Phi^{\prime}: B=A / I \rightarrow B^{* *} \bar{\otimes} B^{* *}$ given by $\Phi^{\prime}(a+I)=\Phi^{\prime \prime}(a)=(p \otimes p) \Phi(a)$.

So $\Phi^{\prime}$ induces a product on $B^{*}$. Then, for $a \in A$ and $\mu, \lambda \in B^{*} \cong I^{\perp}$, we see that

$$
\begin{aligned}
\left\langle\theta^{*}(\mu \star \lambda), a\right\rangle & =\left\langle\Phi^{\prime}(\theta(a)), \mu \otimes \lambda\right\rangle=\langle(p \otimes p) \Phi(a), \mu \otimes \lambda\rangle=\langle\Phi(a), \mu \otimes \lambda\rangle=\langle\mu \star \lambda, a\rangle \\
& =\left\langle\theta^{*}(\mu) \star \theta^{*}(\lambda), a\right\rangle,
\end{aligned}
$$

as $\theta^{*}: B^{*} \cong I^{\perp} \rightarrow A^{*}$ is just the inclusion. 
Finally, we set $H^{\prime}=H$ and $V^{\prime}=(p \otimes 1) V \in B^{* *} \bar{\otimes} \mathcal{B}\left(H^{\prime}\right)$, so that for $\omega \in \mathcal{B}\left(H^{\prime}\right)_{*}$,

$$
(\mathrm{id} \otimes \omega)\left(V^{\prime}\right)=p(\mathrm{id} \otimes \omega)(V)=\theta^{* *}((\mathrm{id} \otimes \omega)(V))=\theta((\mathrm{id} \otimes \omega)(V)) \in B,
$$

as (id $\otimes \omega)(V) \in A$. As $\theta$ is onto, it follows that $\left\{(\mathrm{id} \otimes \omega)\left(V^{\prime}\right): \omega \in \mathcal{B}\left(H^{\prime}\right)_{*}\right\}$ is dense in $B$. Also $\Phi^{\prime}\left((\mathrm{id} \otimes \omega)\left(V^{\prime}\right)\right)=(p \otimes p) \Phi((\mathrm{id} \otimes \omega)(V))=(\mathrm{id} \otimes \mathrm{id} \otimes \omega)\left(V_{13}^{\prime} V_{23}^{\prime}\right)$ and so $V^{\prime}$ is a corepresentation, as required.

We remark that the "coproduct" $\Phi^{\prime}$ on $B$ is uniquely determined by the product on $B^{*}$. As $\theta: A \rightarrow B$ is onto, the coproduct $\Phi^{\prime}$ is hence unique.

Proposition 3.24. Let $\mathbb{S}_{1} \rightarrow \mathbb{S}_{2}$ be a morphism induced by $\theta: A_{2} \rightarrow M\left(A_{1}\right)$. With $I=\operatorname{ker} \theta$ we have that $I^{\perp}$ is a subalgebra of $A_{2}^{*}$ and so $B=\theta\left(A_{2}\right) \subseteq M\left(A_{1}\right)$ has the structure of a $C^{*}$-Eberlein algebra. The inclusion $B \rightarrow M\left(A_{1}\right)$ is a morphism.

Proof. That we have to work in $M\left(A_{1}\right)$ complicates matters. Let $\mu \in I^{\perp} \cong B^{*}$ so by Hahn-Banach there is $\mu_{0} \in M\left(A_{1}\right)^{*}$ extending $\mu$. Viewing $M\left(A_{1}\right)$ as a subalgebra of $A_{1}^{* *}$ there is $\mu_{1} \in A_{1}^{* * *}$ extending $\mu_{0}$ and so there is a bounded net $\left(\mu_{i}\right)$ in $A_{1}^{*}$ converging weak* to $\mu_{2}$. That is,

$$
\lim _{i}\left\langle\mu_{i}, \theta(a)\right\rangle=\langle\mu, a\rangle \quad\left(a \in A_{2}\right) .
$$

Similarly choose $\left(\lambda_{i}\right)$ for $\lambda \in I^{\perp}$. Then, for $a \in I$,

$$
\begin{aligned}
\langle\mu \star \lambda, a\rangle & =\langle\mu, \lambda \cdot a\rangle=\lim _{i}\left\langle\mu_{i}, \theta(\lambda \cdot a)\right\rangle=\lim _{i}\left\langle\theta^{*}\left(\mu_{i}\right) \star \lambda, a\right\rangle \\
& =\lim _{i}\left\langle\lambda, a \cdot \theta^{*}\left(\mu_{i}\right)\right\rangle=\lim _{i} \lim _{j}\left\langle\lambda_{j}, \theta\left(a \cdot \theta^{*}\left(\mu_{i}\right)\right)\right\rangle=\lim _{i, j}\left\langle\theta^{*}\left(\mu_{i}\right) \star \theta^{*}\left(\lambda_{j}\right), a\right\rangle \\
& =\lim _{i, j}\left\langle\theta^{*}\left(\mu_{i} \star \lambda_{j}\right), a\right\rangle=0,
\end{aligned}
$$

as $\theta(a)=0$. Thus $\mu \star \lambda \in I^{\perp}$ as claimed.

The inclusion $i: B \rightarrow M\left(A_{1}\right)$ is a $*$-homomorphism, and it is non-degenerate as $\operatorname{lin}\{b a: b \in A, a \in$ $\left.A_{1}\right\}=\operatorname{lin}\left\{\theta\left(a^{\prime}\right) a: a^{\prime} \in A_{2}, a \in A_{1}\right\}$ is dense in $A_{1}$ as $\theta$ is non-degenerate. Then $i$ is a morphism if and only if $i^{*}: A_{1}^{*} \rightarrow B^{*}$ is a homomorphism. However, $B^{*}$ is isomorphic (by construction, as a Banach algebra) to $I^{\perp} \subseteq A_{2}^{*}$ and $i^{*}$, when composed with this inclusion $B^{*} \rightarrow A_{2}^{*}$, is the map $\theta^{*}$ which is a homomorphism. So $i^{*}$ is a homomorphism as well, and $i$ is a morphism.

\subsection{Embedded $C^{*}$-Eberlein algebras}

We now start a process of thinking about compactifcations, which as we will see in Section 5 leads naturally to considering a locally compact quantum group $\mathbb{G}$ and $\mathrm{C}^{*}$-Eberlein algebras $(A, \Phi, V, H)$ with $A$ a subalgebra of $M\left(C_{0}^{u}(\mathbb{G})\right)$ and $\Phi$ being "induced" by the coproduct of $C_{0}^{u}(\mathbb{G})$. The purpose of this section is to make this concept precise, and to characterise when it can occur.

We start with a useful technical proposition.

Proposition 3.25. Let $A$ be a $C^{*}$-algebra, $H$ a Hilbert space, and $B \subseteq M(A)$ a sub-C ${ }^{*}$-algebra. Let

$$
X_{0}=\left\{T \in M\left(A \otimes \mathcal{B}_{0}(H)\right):(\mathrm{id} \otimes \omega)(T) \in B\left(\omega \in \mathcal{B}(H)_{*}\right)\right\} .
$$

Then $X_{0}$ is a closed, *-closed subspace of $M\left(A \otimes \mathcal{B}_{0}(H)\right)$. For each $T \in X_{0}$ there is $S \in B^{* *} \bar{\otimes} \mathcal{B}(H)$ with $(\mathrm{id} \otimes \omega)(T)=(\mathrm{id} \otimes \omega)(S) \in B \subseteq B^{* *}$ for all $\omega \in \mathcal{B}(H)_{*}$. If $T_{1}, T_{2} \in X_{0}$ are such that $T_{1} T_{2} \in X_{0}$, and $T_{i} \mapsto S_{i}$, then $T_{1} T_{2} \mapsto S_{1} S_{2}$. 
Proof. For $T \in X_{0}$, the map $\mathcal{B}(H)_{*} \rightarrow M(A) ; \omega \mapsto(\mathrm{id} \otimes \omega)(T)$ is completely bounded, and hence so is the corestriction $\mathcal{B}(H)_{*} \rightarrow B$, and hence so is the inclusion $B \subseteq B^{* *}$, which finally induces $S \in B^{* *} \bar{\otimes} \mathcal{B}(H)$. We have that $\|S\|=\|T\|$. We first claim that this map is a $*$-map. Notice that $($ id $\otimes \omega)\left(T^{*}\right)=\left(\operatorname{id} \otimes \omega^{*}\right)(T)^{*} \in$ $B$ so $T^{*} \in X_{0}$. Then, for $\mu \in B^{*}$,

$$
\left\langle\mu,(\operatorname{id} \otimes \omega)\left(S^{*}\right)\right\rangle=\overline{\left\langle S, \mu^{*} \otimes \omega^{*}\right\rangle}=\overline{\left\langle\mu^{*},\left(\operatorname{id} \otimes \omega^{*}\right)(T)\right\rangle}=\left\langle\mu,(\mathrm{id} \otimes \omega)\left(T^{*}\right)\right\rangle,
$$

so $T^{*} \mapsto S^{*}$ as claimed.

Now let $T_{1}, T_{2} \in X_{0}$ map to $S_{1}, S_{2}$. Let $\left(e_{i}\right)$ be an orthonormal basis of $H$. For $\xi, \eta \in H$,

$$
\left(\mathrm{id} \otimes \omega_{\xi, \eta}\right)\left(T_{1} T_{2}\right)=\sum_{i}\left(\mathrm{id} \otimes \omega_{\xi, \eta}\right)\left(T_{1}\left(1 \otimes \theta_{e_{i}, e_{i}}\right) T_{2}\right)=\sum_{i}\left(\mathrm{id} \otimes \omega_{e_{i}, \eta}\right)\left(T_{1}\right)\left(\mathrm{id} \otimes \omega_{\xi, e_{i}}\right)\left(T_{2}\right) .
$$

This sum converges in the strict topology on $M(A)$; compare with the discussion in [25, Section 5.5], or [25. Lemma A.5], for example. However, each summand is a member of $B \subseteq B^{* *}$ and one can similarly prove that the sum converges in the $\sigma$-weak topology on $B^{* *}$, compare with Section 3.3 above. Note in passing that we don't see why the sum need converge in norm in $B$, and so there is no reason why $X=X_{0}$. Continuing, we see that in $B^{* *}$, the sum is also equal to

$$
\sum_{i}\left(\mathrm{id} \otimes \omega_{e_{i}, \eta}\right)\left(S_{1}\right)\left(\mathrm{id} \otimes \omega_{\xi, e_{i}}\right)\left(S_{2}\right)=\left(\mathrm{id} \otimes \omega_{\xi, \eta}\right)\left(S_{1} S_{2}\right) .
$$

By assumption, $T_{1} T_{2} \in X_{0}$ and so $(\mathrm{id} \otimes \omega)\left(T_{1} T_{2}\right) \in B$. The argument now shows this to be equal to $($ id $\otimes \omega)\left(S_{1} S_{2}\right)$ as required.

Remark 3.26. We shall apply this proposition in the case when $B$ is unital. Then, for example, if $U \in X_{0}$ is unitary, then $U^{*} U=U U^{*}=1_{A} \otimes I_{H}=1_{B} \otimes I_{H}$ and so the 2nd part of the proposition allows us to conclude that the image of $U$ in $B^{* *} \bar{\otimes} \mathcal{B}(H)$ is also unitary.

In general, the sum may indeed fail to converge in norm. For example, if $A=B=\mathcal{B}_{0}(H)$ then $M\left(A \otimes \mathcal{B}_{0}(H)\right)=M\left(B_{0}(H) \otimes \mathcal{B}_{0}(H)\right) \cong \mathcal{B}(H \otimes H)$. Let $\Sigma \in \mathcal{B}(H \otimes H)$ be the tensor swap map, so for $\xi, \eta, \alpha, \beta \in H$,

$$
\left(\left(\mathrm{id} \otimes \omega_{\xi, \eta}\right)(\Sigma) \alpha \mid \beta\right)=(\Sigma(\alpha \otimes \xi) \mid \beta \otimes \eta)=(\xi \otimes \alpha \mid \beta \otimes \eta)=(\xi \mid \beta)(\alpha \mid \eta)=\left(\theta_{\xi, \eta} \alpha \mid \beta\right),
$$

and so $\left(\right.$ id $\left.\otimes \omega_{\xi, \eta}\right)(\Sigma)=\theta_{\xi, \eta} \in B$. So $\Sigma \in X_{0}$. However, $\Sigma^{2}=I_{H} \otimes I_{H}$ and so $\Sigma^{2} \notin X_{0}$.

Suppose we start with a locally compact quantum group $\mathbb{G}$ and a $C^{*}$-Eberlein algebra $\mathbb{S}=(A, \Phi, V, H)$ together with a morphism $\mathbb{G} \rightarrow \mathbb{S}$ given by $\theta: A \rightarrow M\left(C_{0}^{u}(\mathbb{G})\right)$. If $B=\theta(A) \subseteq M\left(C_{0}^{u}(\mathbb{G})\right)$ then by Proposition 3.24 we can give $B$ a unique structure as a $C^{*}$-Eberlein algebra, in such a way that the inclusion $\phi: B \rightarrow M\left(C_{0}^{u}(\mathbb{G})\right)$ is a morphism. Indeed, this uses Proposition 3.23 to form $\left(B, \Phi_{B}, V_{B}, H\right)$ with $V_{B}=\left(\theta_{0}^{* *} \otimes \mathrm{id}\right)(V) \in B^{* *} \bar{\otimes} \mathcal{B}(H)$, where $\theta_{0}: A \rightarrow B$ is the corestriction of $\theta$. Let us pin down the "corepresentation" $V_{B}$ a little further.

Proposition 3.27. Continuing with the notation of the previous paragraph, there is a subspace $K$ of $H$ which is invariant for $V_{B}$ and such that if $U^{\prime}$ is the restriction of $V_{B}$ to $K$ then $U^{\prime}$ is unitary and $\left(B, \Phi_{B}, U^{\prime}, K\right)$ is a $C^{*}$-Eberlein algebra. Furthermore, there is a unitary corepresentation $U$ of $C_{0}^{u}(\mathbb{G})$ on $K$ such that

$$
\left\{(\operatorname{id} \otimes \omega)(U): \omega \in \mathcal{B}(K)_{*}\right\}
$$

is dense in $B$, and $U^{\prime}$ is generated from $U$ by the procedure of Proposition 3.25.

Proof. By the definition of $\theta$ being a morphism, $U_{0}=(\tilde{\theta} \otimes \mathrm{id})(V) \in C_{0}^{u}(\mathbb{G})^{* *} \bar{\otimes} \mathcal{B}(H)$ is such that $U_{0}^{*} U_{0}=$ $U_{0} U_{0}^{*}=1 \otimes p$ for some projection $p$ on $H$. By Lemma 3.19 we know that actually $U_{0} \in M\left(C_{0}^{u}(\mathbb{G}) \otimes \mathcal{B}(H)\right)$, so we may let $U$ be the restriction of $U_{0}$ to a unitary corepresentation of $C_{0}^{u}(\mathbb{G})$ in $M\left(C_{0}^{u}(\mathbb{G}) \otimes \mathcal{B}_{0}(K)\right)$, where $K=p(H)$. 
Now let $\tilde{\phi}: B^{* *} \rightarrow C_{0}^{u}(\mathbb{G})^{* *}$ be the normal extension of $\phi$ and notice that $\tilde{\phi} \circ \theta_{0}^{* *}=\tilde{\theta}$. Hence $(\tilde{\phi} \otimes \mathrm{id})\left(V_{B}\right)=U_{0}$. If $i: K \rightarrow H$ is the inclusion, then

$$
(\mathrm{id} \otimes \omega)(U)=(\operatorname{id} \otimes i \omega p)\left(U_{0}\right)=\tilde{\theta}((\mathrm{id} \otimes i \omega p)(V))=\theta((\operatorname{id} \otimes i \omega p)(V)) \quad\left(\omega \in \mathcal{B}(K)_{*}\right)
$$

and this is a member of $B$ by axiom (2). Furthermore, $B$ is in fact the closure of the set $\{($ id $\otimes \omega)(U)$ : $\left.\omega \in \mathcal{B}(K)_{*}\right\}$. So can apply the procedure of Proposition 3.25 to $U$ to yield $U^{\prime} \in B^{* *} \bar{\otimes} \mathcal{B}(K)$. By construction, $(\mathrm{id} \otimes \omega)\left(U^{\prime}\right)=(\mathrm{id} \otimes \omega)(U)=\tilde{\phi}\left((\mathrm{id} \otimes i \omega p)\left(V_{B}\right)\right)=\phi\left((\mathrm{id} \otimes i \omega p)\left(V_{B}\right)\right)$ as $(\mathrm{id} \otimes i \omega p)\left(V_{B}\right) \in B$, for any $\omega \in B(K)_{*}$. It follows that $U^{\prime}$ is simply the compression of $V_{B}$ to $K$, that is, $U^{\prime}=(1 \otimes p) V_{B}(1 \otimes i)$.

However, by Proposition 3.25, as $U^{*} U=U U^{*}=I$, also $U^{\prime}$ is unitary. So $K$ is an invariant subspace of $V_{B}$, and hence $\left(B, \Phi_{B}, U^{\prime}, K\right)$ is a $C^{*}$-Eberlein algebra.

Let us think when we can reverse this process: that is, if $U \in M\left(C_{0}^{u}(\mathbb{G}) \otimes \mathcal{B}(K)\right)$ is a unitary corepresentation of $C_{0}^{u}(\mathbb{G})$, when is there a $C^{*}$-Eberlein algebra $(A, \Phi, V, H)$ and a morphism $\theta: A \rightarrow$ $M\left(C_{0}^{u}(\mathbb{G})\right)$ such that $U$ arises from the construction above? It is clearly necessary that $\{($ id $\otimes \omega)(U): \omega \in$ $\left.\mathcal{B}(K)_{*}\right\}$ is dense in a $C^{*}$-algebra $B \subseteq M\left(C_{0}^{u}(\mathbb{G})\right.$ ) (which will be the image of $\theta$ ). This turns out to also be sufficient.

Theorem 3.28. Let $U \in M\left(C_{0}^{u}(\mathbb{G}) \otimes \mathcal{B}(K)\right)$ be a unitary corepresentation of $C_{0}^{u}(\mathbb{G})$ such that the closure of $\left\{(\operatorname{id} \otimes \omega)(U): \omega \in \mathcal{B}(K)_{*}\right\}$ is a $C^{*}$-algebra $B \subseteq M\left(C_{0}^{u}(\mathbb{G})\right)$. Let $U^{\prime} \in B^{* *} \bar{\otimes} \mathcal{B}(K)$ be given by Proposition 3.25 applied to $U$. Then there is a unique $\Phi_{B}$ such that $\mathbb{S}=\left(B, \Phi_{B}, U^{\prime}, K\right)$ is a $C^{*}$-Eberlein algebra with the inclusion $B \rightarrow M\left(C_{0}^{u}(\mathbb{G})\right)$ inducing a morphism $\mathbb{G} \rightarrow \mathbb{S}$.

Before giving the proof, we need to introduce a little Banach algebraic background. For a Banach algebra $\mathfrak{A}$ turn $\mathfrak{A}^{*}$ into an $\mathfrak{A}$-bimodule in the usual way. For a functional $\mu \in \mathfrak{A}^{*}$, define the orbit maps

$$
L_{\mu}: \mathfrak{A} \rightarrow \mathfrak{A}^{*}, a \mapsto \mu \cdot a, \quad R_{\mu}: \mathfrak{A} \rightarrow \mathfrak{A}^{*}, a \mapsto a \cdot \mu .
$$

Let $\kappa: \mathfrak{A} \rightarrow \mathfrak{A}^{* *}$ be the canonical map from a Banach space to its bidual. Then it's easy to see that $L_{\mu}^{*} \circ \kappa=R_{\mu}$ and $R_{\mu}^{*} \circ \kappa=L_{\mu}$. From Gantmacher's theorem, it follows that $R_{\mu}$ is a weakly-compact map if and only if $L_{\mu}$ is, and in this case we say that $\mu$ is weakly almost periodic, written $\mu \in \operatorname{wap}(\mathfrak{A})$. Then wap $(\mathfrak{A})$ is a closed (two-sided) submodule of $\mathfrak{A}^{*}$.

Now recall the Arens products on $\mathfrak{A}^{* *}$ : these are the two natural algebra products on $\mathfrak{A}^{* *}$ obtained by extending the product from $\mathfrak{A}$ by weak*-continuity, either on the left or the right. More algebraically, for $\Phi, \Psi \in \mathfrak{A}^{* *}$ and $\mu \in \mathfrak{A}, a \in \mathfrak{A}$ we successively define

$$
\begin{aligned}
\langle\Phi \square \Psi, \mu\rangle & =\langle\Phi, \Psi \cdot \mu\rangle, & & \langle\Phi \diamond \Psi, \mu\rangle=\langle\Psi, \mu \cdot \Phi\rangle \\
\langle\Psi \cdot \mu, a\rangle & =\langle\Psi, \mu \cdot a\rangle, & & \langle\mu \cdot \Phi, a\rangle=\langle\Phi, a \cdot \mu\rangle .
\end{aligned}
$$

Then $\square, \diamond$ are Banach algebra products on $\mathfrak{A}^{* *}$ making $\kappa$ a homomorphism. We say that a closed submodule $X$ of $\mathfrak{A}^{*}$ is introverted if $\Phi \cdot \mu, \mu \cdot \Phi \in X$ for all $\mu \in X, \Phi \in \mathfrak{A}^{* *}$. In this case, it is easy to see that $\square$ and $\diamond$ drop to well-defined products on $X^{*}=\mathfrak{A}^{* *} / X^{\perp}$. One can then show that the resulting products on $X^{*}$ are the same if and only if $X \subseteq \operatorname{wap}(\mathfrak{A})$ (and we have that wap( $\left.\mathfrak{A}\right)$ is always introverted). Furthermore, in this case, the product in $X^{*}$ is separately weak ${ }^{*}$-continuous, and so $X^{*}$ becomes a dual Banach algebra. For a self-contained proof of this, see [11, Proposition 2.4]; compare [27, Lemma 1.4].

Proof of Theorem 3.28. Firstly, $\theta: B \rightarrow M\left(C_{0}^{u}(\mathbb{G})\right)$ will be non-degenerate. Indeed, as $U$ is a unitary multiplier, $\left\{U(a \otimes \theta): a \in C_{0}^{u}(\mathbb{G}), \theta \in \mathcal{B}_{0}(K)\right\}$ will have dense linear span in $C_{0}^{u}(\mathbb{G}) \otimes \mathcal{B}_{0}(K)$ and hence

$$
\begin{aligned}
& \varlimsup\left\{(\operatorname{id} \otimes \omega)(U) a: a \in C_{0}^{u}(\mathbb{G}), \omega \in \mathcal{B}(K)_{*}\right\} \\
& =\varlimsup\left\{(\operatorname{id} \otimes \theta \omega)(U(a \otimes 1)): a \in C_{0}^{u}(\mathbb{G}), \theta \in \mathcal{B}_{0}(K), \omega \in \mathcal{B}(K)_{*}\right\} \\
& =\varlimsup\left\{(\mathrm{id} \otimes \omega)(U(a \otimes \theta)): a \in C_{0}^{u}(\mathbb{G}), \theta \in \mathcal{B}_{0}(K), \omega \in \mathcal{B}(K)_{*}\right\} \\
& =\varlimsup\left\{(\mathrm{id} \otimes \omega)(a \otimes \theta): a \in C_{0}^{u}(\mathbb{G}), \theta \in \mathcal{B}_{0}(K), \omega \in \mathcal{B}(K)_{*}\right\}=C_{0}^{u}(\mathbb{G}) \text {, }
\end{aligned}
$$


as required to show that $\theta(B) C_{0}^{u}(\mathbb{G})$ has dense span in $C_{0}^{u}(\mathbb{G})$.

We have that $C_{0}^{u}(\mathbb{G})^{*}$ is a Banach algebra, and so $C_{0}^{u}(\mathbb{G})^{* *}$ is a $C_{0}^{u}(\mathbb{G})^{*}$-bimodule. If we regard $B$ as a subalgebra of $M\left(C_{0}^{u}(\mathbb{G})\right)$, which in turn we regard as a subalgebra of $C_{0}^{u}(\mathbb{G})^{* *}$, then we claim that $B$ is a $C_{0}^{u}(\mathbb{G})^{*}$-submodule. Indeed, for $b=(\mathrm{id} \otimes \omega)(U) \in B$ and $\mu \in C_{0}^{u}(\mathbb{G})^{*}$, we find that for all $\lambda \in C_{0}^{u}(\mathbb{G})^{*}$,

$$
\langle\mu \cdot b, \lambda\rangle=\langle\lambda \star \mu \otimes \omega, U\rangle=\left\langle\lambda \otimes \mu \otimes \omega, U_{13} U_{23}\right\rangle=\left\langle\lambda \otimes \omega, U\left(1 \otimes\left(U_{\mu}\right)\right)\right\rangle=\left\langle\left(\operatorname{id} \otimes U_{\mu} \omega\right)(U), \lambda\right\rangle,
$$

so $\mu \cdot b=\left(\operatorname{id} \otimes U_{\mu} \omega\right)(U) \in B$. Similarly $b \cdot \mu=\left(\right.$ id $\left.\otimes \omega U_{\mu}\right)(U) \in B$. We next claim that $B \subseteq \operatorname{wap}\left(C_{0}^{u}(\mathbb{G})^{*}\right)$. Indeed, for $b$ of the form above, the map $C_{0}^{u}(\mathbb{G})^{*} \rightarrow B ; \mu \mapsto \mu \cdot b=\left(\operatorname{id} \otimes \omega U_{\mu}\right)(U)$ factors through the map $\mathcal{B}(H) \rightarrow \mathcal{B}(H)_{*} ; T \mapsto \omega T$ which is weakly-compact. As wap $\left(C_{0}^{u}(\mathbb{G})^{*}\right)$ is closed, the claim follows.

From the discussion above, the Arens products on $C_{0}^{u}(\mathbb{G})^{* * *}$ drop to a well-defined product on $B^{*}=$ $C_{0}^{u}(\mathbb{G})^{* * *} / B^{\perp}$ turning $B^{*}$ into a dual Banach algebra. As $C_{0}^{u}(\mathbb{G})^{*}$ is a completely contractive Banach algebra (it is the predual of the Hopf-von Neumann algebra $\left.\left(C_{0}^{u}(\mathbb{G})^{* *}, \tilde{\Delta}_{u}\right)\right)$ we may work in the category of Operator Spaces to conclude that $B^{*}$ is also a completely contractive dual Banach algebra. That is, the product on $B^{*}$ induces a complete contraction $\Phi_{*}: B^{*} \widehat{\otimes} B^{*} \rightarrow B^{*}$ and the adjoint gives a complete contraction $B^{* *} \rightarrow B^{* *} \bar{\otimes} B^{* *}$; let $\Phi_{B}$ be this map restricted to $B$.

We can describe $\Phi_{B}$ more concretely as follows. Let $\mu \in B^{*}$ and let $\mu_{0} \in C_{0}^{u}(\mathbb{G})^{* * *}$ be a Hahn-Banach extension. There is hence a bounded net $\left(\mu_{i}\right)$ in $C_{0}^{u}(\mathbb{G})^{*}$ converging weak* to $\mu_{0}$. For $\lambda \in B^{*}$ similarly find $\left(\lambda_{j}\right)$. Then, for $b \in B$,

$$
\left\langle\Phi_{B}(b), \mu \otimes \lambda\right\rangle=\langle\mu \star \lambda, b\rangle=\left\langle\mu_{0}, \lambda_{0} \cdot b\right\rangle=\lim _{i}\left\langle\lambda_{0} \cdot b, \mu_{i}\right\rangle=\lim _{i}\left\langle\lambda_{0}, b \cdot \mu_{i}\right\rangle=\lim _{i} \lim _{j}\left\langle b, \mu_{i} \star \lambda_{j}\right\rangle .
$$

We now use this to show that $\Phi_{B}$ is a $*$-homomorphism. From the above,

$$
\left\langle\Phi_{B}\left(b^{*}\right), \mu \otimes \lambda\right\rangle=\lim _{i} \lim _{j}\left\langle b^{*}, \mu_{i} \star \lambda_{j}\right\rangle=\lim _{i} \lim _{j} \overline{\left\langle b, \mu_{i}^{*} \star \lambda_{j}^{*}\right\rangle}=\overline{\left\langle\Phi(b), \mu^{*} \otimes \lambda^{*}\right\rangle}=\left\langle\Phi_{B}(b)^{*}, \mu \otimes \lambda\right\rangle .
$$

That $\left(\mu_{i} \star \lambda_{j}\right)^{*}=\mu_{i}^{*} \star \lambda_{j}^{*}$ follows as $\Delta_{u}$ is a $*$-homomorphism. So $\Phi_{B}$ is a $*$-map.

We now proceed to check that $\Phi_{B}$ is multiplicative, for which we "play-off" $U$ and $U^{\prime}$. By construction, $(\mathrm{id} \otimes \omega)\left(U^{\prime}\right)=(\mathrm{id} \otimes \omega)(U) \in B$ for all $\omega \in \mathcal{B}(H)_{*}$. For $\mu, \lambda \in B^{*}$ choose $\left(\mu_{i}\right),\left(\lambda_{i}\right)$ as above, and let $\omega \in \mathcal{B}(H)_{*}$. Then

$$
\begin{aligned}
\left\langle U_{\mu}^{\prime} U_{\lambda}^{\prime}, \omega\right\rangle & =\left\langle\mu,\left(\operatorname{id} \otimes U_{\lambda}^{\prime} \omega\right)(U)\right\rangle=\lim _{i}\left\langle\left(\operatorname{id} \otimes U_{\lambda}^{\prime} \omega\right)(U), \mu_{i}\right\rangle=\lim _{i}\left\langle U_{\lambda}^{\prime}, \omega U_{\mu_{i}}\right\rangle \\
& =\lim _{i}\left\langle\lambda,\left(\operatorname{id} \otimes \omega U_{\mu_{i}}\right)(U)\right\rangle=\lim _{i} \lim _{j}\left\langle U_{\lambda_{j}}, \omega U_{\mu_{i}}\right\rangle=\lim _{i} \lim _{j}\left\langle U_{\mu_{i}} U_{\lambda_{j}}, \omega\right\rangle \\
& =\lim _{i} \lim _{j}\left\langle U_{\mu_{i} \star \lambda_{j}}, \omega\right\rangle=\lim _{i} \lim _{j}\left\langle(\operatorname{id} \otimes \omega)(U), \mu_{i} \star \lambda_{j}\right\rangle=\left\langle\Phi_{B}((\operatorname{id} \otimes \omega)(U)), \mu \otimes \lambda\right\rangle .
\end{aligned}
$$

We conclude $\Phi_{B}\left((\mathrm{id} \otimes \omega)\left(U^{\prime}\right)\right)=(\mathrm{id} \otimes \mathrm{id} \otimes \omega)\left(U_{13}^{\prime} U_{23}^{\prime}\right)$. Notice also that

$$
\left\langle U_{\mu}^{\prime}, \omega\right\rangle=\left\langle\mu,(\operatorname{id} \otimes \omega)\left(U^{\prime}\right)\right\rangle=\lim _{i}\left\langle(\operatorname{id} \otimes \omega)(U), \mu_{i}\right\rangle=\lim _{i}\left\langle U_{\mu_{i}}, \omega\right\rangle,
$$

so $U_{\mu_{i}} \rightarrow U_{\mu}^{\prime}$ weak ${ }^{*}$ in $\mathcal{B}(H)$.

We now use the extended Haagerup tensor product, compare Section [3.3. Let $b=\left(\right.$ id $\left.\otimes \omega_{\xi, \eta}\right)(U) \in B$, and let $\left(e_{i}\right)$ be an orthonormal basis of $H$, so that, as in the previous paragraph

$$
\begin{aligned}
\left\langle\Phi_{B}(b), \mu \otimes \lambda\right\rangle & =\lim _{i} \lim _{j}\left\langle U_{\mu_{i}} U_{\lambda_{j}}, \omega\right\rangle=\lim _{i}\left\langle U_{\mu_{i}} U_{\lambda}^{\prime}, \omega\right\rangle=\left\langle U_{\mu}^{\prime} U_{\lambda}^{\prime}, \omega\right\rangle \\
& =\sum_{k}\left(U_{\mu}^{\prime} e_{k} \mid \eta\right)\left(U_{\lambda}^{\prime} \xi \mid e_{k}\right)=\sum_{k}\left\langle\mu,\left(\operatorname{id} \otimes \omega_{e_{k}, \eta}\right)(U)\right\rangle\left\langle\lambda,\left(\operatorname{id} \otimes \omega_{\xi, e_{k}}\right)(U)\right\rangle .
\end{aligned}
$$

Thus $\Phi_{B}(b)=\sum_{i}\left(\mathrm{id} \otimes \omega_{e_{i}, \eta}\right)(U) \otimes\left(\mathrm{id} \otimes \omega_{\xi, e_{i}}\right)(U)$ a sum which converges in $B \otimes^{e h} B \subseteq B^{* *} \otimes^{e h} B^{* *}$ treated as a (not closed) subspace of $B^{* *} \bar{\otimes} B^{* *}$. However, we could alternatively treat $B$ as a subalgebra of $M\left(C_{0}^{u}(\mathbb{G})\right)$ and in turn treat this as a subalgebra of $C_{0}(\mathbb{G})^{* *}$. Exactly the same argument then shows 
that $\sum_{i}\left(\mathrm{id} \otimes \omega_{e_{i}, \eta}\right)(U) \otimes\left(\mathrm{id} \otimes \omega_{\xi, e_{i}}\right)(U)$ converges weak* in $C_{0}^{u}(\mathbb{G})^{* *} \otimes{ }^{e h} C_{0}^{u}(\mathbb{G})^{* *}$ and is equal to $\Delta_{u}(b)$, identifying $M\left(C_{0}^{u}(\mathbb{G}) \otimes C_{0}^{u}(\mathbb{G})\right)$ with a subalgebra of $C_{0}^{u}(\mathbb{G})^{* *} \bar{\otimes} C_{0}^{u}(\mathbb{G})^{* *}$.

By [16, Lemma 5.4] we know that the extended Haagerup tensor product is "injective". In particular, thinking of $B$ as a subalgebra of $C_{0}^{u}(\mathbb{G})^{* *}$, the map $B \otimes^{e h} B \rightarrow C_{0}^{u}(\mathbb{G})^{* *} \otimes^{e h} C_{0}^{u}(\mathbb{G})^{* *}$ is a complete isometry. Using the arguments of [16, Sections 6-7] (a result proved for von Neumann algebras in [6]) we know that $B \otimes^{e h} B$ is an algebra. Thus, if we set $X=\left\{(\operatorname{id} \otimes \omega)(U): \omega \in \mathcal{B}(H)_{*}\right\}$ then $X$ is a dense subalgebra of $B$, and $\Phi_{B}$ restricts to a map $X \rightarrow B \otimes^{e h} B$. Under the identifications derived above, $\Phi_{B}$ is equal to $\Delta_{u}$ regarded as a map $X \rightarrow B \otimes^{\text {eh }} B$. As $\Delta_{u}$ is homomorphism, so is $\Phi_{B}$, restricted to $X$. By continuity, $\Phi_{B}: B \rightarrow B^{* *} \bar{\otimes} B^{* *}$ is a homomorphism, as required.

In summary, we have that $\mathbb{S}=\left(B, \Phi_{B}, U^{\prime}, H\right)$ is a $C^{*}$-Eberlein algebra and the inclusion $\theta: B \rightarrow$ $M\left(C_{0}^{u}(\mathbb{G})\right)$ is a non-degenerate $*$-homomorphism. By construction, $\theta^{*}: C_{0}^{u}(\mathbb{G})^{*} \rightarrow B^{*}$ is a homomorphism, and so $\theta$ defines a morphism $\mathbb{S} \rightarrow \mathbb{G}$.

Definition 3.29. $C^{*}$-Eberlein algebras $\left(B, \Phi_{B}, U^{\prime}, H\right)$ which arise in this way are embedded $C^{*}$-Eberlein algebras of $\mathbb{G}$.

Remark 3.30. Let us just remark that we used very little of the structure of locally compact quantum groups here: the results would make sense of any $C^{*}$-bialgebra $(A, \Delta)$ replacing $C_{0}^{u}(\mathbb{G})$, with the appropriate notion of a "morphism".

In particular, the theory would work with $C_{0}^{u}(\mathbb{G})$ replaced by $C_{0}(\mathbb{G})$. However, there is a subtle point here. Any unitary corepresentation $U$ of $C_{0}(\mathbb{G})$ "lifts" uniquely to a corepresentation $V$ of $C_{0}^{u}(\mathbb{G})$, see [23, Proposition 6.6]. If $V$ satisfies the condition of Theorem 3.28 above, then so does $U$, as we can just apply the canonical surjection $C_{0}^{u}(\mathbb{G}) \rightarrow C_{0}(\mathbb{G})$. However, we have been unable to decide if the converse to this claim holds.

\section{Invariant means}

Classically, the existance of invariant means is proved by use of a fixed point theorem (for example, the use of the Ryll-Nardzewski Theorem in the proof of [4, Theorem 2.15, Chapter 4]). Instead, we shall use a Hilbert space technique.

We proceed with some generality. Let $A$ be a $C^{*}$-algebra with $\Phi: A \rightarrow A^{* *} \bar{\otimes} A^{* *}$ a unital *homomorphism inducing a Banach algebra product on $A^{*}$. Let $U \in A^{* *} \bar{\otimes} \mathcal{B}(H)$ be a corepresentation of $(A, \Phi)$, in the sense of Remark 3.7 .

Definition 4.1. A vector $\xi \in H$ is invariant for $U$ if $(\mu \otimes \iota)(U) \xi=\xi\langle\mu, 1\rangle$ for all $\mu \in A^{*}$. Denote by $\operatorname{Inv}(U)$ the collection of all invariant vectors of $U$.

For $\xi \in H$, the average of $\xi$ is the unique vector of minimal norm in the closure of the convex set $C_{\xi}:=\{(\mu \otimes \iota)(U) \xi: \mu$ a state on $A\}$, which exists by properties of Hilbert spaces.

Proposition 4.2. Let $U$ be a contractive corepresentation of $A$ on $H$, and let $\xi \in H$. The average of $\xi$ is invariant for $U$.

Proof. Let $\xi_{0}$ be the average of $\xi$. If $\mu, \lambda \in A^{*}$ are states, then so is $\mu \star \lambda$, as $\Phi$ is a unital $*$-homomorphism. Hence $(\mu \otimes \mathrm{id})(U)(\lambda \otimes \mathrm{id})(U) \xi=(\mu \star \lambda \otimes \mathrm{id})(U) \xi \in C_{\xi}$. It follows by continuity that $(\mu \otimes \mathrm{id})(U)$ leaves $C_{\xi}$ invariant. As $U$ is contractive, if $\mu \in A^{*}$ is a state, then $(\mu \otimes \mathrm{id})(U)$ is a contractive operator. Hence $(\mu \otimes \mathrm{id})(U) \xi_{0} \in C_{\xi}$ and $\left\|(\mu \otimes \mathrm{id})(U) \xi_{0}\right\| \leq\left\|\xi_{0}\right\|$. By uniqueness, $(\mu \otimes \mathrm{id})(U) \xi_{0}=\xi_{0}$. This holds for all states, so by polar decomposition, $(\mu \otimes \mathrm{id})(U) \xi_{0}=\langle\mu, 1\rangle \xi_{0}$ for all $\mu \in A^{*}$, as claimed.

We now proceed to construct means on $\mathrm{C}^{*}$-Eberlein algebras. We are inspired by [4, Chapter 2.3], but we shall work with the language of Banach algebras. Let $\mathbb{S}=(A, \Phi, V, H)$ be a $C^{*}$-Eberlein algebra, where in this section we shall suppose that $A$ is unital. Recall from Proposition 3.22 that $A^{*}$ becomes a dual Banach algebra, and so $A$ is an $A^{*}$-bimodule. 
Definition 4.3. A state $M \in A^{*}$ is a left invariant mean if $\langle M, a \cdot \mu\rangle=\langle M, a\rangle$ for all states $\mu \in A^{*}$ and $a \in A$. Similarly we defined a right invariant mean. A mean which is both left and right invariant is an invariant mean.

As $\Phi: A \rightarrow A^{* *} \bar{\otimes} A^{* *}$ we can make sense of the map (id $\left.\otimes M\right) \Phi: A \rightarrow A^{* *}$ (which will be completely positive). Then $M$ is left invariant if and only if (id $\otimes M) \Phi$ is the rank-one map $a \mapsto\langle M, a\rangle$; similarly $M$ is right invariant if $(M \otimes$ id $) \Phi$ is the rank-one map $a \mapsto\langle M, a\rangle 1$.

Theorem 4.4. For $a \in A$, let $K(a)=\{\mu \cdot a: \mu$ is a state on $A\}$. Then the following are equivalent:

1. A has a left invariant mean;

2. $K(a) \cap \mathbb{C} 1 \neq \emptyset$ for all $a \in A$;

3. $0 \in K(a-a \cdot \mu)$ for all $a \in A$ and all states $\mu \in A^{*}$.

Proof. This is an abstract version of [4, Theorem 2.3.11]. By definition, $M \in A^{*}$ is left invariant when $\mu \star M=\langle\mu, 1\rangle M$ for all $\mu \in A^{*}, a \in A$, or equivalently, if $M \cdot a=\langle M, a\rangle 1$ for all $a \in A$. So (11) $\Rightarrow(2)$. If (2) holds then for $a \in A$ there is a state $\mu \in A^{*}$ and $t \in \mathbb{C}$ with $\mu \cdot a=t 1$. Then for a state $\lambda \in A^{*}$ we have that $\mu \cdot(a-a \cdot \lambda)=t 1-(\mu \cdot a) \cdot \lambda=t 1-t 1 \cdot \lambda=0$ as $1 \cdot \lambda=(\lambda \otimes$ id $) \Phi(1)=1$. So (2) $\Rightarrow(3)$.

Now suppose that (3) holds. For each $a \in A$ and state $\mu$ let

$$
M(a, \mu)=\left\{\lambda \in A^{*} \text { a state }: \lambda \cdot(a-a \cdot \mu)=0\right\},
$$

which is non-empty by assumption. If $\left(\lambda_{\alpha}\right)$ is a net in $M(a, \mu)$ converging weak* to $\lambda$, then $\lambda$ is a state, and for any $\phi \in A^{*}$,

$$
\langle\phi, \lambda \cdot(a-a \cdot \mu)\rangle=\langle\lambda,(a-a \cdot \mu) \cdot \phi\rangle=\lim _{\alpha}\left\langle\lambda_{\alpha},(a-a \cdot \mu) \cdot \phi\right\rangle=\lim _{\alpha}\left\langle\phi, \lambda_{\alpha} \cdot(a-a \cdot \mu)\right\rangle=0 .
$$

So $\lambda \in M(a, \mu)$ and we conclude that $M(a, \mu)$ is weak ${ }^{*}$-closed.

We claim that the family $\{M(a, \mu): a \in A, \mu$ a state $\}$ has the finite intersection property. If so, then as the unit ball of $A^{*}$ is weak $^{*}$-compact, there is $\lambda \in M(a, \mu)$ for all $a, \mu$. Set $M=\lambda \star \lambda$ so

$$
\langle M, a \cdot \mu\rangle=\langle\lambda, \lambda \cdot(a \cdot \mu)\rangle=\langle\lambda, \lambda \cdot a\rangle=\langle M, a\rangle,
$$

that is, $M$ is left invariant. As $\Phi$ is a unital $*$-homomorphism, and $\lambda$ is a state, also $M$ is a state as required to show (1).

To show the finite intersection property, we use induction. Let $a_{1}, \cdots, a_{n} \in A$ and $\mu_{1}, \cdots, \mu_{n}$ be states on $A$, and suppose that $\lambda \in \bigcap_{j=1}^{n-1} M\left(a_{j}, \mu_{j}\right)$. As (3) holds, we can find $\phi \in M\left(\lambda \cdot a_{n}, \mu_{n}\right)$, so

$$
0=\phi \cdot\left(\lambda \cdot a_{n}-\lambda \cdot a_{n} \cdot \mu_{n}\right)=(\phi \star \lambda) \cdot\left(a_{n}-a_{n} \cdot \mu_{n}\right) .
$$

However, for $1 \leq j<n$,

$$
(\phi \star \lambda) \cdot\left(a_{j}-a_{j} \cdot \mu_{j}\right)=\phi \cdot\left(\lambda \cdot\left(a_{j}-a_{j} \cdot \mu_{j}\right)\right)=\phi \cdot 0=0 .
$$

Thus $\phi \star \lambda \in \bigcap_{j=1}^{n} M\left(a_{j}, \mu_{j}\right)$, and so the result follows by induction.

Remark 4.5. If $M$ is a left invariant mean on $A$ then for any state $\mu \in A^{*}$ and $a \in A$, we have that $(M \star \mu) \cdot a=M \cdot(\mu \cdot a)=\langle M, \mu \cdot a\rangle 1=\langle M \star \mu, a\rangle 1$ and so $M \star \mu$ is also a left invariant mean. Thus, if $t 1 \in K(a)$, that is, there is a state $\mu$ with $\mu \cdot a=t 1$, then $(M \star \mu) \cdot a=M \cdot(\mu \cdot a)=t 1$. Thus $K(a) \cap \mathbb{C} 1=\{\lambda \cdot a: \lambda$ a left invariant mean $\}$.

Theorem 4.6. Let $(A, \Phi, V, H)$ be a $C^{*}$-Eberlein algebra with $A$ unital. Then $A$ has a left invariant mean. 
Proof. We verify condition (22) of the previous theorem. Indeed, we shall show that $K(a) \cap \mathbb{C} 1 \neq \emptyset$ for $a \in A_{V}$. This suffices, for if $a \in A$ there is a sequence $\left(a_{n}\right)$ in $A_{V}$ converging to $a$. By assumption, for each $n$ there is a state $\mu_{n}$ and $t_{n} \in \mathbb{C}$ with $\mu_{n} \cdot a_{n}=t_{n} 1$. Notice that $\left|t_{n}\right| \leq\left\|\mu_{n}\right\|\left\|a_{n}\right\|=\left\|a_{n}\right\| \rightarrow\|a\|$. So by moving to subnets, we may suppose that $t_{n} \rightarrow t \in \mathbb{C}$ and $\mu_{n} \rightarrow \mu$ weak* Then, for $\lambda \in A^{*}$,

$$
\begin{aligned}
\left|\left\langle\lambda, \mu_{n} \cdot a_{n}\right\rangle-\langle\lambda, \mu \cdot a\rangle\right| & =\left|\left\langle\mu_{n}, a_{n} \cdot \lambda\right\rangle-\langle\mu, a \cdot \lambda\rangle\right| \\
& \leq\left|\left\langle\mu_{n}, a_{n} \cdot \lambda\right\rangle-\left\langle\mu_{n}, a \cdot \lambda\right\rangle\right|+\left|\left\langle\mu_{n}, a \cdot \lambda\right\rangle-\langle\mu, a \cdot \lambda\rangle\right| \\
& =\left|\left\langle\mu_{n}, a_{n} \cdot \lambda-a \cdot \lambda\right\rangle\right|+\left|\left\langle\mu_{n}-\mu, a \cdot \lambda\right\rangle\right|,
\end{aligned}
$$

which converges to 0 , as $a_{n} \cdot \lambda-a \cdot \lambda \rightarrow 0$ in norm, and $\mu_{n}-\mu \rightarrow 0$ weak* . So $t_{n} 1=\mu_{n} \cdot a_{n} \rightarrow \mu \cdot a$ weakly, hence $\mu \cdot a=t 1$. So $K(a) \cap \mathbb{C} 1 \neq \emptyset$ for all $a$.

By replacing $V$ by $V_{12}$ acting on $H \otimes \ell^{2}$, we may suppose that $A_{V}=\left\{\left(\operatorname{id} \otimes \omega_{\xi, \eta}\right)(V): \xi, \eta \in H\right\}$. So let $a=\left(\mathrm{id} \otimes \omega_{\xi, \eta}\right)(V)$. As in the proof of Proposition 3.22, $\mu \cdot a=\left(\mathrm{id} \otimes V_{\mu} \omega_{\xi, \eta}\right)(V)=\left(\mathrm{id} \otimes \omega_{V_{\mu} \xi, \eta}\right)(V)$ for $\mu \in A^{*}$. As $V$ is unitary, by Proposition 4.2, if $\xi_{0}$ is the average of $\xi$, then $\xi_{0} \in \operatorname{Inv}(V)$ and $\xi_{0}$ is in the closure of $\left\{V_{\mu} \xi: \mu\right.$ a state $\}$. So there is a net of states $\left(\mu_{i}\right)$ with $V_{\mu_{i}} \xi \rightarrow \xi_{0}$ in norm. Passing to a subnet, we may suppose that $\mu_{i} \rightarrow \mu$ weak* So

$$
\mu \cdot a=\left(\operatorname{id} \otimes \omega_{V_{\mu} \xi, \eta}\right)(V)=\lim _{i}\left(\operatorname{id} \otimes \omega_{V_{\mu_{i}} \xi, \eta}\right)(V)=\left(\operatorname{id} \otimes \omega_{\xi_{0}, \eta}\right)(V)=\left(\xi_{0} \mid \eta\right) 1
$$

as $\xi_{0}$ is invariant. Hence $\left(\xi_{0} \mid \eta\right) 1 \in K(a)$, as required.

Theorem 4.7. Let $(A, \Phi, V, H)$ be a $C^{*}$-Eberlein algebra with $A$ unital. Let $M \in A^{*}$ be a left invariant mean. Then $M$ is the unique left invariant mean, and $(M \otimes \mathrm{id})(V) \in \mathcal{B}(H)$ is the orthogonal projection onto $\operatorname{Inv}(V)$. If $V$ is unitary, then $M$ is also right invariant.

Proof. Let $p=(M \otimes \mathrm{id})(V) \in \mathcal{B}(H)$. For $a=(\mathrm{id} \otimes \omega)(V) \in A$ we have that $\langle M, a\rangle 1=M \cdot a=(\mathrm{id} \otimes p \omega)(V)$. So with $\omega=\omega_{\xi, \eta}$ we see that for any $\mu \in A^{*}$,

$$
(p \xi \mid \eta)\langle\mu, 1\rangle=\langle M \otimes \omega, V\rangle\langle\mu, 1\rangle=\langle M, a\rangle\langle\mu, 1\rangle=\langle\mu,(\operatorname{id} \otimes p \omega)(V)\rangle=\left(V_{\mu} p \xi \mid \eta\right),
$$

so $V_{\mu} p \xi=\langle\mu, 1\rangle p \xi$ for all $\mu \in A^{*}$, that is, $p \xi \in \operatorname{Inv}(V)$.

Conversely, if $\xi \in \operatorname{Inv}(V)$ then $V_{\mu} \xi=\langle\mu, 1\rangle \xi$ for all $\xi \in A^{*}$, and so in particular, $p \xi=V_{M} \xi=\xi$ as $M$ is a state. So $p$ is an idempotent with image $\operatorname{Inv}(V)$. As $p$ is contractive, $p$ must be the orthogonal projection onto $\operatorname{Inv}(V)$.

If $N$ is also a left invariant mean then also $(N \otimes \mathrm{id})(V)=p$. So for $a=($ id $\otimes \omega)(V) \in A_{V}$ we find that $\langle N, a\rangle=\langle p, \omega\rangle=\langle M, a\rangle$. By density of $A_{V}$ in $A$, it follows that $M=N$.

That $p \xi \in \operatorname{Inv}(V)$ for all $\xi$ can be equivalently stated as $V(1 \otimes p)=1 \otimes p$. If $V$ is unitary then also $V^{*}(1 \otimes p)=1 \otimes p$ and so $(1 \otimes p) V=1 \otimes p$. Then, for $a=($ id $\otimes \omega)(V) \in A_{V}$, we see that

$$
a \cdot M=(\mathrm{id} \otimes \omega p)(V)=(\mathrm{id} \otimes \omega)((1 \otimes p) V)=(\mathrm{id} \otimes \omega)(1 \otimes p)=\langle M, a\rangle 1 .
$$

By continuity this holds for all $a \in A$ and so $M$ is right invariant.

Remark 4.8. Let $\sigma: A^{* *} \bar{\otimes} A^{* *} \rightarrow A^{* *} \bar{\otimes} A^{* *}$ be the swap map, so $\sigma \Phi$ induces the opposite multiplication on $A^{*}$. As $A_{V}$ is norm dense in $A$, which is a $C^{*}$-algebra, it follows that $A_{V^{*}}$ and $A_{V}$ have the same closure (which is $A$ ). Then $V^{*}$ will be a corepresentation for $\sigma \Phi$, and so we conclude that $\left(A, \sigma \Phi, V^{*}, H\right)$ will be a $C^{*}$-Eberlein algebra.

In this way, we can swap the roles of left and right invariant means. So the previous theorem also shows that right invariant means are unique, and if $V$ is unitary, right invariant means are invariant. 


\section{Compactifications}

We take a category theory approach to compactifications, as follows. By a "compactification" of $G \in$ LCG we mean the universal object in one of $\mathrm{CSCH}, \mathrm{CWCH}, \mathrm{CSTS}, \mathrm{CG}$. Letting Cat be one of these categories, this means that we seek $K \in$ Cat and a morphism $G \rightarrow K$ with the universal property that for any $H \in$ Cat and any morphism $G \rightarrow H$, there is a unique morphism $K \rightarrow H$ with the following diagram commuting:

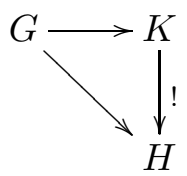

For example, as explained in [10, Section 2], one can think of the Bohr or almost periodic compactification of $G$ as being a universal object in CG, the category of compact groups. This is just the "largest" compact group to contain a dense homomorphic copy of our starting semigroup. Working instead in CSTS we obtain the weakly almost periodic compactification.

Let us think about what happens in $\mathrm{CWCH}$ in the context of [39]. Well, [39, Theorem 2.17] shows that for $G \in \mathrm{LCG}$ there is $G^{\mathcal{C H}} \in \mathrm{CWCH}$ and a morphism $\epsilon_{\mathcal{C H}}: G \rightarrow G^{\mathcal{C H}}$ with dense range, such that for any $S \in \mathrm{CWCH}$ with $\phi: G \rightarrow S$ having dense range, there is $\theta: G^{\mathcal{C} H} \rightarrow S$ with $\theta \circ \epsilon_{\mathcal{C} H}=\phi$; compare the definitions in [39, Section 2.1]. This is also a compactification in our sense: if $S \in \mathrm{CWCH}$ and $\phi: G \rightarrow S$ is any morphism, then set $S^{\prime}$ to be the closure of the image of $\phi$, so also $S^{\prime} \in \mathrm{CWCH}$. Let $\phi^{\prime}: G \rightarrow S^{\prime}$ be the corestriction of $\phi$. So there is $\theta^{\prime}: G^{\mathcal{C} H} \rightarrow S^{\prime}$, which is surjective, with $\theta^{\prime} \circ \epsilon^{\mathcal{C} H}=\phi^{\prime}$. Let $\theta$ be the composition of $\theta^{\prime}$ with the inclusion $S^{\prime} \rightarrow S$, so $\theta \circ \epsilon^{\mathcal{C} H}=\phi$. As $\epsilon^{\mathcal{C} H}$ has dense range, it is clear that $\theta$ is unique, as required.

We now wish to construct compactifications in our non-commutative setting, using the notion of morphisms considered in the previous section. Recall the notion of an embedded $C^{*}$-Eberlein algebra from Definition 3.29, Such $C^{*}$-Eberlein algebras biject with unitary corepresentations $U$ of $C_{0}^{u}(\mathbb{G})$ such that the norm closure of $\left\{(\mathrm{id} \otimes \omega)(U): \omega \in \mathcal{B}(H)_{*}\right\}$ is a $\mathrm{C}^{*}$-algebra, say $A$.

Theorem 5.1. Let $\mathbb{G}$ be a locally compact quantum group. There is an (essentially unique) unital embedded $C^{*}$-Eberlein algebra $\left(A, \Phi_{A}, U_{A}, H_{A}\right)$ such that for any other unital embedded $C^{*}$-Eberlein algebra $(B, \Phi, U, H)$, we have that $B \subseteq A$ with the inclusion $B \rightarrow A$ being a morphism.

Proof. We claim that it is enough to find an embedded $\mathrm{C}^{*}$-Eberlein $A$ such that any other embedded $\mathrm{C}^{*}$-Eberlein algebra $B$ is contained in $A$. Indeed, if so, then the inclusion $B \rightarrow A$ is non-degenerate, as it is unital. As both $A$ and $B$ are embedded, the products on $A^{*}$ and $B^{*}$ are induced by $\Delta_{u}$, and hence we immediately see that $A^{*} \rightarrow B^{*}$ is a homomorphism, so $B \rightarrow A$ is a morphism.

We now show the existence of $A$. Let

$$
X=\left\{(\mathrm{id} \otimes \omega)\left(U_{B}\right):\left(B, \Phi_{B}, U_{B}, H_{B}\right) \text { an embedded } \mathrm{C}^{*} \text {-Eberlein algebra }\right\},
$$

a subset of $M\left(C_{0}^{u}(\mathbb{G})\right)$. For each $x \in X$ let $U_{x}$ be a choice of suitable corepresentation on $H_{x}$, and $\omega_{x} \in \mathcal{B}\left(H_{x}\right)_{*}$, such that $x=\left(\mathrm{id} \otimes \omega_{x}\right)\left(U_{x}\right)$. Let $A$ be the $\mathrm{C}^{*}$-algebra generated by slices of $\left\{U_{x}: x \in X\right\}$, and let $U$ be the unitary corepresentation

$$
\bigoplus_{x \in X} U_{x} \oplus \bigoplus_{x \neq y} U_{x} \oplus U_{y} \oplus \bigoplus_{n \geq 3} \bigoplus_{x_{1} \neq x_{2} \neq \cdots \neq x_{n}} U_{x_{1}} \oplus \cdots \oplus U_{x_{n}}
$$

acting on $H$ say. This is a sort of "free-product" construction. By slicing $U$ we generate elements in $A$; for example, looking at the second term, we get elements (amoungst others) of the form (id $\left.\otimes \omega_{1}\right)\left(U_{x}\right)\left(\mathrm{id} \otimes \omega_{2}\right)\left(U_{y}\right)$ for $x \neq y$. It follows that slices of $U$ will be norm dense in $A$ and hence $A$ is an embedded $\mathrm{C}^{*}$-Eberlein algebra.

By construction, for any embedded $\mathrm{C}^{*}$-Eberlein algebra $\left(B, \Phi_{B}, U_{B}, H_{B}\right)$ and any $\omega \in \mathcal{B}\left(H_{B}\right)_{*}$, we have that $x=(\mathrm{id} \otimes \omega)\left(U_{B}\right) \in X$ and so in particular $x \in A$. As such $x$ are dense in $B$, it follows that $B \subseteq A$, as required. 
Let $E(\mathbb{G})=\left(\mathcal{E}(\mathbb{G}), \Phi_{\mathbb{G}}, U_{\mathbb{G}}, H_{\mathbb{G}}\right)$ be the embedded $C^{*}$-Eberlein algebra given by the previous theorem. Notice that $\mathcal{E}(\mathbb{G}), \Phi_{\mathbb{G}}$ are unique, but there is some, unimportant, choice in $U_{\mathbb{G}}, H_{\mathbb{G}}$. There is a further notational point: by definition, $U_{\mathbb{G}}$ is a "corepresentation" of $\mathcal{E}(\mathbb{G})$, and so $U_{\mathbb{G}} \in \mathcal{E}(\mathbb{G})^{* *} \bar{\otimes} \mathcal{B}\left(H_{\mathbb{G}}\right)$. The construction shows that $U_{\mathbb{G}}$ arises from Proposition 3.25 applied to a unitary corepresentation of $C_{0}^{u}(\mathbb{G})$. We shall abuse notation, and also write $U_{\mathbb{G}}$ for this unitary in $M\left(C_{0}^{u}(\mathbb{G}) \otimes \mathcal{B}_{0}\left(H_{\mathbb{G}}\right)\right)$.

Theorem 5.2. $E(\mathbb{G})$ is a compactification of $\mathbb{G}$ in the category of $C^{*}$-Eberlein algebras. That is, given any $C^{*}$-Eberlein algebra $\mathbb{S}=\left(B, \Phi_{B}, U_{B}, H_{B}\right)$ with $B$ unital, and any morphism $\mathbb{G} \rightarrow \mathbb{S}$, there is a unique morphism $E(\mathbb{G}) \rightarrow \mathbb{S}$ making the diagram commute:

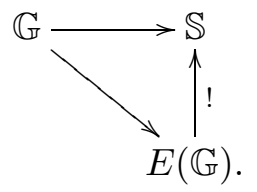

Proof. Let $\mathbb{G} \rightarrow \mathbb{S}$ be induced by $\theta: B \rightarrow M\left(C_{0}^{u}(\mathbb{G})\right)$. Then $\theta(B)$ is an embedded $C^{*}$-Eberlein algebra and so $\theta(B) \subseteq \mathcal{E}(\mathbb{G})$. Hence we may let $\theta_{0}$ be the corestriction of $\theta$ to a map $B \rightarrow \mathcal{E}(\mathbb{G})$. As a unital $*-$ homomorphism, this is non-degenerate, and is hence a morphism; clearly the resulting diagram commutes:

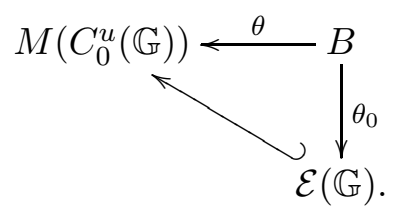

If $\phi: B \rightarrow \mathcal{E}(\mathbb{G})$ is another morphism making the diagram commute, then as the inclusion $j: \mathcal{E}(\mathbb{G}) \rightarrow$ $M\left(C_{0}^{u}(\mathbb{G})\right)$ is injective, we see that as $j \theta_{0}=\theta=j \phi$, also $\theta_{0}=\phi$. Hence $\theta_{0}$ is unique.

For any embedded $C^{*}$-Eberlein algebra, the generating corepresentation is unitary, and we can apply the results of Section 4 to show the following.

Theorem 5.3. For any $\mathbb{G}$ the $C^{*}$-Eberlein algebra $E(\mathbb{G})$ admits a unique invariant mean.

Thanks to Remark 3.21 , we know that $C_{0}^{u}(\mathbb{G}) \subseteq \mathcal{E}(\mathbb{G})$. The following is again known in the classical situation, for example [3, Corollary 3.7].

Proposition 5.4. Let $\mathbb{G}$ be a locally compact, non-compact, quantum group. Let $M$ be the unique invariant mean on $\mathcal{E}(\mathbb{G})$. Then $M$ annihilates $C_{0}^{u}(\mathbb{G})$.

Proof. Suppose not, so if $\varphi$ is $M$ restricted to $C_{0}^{u}(\mathbb{G})$, then $\varphi$ is a non-zero state. That $M$ is left-invariant, and that the inclusion $C_{0}^{u}(\mathbb{G}) \rightarrow \mathcal{E}(\mathbb{G})$ is a morphism, implies that $\mu \star \varphi=\varphi\langle\mu, 1\rangle$ for all $\mu \in C_{0}^{u}(\mathbb{G})^{*}$.

Now, by Cohen Factorisation, [29, Proposition A.2], there is $a \in C_{0}^{u}(\mathbb{G})$ and $\phi \in C_{0}^{u}(\mathbb{G})^{*}$ with $\varphi=\phi a$. For $x \in C_{0}^{u}(\mathbb{G})$ we know that $(1 \otimes a) \Delta(x) \in C_{0}^{u}(\mathbb{G}) \otimes C_{0}^{u}(\mathbb{G})$, see [23, Proposition 6.1], and so

$$
(\mathrm{id} \otimes \phi)((1 \otimes a) \Delta(x)) \in C_{0}^{u}(\mathbb{G}) .
$$

Then, for $\mu \in C_{0}^{u}(\mathbb{G})^{*}$, by left-invariance,

$$
\langle\mu, 1\rangle \varphi(x)=\langle\mu \star \varphi, x\rangle=\langle\mu \otimes \phi,(1 \otimes a) \Delta(x)\rangle .
$$

As $\mu$ was arbitrary, this implies that $\varphi(x) 1=(\mathrm{id} \otimes \phi)((1 \otimes a) \Delta(x)) \in C_{0}^{u}(\mathbb{G})$ and so by a suitable choice of $x$ we conclude that $1 \in C_{0}^{u}(\mathbb{G})$ and so $\mathbb{G}$ is compact. 


\section{The Quantum Bohr compactification}

For a locally compact group $G$, we know that wap $(G)$ splits as a direct sum of the almost periodic functions $\operatorname{ap}(G)$ and those $f \in \operatorname{wap}(G)$ such that $|f|^{2}$ is annihilated by the invariant mean; see [12, Theorem 5.11] or for a more semigroup-theoretic approach, see [4, Theorem 3.11, Chapter 4]. For earlier results about positive definite functions (that is, in our language, about the Eberlein compactification) were shown by Godement in [17, see also [13, Addenda, 16.5.2]. Over the next two sections, we develop analogous results for non-commutative $C^{*}$-Eberlein algebras, starting with an investigation of the quantum analogue of almost periodic functions.

In [36], Soltan explored a similar construction to that in the above section, but for compact quantum groups. We recall, [42, 28], that a compact quantum group is a unital $C^{*}$-bialgebra $(K, \Delta)$ with the "cancellation rules", namely that the following sets are both linearly dense in $A \otimes A$ :

$$
\{(a \otimes 1) \Delta(b): a, b \in K\}, \quad\{(1 \otimes a) \Delta(b): a, b \in K\} .
$$

Then, for any $C^{*}$-bialgebra $\left(A, \Delta_{A}\right)$ Soltan shows how to construct a compact quantum group $\left(K, \Delta_{K}\right)$ and a morphism (here meaning a non-degenerate $*$-homomorphism intertwining the coproducts) $K \rightarrow M(A)$ such that for any compact quantum group $\left(B, \Delta_{B}\right)$ and any morphism $B \rightarrow M(A)$, there is a morphism $B \rightarrow K$ making the three morphisms commute. Again, the construction realises $K$ as a subalgebra of $M(A)$ and $\Delta_{K}$ as the restriction of $\Delta_{A}$.

Let $U=\left(U_{i j}\right) \in \mathbb{M}_{n}(M(A))=M\left(A \otimes \mathbb{M}_{n}\right)$ be a finite-dimensional (unitary, or just invertible) corepresentation of $\left(A, \Delta_{A}\right)$. We say that $U$ is admissible if the "transpose" $\left(U_{j i}\right)$ is also invertible in the algebra $\mathbb{M}_{n}(M(A))$. Then we can construct $K$ as the closed linear span of elements of the form $U_{i j}$ as $U$ varies over all admissible corepresentations.

One of the problems of Soltan's construction, further explored in [10], is that it might be hard to decide if a given finite-dimensional unitary corepresentation is admissible (although we note that there are no known counter-examples to the conjecture that any finite-dimensional unitary corepresentation of a locally compact quantum group is already admissible.) The following results are interesting from this perspective.

The second named author explored this construction more in [10]. In particular, we introduced the notation that, when Soltan's construction is applied to $C_{0}^{u}(\mathbb{G})$, we get the compact quantum group $\left(\mathbb{A P}(\mathbb{G}), \Delta_{\mathbb{A P}(\mathbb{G})}\right)$. So $\mathbb{A P}(\mathbb{G}) \subseteq M\left(C_{0}^{u}(\mathbb{G})\right)$ is the "maximal" compact quantum group inside $M\left(C_{0}^{u}(\mathbb{G})\right)$. We remark that if we work instead with $C_{0}(\mathbb{G})$, we may obtain a (possibly) different compact quantum group, but the underlying Hopf $*$-algebra is the same.

Proposition 6.1. For any $\mathbb{G}$ we have that $\mathbb{A P}(\mathbb{G}) \subseteq \mathcal{E}(\mathbb{G})$, and the mean on $\mathcal{E}(\mathbb{G})$ restricts to the Haar state on $\mathbb{A P}(\mathbb{G})$.

Proof. Set $A=\mathbb{A P}(\mathbb{G}) \subseteq M\left(C_{0}^{u}(\mathbb{G})\right)$. Let $W \in M\left(A \otimes \mathcal{B}_{0}(H)\right)$ be the left-regular representation for the compact quantum group $A$. Thus slices of $W$ are norm dense in $A$, and it is easy to verify that $\left(A,\left.\Delta_{u}\right|_{A}, W, H\right)$ is a $C^{*}$-Eberlein algebra. So we immediately see that $A \subseteq \mathcal{E}(\mathbb{G})$. The restriction of the mean on $\mathcal{E}(\mathbb{G})$ to $\mathbb{A P}(\mathbb{G})$ will be a bi-invariant state, and hence by uniqueness, is the Haar state.

Proposition 6.2. Let $V$ be a finite-dimensional sub-corepresentation of $U_{\mathbb{G}}$. Then $V$ is admissible.

Proof. This is similar to the argument for compact quantum groups, compare for example [28, Proposition 6.10], and so we shall not give all the details. By assumption, there is a finite-dimensional subspace $K \subseteq H_{\mathbb{G}}$ such that, with $q: H_{\mathbb{G}} \rightarrow K$ the orthogonal projection, we have that $U_{\mathbb{G}}(1 \otimes q)=(1 \otimes q) U_{\mathbb{G}}$ and $V=(1 \otimes q) U_{\mathbb{G}}(1 \otimes q) \in M\left(C_{0}^{u}(\mathbb{G}) \otimes \mathcal{B}(K)\right)$. If we fix an orthonormal basis $\left(e_{i}\right)_{i=1}^{n}$ for $K$, then we can view $V$ as a matrix $\left(V_{i j}\right) \in \mathbb{M}_{n}\left(M\left(C_{0}^{u}(\mathbb{G})\right)\right)$. Then $V$ is admissible if $\left(V_{j i}\right)$ is invertible, or equivalently, if $\bar{V}=\left(V_{i j}^{*}\right)$ is invertible. We shall show that actually $\bar{V}$ is similar to a unitary.

As $V$ is finite-dimensional and unitary, $V$ is the direct-sum of finitely many irreducible unitary corepresentations. So it suffices to prove the claim when $V$ is irreducible, which we now assume. As shown in 
28, Lemma 6.9], for example, $\bar{V}$ will also be irreducible. As $($ id $\otimes \omega)(V) \in \mathcal{E}(\mathbb{G})$ for all $\omega \in \mathcal{B}(K)_{*}$, we can apply Proposition 3.25 to form $V^{\prime} \in \mathcal{E}(\mathbb{G})^{* *} \bar{\otimes} \mathcal{B}(K)$ (actually, as $K$ is finite-dimensional, it is both easy to construct $V^{\prime}$, and we find that actually $V^{\prime} \in \mathcal{E}(\mathbb{G}) \otimes \mathcal{B}(K)$ ). Similarly let $U_{\mathbb{G}}^{\prime}$ be the copy of $U_{\mathbb{G}}$ in $\mathcal{E}(\mathbb{G})^{* *} \bar{\otimes} \mathcal{B}\left(H_{\mathbb{G}}\right)$. It is easy to verify that $V^{\prime}=(1 \otimes q) U_{\mathbb{G}}^{\prime}(1 \otimes q)$.

Let $M \in \mathcal{E}(\mathbb{G})^{*}$ be the invariant mean so, as after Definition 4.3, we have that $(M \otimes \mathrm{id}) \tilde{\Phi}_{\mathbb{G}}(x)=\langle x, M\rangle 1$ for $x \in \mathcal{E}(\mathbb{G})^{* *}$. We have the isomorphism

$$
\mathcal{B}\left(H_{\mathbb{G}}\right) \cong\left(\begin{array}{cc}
\mathcal{B}(K) & \mathcal{B}\left(K^{\perp}, K\right) \\
\mathcal{B}\left(K, K^{\perp}\right) & \mathcal{B}\left(K^{\perp}\right)
\end{array}\right)
$$

and similarly we can consider $\mathcal{E}(\mathbb{G})^{* *} \bar{\otimes} \mathcal{B}\left(H_{\mathbb{G}}\right)$ as a matrix algebra. Let $x \in \mathcal{B}\left(K, H_{\mathbb{G}}\right)$, and consider $1 \otimes x \in \mathcal{E}(\mathbb{G})^{* *} \bar{\otimes} \mathcal{B}\left(K, H_{\mathbb{G}}\right)$ regarded as the "left column" of our matrix algebra. This viewpoint allows us to define

$$
y=(M \otimes \mathrm{id})\left(U_{\mathbb{G}}^{\prime *}(1 \otimes x) \bar{V}^{\prime}\right) \in \mathcal{B}\left(K, H_{\mathbb{G}}\right) .
$$

As $\Phi$ is a $*$-homomorphism, it is easy to see that $\bar{V}$ will also be a corepresentation, and so

$$
\left(\tilde{\Phi}_{\mathbb{G}} \otimes \mathrm{id}\right)\left(U_{\mathbb{G}}^{\prime *}(1 \otimes x) \bar{V}^{\prime}\right)=U_{\mathbb{G} 23}^{\prime *} U_{\mathbb{G} 13}^{\prime *}(1 \otimes x) \bar{V}_{13}^{\prime} \bar{V}_{23}^{\prime} .
$$

Applying $(M \otimes$ id $\otimes$ id $)$ we find that

$$
1 \otimes y=1 \otimes(M \otimes \mathrm{id})\left(U_{\mathbb{G}}^{\prime *}(1 \otimes x) \bar{V}^{\prime}\right)=U_{\mathbb{G}}^{\prime *}(1 \otimes y) \bar{V}^{\prime}
$$

Thus $U_{\mathbb{G}}^{\prime}(1 \otimes y)=(1 \otimes y) \bar{V}^{\prime}$. As $\bar{V}^{\prime}$ is irreducible, if $y \neq 0$, in a standard way this shows that $\bar{V}^{\prime}$ is equivalent to a sub-corepresentation of $U_{\mathbb{G}}^{\prime}$, and hence is similar to a unitary, showing the same for $\bar{V}$ as required.

So we need to show that for some choice of $x$, we get a non-zero $y$. Towards a contradiction suppose not, so $y=0$ for all choices of $x$. For $\xi \in H_{\mathbb{G}}, \eta \in K$ let $x=\theta_{\xi, \eta}$ a rank-one operator $K \rightarrow H_{\mathbb{G}}$. Then, for $\alpha \in K, \beta \in H_{\mathbb{G}}$

$$
0=(y \alpha \mid \beta)=\left\langle U_{\mathbb{G}}^{\prime *}\left(1 \otimes \theta_{\xi, \eta}\right) \bar{V}^{\prime}, M \otimes \omega_{\alpha, \beta}\right\rangle=\left\langle M,\left(\mathrm{id} \otimes \omega_{\xi, \beta}\right)\left(U_{\mathbb{G}}^{\prime}\right)\left(\mathrm{id} \otimes \omega_{\alpha, \eta}\right)\left(\bar{V}^{\prime}\right)\right\rangle .
$$

Now, elements of the form $\left(\mathrm{id} \otimes \omega_{\xi, \beta}\right)\left(U_{\mathbb{G}}^{\prime}\right)$ are linearly dense in $\mathcal{E}(\mathbb{G})$, and (id $\left.\otimes \omega_{\alpha, \eta}\right)\left(\bar{V}^{\prime}\right)=\left(\mathrm{id} \otimes \omega_{\alpha, \eta}\right)\left(V^{\prime}\right)$ is a linear combination of the elements $V_{i j}^{*} \in \mathcal{E}(\mathbb{G})$. Thus $\left\langle M, a V_{i j}^{*}\right\rangle=0$ for all $a \in \mathcal{E}(\mathbb{G})$, in particular, $\left\langle M, V_{i j} V_{i j}^{*}\right\rangle=0$. As $\langle M, 1\rangle=1$ and $V$ is unitary, this leads to the required contradiction.

\subsection{Compact vectors}

In this section, we generalise the classical notion of a compact vector for a unitary group representation. Classically, see [5, Definition 1.7, Lemma 1.8] if $\pi$ is a unitary representation of a locally compact group $G$ on a Hilbert space $H$, then $\xi \in H$ is compact if and only if $\{\pi(g) \xi: g \in G\}$ is relatively compact in $H$, if and only if $g \mapsto(\pi(g) \xi \mid \xi)$ is an almost periodic function. The following is hence a slightly stronger definition, but it is appropriate for our needs.

Definition 6.3. Let $\mathbb{G}$ be a locally compact group, and let $U \in M\left(C_{0}^{u}(\mathbb{G}) \otimes \mathcal{B}_{0}(H)\right)$ be a unitary corepresentation. Define $\xi \in H$ to be a compact vector if

$$
\left(\mathrm{id} \otimes \omega_{\xi, \eta}\right)(U) \in \mathbb{A} \mathbb{P}\left(C_{0}^{u}(\mathbb{G})\right) \quad(\eta \in H) .
$$

It is easy to see that the collection of all compact vectors forms a closed subspace of $H$, say $H_{c}$.

From now on, fix a unitary corepresentation $U$ and let $H_{c}$ be the subspace of compact vectors. 
Lemma 6.4. The corepresentation $U$ restricts to $H_{c}$ forming a corepresentation $V \in M\left(C_{0}^{u}(\mathbb{G}) \otimes \mathcal{B}_{0}\left(H_{c}\right)\right)$ say. As $U$ is unitary, $U$ also restricts to $H_{c}^{\perp}$ forming a corepresentation $V_{0}$ say. Then $V_{0}$ has no non-zero compact vectors.

Proof. We wish to show that for $\mu \in C_{0}^{u}(\mathbb{G})^{*}$ and $\xi \in H_{c}$, we have that $(\mu \otimes \mathrm{id})(U) \xi \in H_{c}$. Set $U_{\mu}=(\mu \otimes \mathrm{id})(U)$, so for $\eta \in H$, we see that

$$
\left(\mathrm{id} \otimes \omega_{U_{\mu} \xi, \eta}\right)(U)=\left(\mathrm{id} \otimes \omega_{\xi, \eta}\right)\left(U\left(1 \otimes U_{\mu}\right)\right)=\left(\mathrm{id} \otimes \mu \otimes \omega_{\xi, \eta}\right)\left(U_{13} U_{23}\right)=(\mathrm{id} \otimes \mu) \Delta\left(\left(\mathrm{id} \otimes \omega_{\xi, \eta}\right)(U)\right) .
$$

As $\left(\operatorname{id} \otimes \omega_{\xi, \eta}\right)(U) \in \mathbb{A}\left(C_{0}^{u}(G)\right)$ we see that

$$
\Delta\left(\left(\mathrm{id} \otimes \omega_{\xi, \eta}\right)(U)\right) \in \mathbb{A} \mathbb{P}\left(C_{0}^{u}(G)\right) \otimes \mathbb{A P}\left(C_{0}^{u}(G)\right)
$$

the minimal tensor product, and so slices are also in $\mathbb{A P}\left(C_{0}^{u}(G)\right)$ as required to show that $U_{\mu} \xi \in H_{c}$ as claimed.

We next show that $V_{0}$ has no non-zero compact vectors. Indeed, if $\xi \in H_{c}^{\perp}$ is non-zero then $\xi$ is not compact for $U$, and so there is $\eta=\eta_{c}+\eta_{0} \in H_{c} \oplus H_{c}^{\perp}$ with $\left(\operatorname{id} \otimes \omega_{\xi, \eta}\right)(U) \notin \mathbb{A} \mathbb{P}\left(C_{0}^{u}(\mathbb{G})\right)$. As $\xi \in H_{c}^{\perp}$ and $H_{c}^{\perp}$ is invariant for $U$, it follows that

$$
\left(\mathrm{id} \otimes \omega_{\xi, \eta}\right)(U)=\left(\mathrm{id} \otimes \omega_{\xi, \eta_{0}}\right)(U)=\left(\mathrm{id} \otimes \omega_{\xi, \eta_{0}}\right)\left(V_{0}\right),
$$

which shows that $\xi$ is not compact for $V_{0}$, as required.

We shall now proceed to show that $V$ can be "identified" with a unitary corepresentation of $\mathbb{A P}\left(C_{0}^{u}(G)\right)$; part of our effort will be to make this statement precise.

At this point, we shall need a little more of the theory of (locally) compact quantum groups, which we now sketch. For a compact quantum group $A$, let $\varphi$ be the Haar state for $A$, with GNS space $H$ and representation $\pi: A \rightarrow \mathcal{B}(H)$. Then, for example using the regular corepresentation of $A$ on $H$, we can show that $\pi(A)$ also admits a coproduct making $\pi$ a morphism. We call $\pi(A)$ the reduced form of $A$, and with $\varphi$ as the left and right Haar weights, this becomes a locally compact quantum group in the sense of [25]. Let $\mathbb{K}$ be the locally compact quantum group thus induced, so $\pi(A)=C(\mathbb{K})$ and $H=L^{2}(\mathbb{K})$. Let $L^{\infty}(\mathbb{G})$ be the von Neumann algebra generated by $C(\mathbb{K})$ in $\mathcal{B}\left(L^{2}(\mathbb{K})\right)$; again an argument using the regular representation shows that $L^{\infty}(\mathbb{G})$ can be given a weak*-continuous coproduct which extends that on $C(\mathbb{K})$. Let $L^{1}(\mathbb{K})$ be the predual of $L^{\infty}(\mathbb{G})$ (or, equivalently, the collection of normal functionals on $C(\mathbb{K}))$ which becomes a Banach algebra for the pre-adjoint of the coproduct on $L^{\infty}(\mathbb{K})$. See, for example, [25, Section 8].

Let $\mathbb{G}$ be a (locally) compact quantum group. As the antipode $S$ is in general unbounded, the natural way to define a $*$-structure on $L^{1}(\mathbb{G})$ fails. Instead, we let $L_{\sharp}^{1}(\mathbb{G})$ be the collection of $\omega \in L^{1}(\mathbb{G})$ such that there is $\omega^{\sharp} \in L^{1}(\mathbb{G})$ with

$$
\left\langle x, \omega^{\sharp}\right\rangle=\overline{\left\langle S(x)^{*}, \omega\right\rangle} \quad\left(x \in D(S) \subseteq L^{\infty}(\mathbb{G})\right) .
$$

As $S\left(S(x)^{*}\right)^{*}=x$ for $x \in D(S)$, it follows that $\sharp$ gives an involution on $L_{\sharp}^{1}(\mathbb{G})$. Under the norm $\|\omega\|_{\sharp}=\max \left(\|\omega\|,\left\|\omega^{\sharp}\right\|\right)$ also $L_{\sharp}^{1}(\mathbb{G})$ becomes a Banach algebra. The left-regular representation

$$
\lambda: L^{1}(\mathbb{G}) \rightarrow C_{0}(\widehat{\mathbb{G}}) ; \quad \omega \mapsto(\omega \otimes \mathrm{id})(W)
$$

is a homomorphism with range dense in $C_{0}(\widehat{\mathbb{G}})$, and it restricts to a $*$-homomorphism on $L_{\sharp}^{1}(\mathbb{G})$. Indeed, $\omega \in L_{\sharp}^{1}(\mathbb{G})$ if and only if there is $\tau \in L^{1}(\mathbb{G})$ with $\lambda(\tau)=\lambda(\omega)^{*}$, and then $\tau=\omega^{\sharp}$. A "smearing" argument using the scaling group shows that $L_{\sharp}^{1}(\mathbb{G})$ is norm dense in $L^{1}(\mathbb{G})$; see [23, Section 3] and [24, Definition 2.3] and the discussion after.

Let $\mathbb{K}$ be the compact quantum group induced by $\mathbb{A P}\left(C_{0}^{u}(\mathbb{G})\right)$, so that $C(\mathbb{K})$ is the reduced form of $\mathbb{A P}\left(C_{0}^{u}(\mathbb{G})\right.$ ), and we have the quotient map $\phi: \mathbb{A P}\left(C_{0}^{u}(\mathbb{G})\right) \rightarrow C(\mathbb{K})$ (as shown in [10] this map can indeed 
fail to be an isomorphism). We can then restrict $\phi^{*}: C(\mathbb{K})^{*} \rightarrow \mathbb{A P}\left(C_{0}^{u}(G)\right)^{*}$, which is an isometric algebra homomorphism, to $L^{1}(\mathbb{K})$, still denoted by $\phi^{*}$. For $\omega \in L^{1}(\mathbb{K})$ let $\mu \in M\left(C_{0}^{u}(\mathbb{G})\right)^{*}$ be a Hahn-Banach extension of $\phi^{*}(\omega)$, recalling that $\mathbb{A P}\left(C_{0}^{u}(G)\right) \subseteq M\left(C_{0}^{u}(\mathbb{G})\right)$. Then define $(\mu \otimes \mathrm{id})(V) \in \mathcal{B}\left(H_{c}\right)$ in the usual way:

$$
((\mu \otimes \mathrm{id})(V) \xi \mid \eta)=\left\langle\mu,\left(\mathrm{id} \otimes \omega_{\xi, \eta}\right)(V)\right\rangle \quad\left(\xi, \eta \in H_{c}\right) .
$$

As (id $\left.\otimes \omega_{\xi, \eta}\right)(V) \in \mathbb{A} \mathbb{P}\left(C_{0}^{u}(G)\right)$ we hence see that $(\mu \otimes$ id $)(V)$ depends only on $\omega$ and not on the particular extension chosen. Hence define this to be $\pi(\omega) \in \mathcal{B}\left(H_{c}\right)$.

Lemma 6.5. The map $\pi: L^{1}(\mathbb{K}) \rightarrow \mathcal{B}\left(H_{c}\right)$ is a homomorphism, and when restricted to $L_{\sharp}^{1}(\mathbb{K})$ is a *-homomorphism.

Proof. Let $\omega \in L^{1}(\mathbb{K})$ and let $\mu \in M\left(C_{0}^{u}(\mathbb{G})\right)^{*}$ be an extension of $\phi^{*}(\omega)$. Treating $M\left(C_{0}^{u}(\mathbb{G})\right)$ as a subalgebra of $C_{0}^{u}(\mathbb{G})^{* *}$ we can further extend $\mu$ and then find a bounded net $\left(\mu_{\alpha}\right)$ in $C_{0}^{u}(\mathbb{G})^{*}$ with

$$
\lim _{\alpha}\left\langle\mu_{\alpha}, x\right\rangle=\langle\phi(x), \omega\rangle \quad\left(x \in \mathbb{A}\left(C_{0}(\mathbb{G})\right)\right) .
$$

It then follows that

$$
(\pi(\omega) \xi \mid \eta)=\left\langle\phi\left(\left(\operatorname{id} \otimes \omega_{\xi, \eta}\right)(V)\right), \omega\right\rangle=\lim _{\alpha}\left(\left(\mu_{\alpha} \otimes \mathrm{id}\right)(V) \xi \mid \eta\right) \quad\left(\xi, \eta \in H_{c}\right) .
$$

For $i=1,2$, let $\omega_{i} \in L^{1}(\mathbb{K})$ and choose a bounded net $\left(\mu_{\alpha}^{(i)}\right)$ as above. Then, for $\xi, \eta \in H_{c}$,

$$
\begin{aligned}
\left(\pi\left(\omega_{1}\right) \pi\left(\omega_{2}\right) \xi \mid \eta\right) & =\lim _{\alpha}\left(\left(\mu_{\alpha}^{(1)} \otimes \mathrm{id}\right)(V) \pi\left(\omega_{2}\right) \xi \mid \eta\right)=\lim _{\alpha} \lim _{\beta}\left(\left(\mu_{\alpha}^{(1)} \otimes \mathrm{id}\right)(V)\left(\mu_{\beta}^{(2)} \otimes \mathrm{id}\right)(V) \xi \mid \eta\right) \\
& =\lim _{\alpha} \lim _{\beta}\left\langle\mu_{\alpha}^{(1)} \otimes \mu_{\beta}^{(2)} \otimes \omega_{\xi, \eta}, V_{13} V_{23}\right\rangle=\lim _{\alpha} \lim _{\beta}\left\langle\mu_{\alpha}^{(1)} \star \mu_{\beta}^{(2)} \otimes \omega_{\xi, \eta}, V\right\rangle,
\end{aligned}
$$

as $V$ is a corepresentation. Now let $x \in \mathbb{A}\left(C_{0}^{u}(\mathbb{G})\right)$. As $\Delta(x) \in \mathbb{A P}\left(C_{0}^{u}(\mathbb{G})\right) \otimes \mathbb{A P}\left(C_{0}^{u}(\mathbb{G})\right)$ the minimal tensor product, we see that

$$
\lim _{\alpha}\left(\mu_{\alpha}^{(1)} \otimes \mathrm{id}\right) \Delta(x)=\left(\phi^{*}\left(\omega_{1}\right) \otimes \mathrm{id}\right) \Delta(x),
$$

with convergence in norm. Thus

$$
\begin{aligned}
\lim _{\alpha} \lim _{\beta}\left\langle\mu_{\alpha}^{(1)} \star \mu_{\beta}^{(2)}, x\right\rangle & =\lim _{\alpha} \lim _{\beta}\left\langle\mu_{\beta}^{(2)},\left(\mu_{\alpha}^{(1)} \otimes \mathrm{id}\right) \Delta(x)\right\rangle=\lim _{\alpha}\left\langle\phi^{*}\left(\omega_{2}\right),\left(\mu_{\alpha}^{(1)} \otimes \mathrm{id}\right) \Delta(x)\right\rangle \\
& =\left\langle\phi^{*}\left(\omega_{2}\right),\left(\phi^{*}\left(\omega_{1}\right) \otimes \mathrm{id}\right) \Delta(x)\right\rangle=\left\langle\phi^{*}\left(\omega_{1}\right) \star \phi^{*}\left(\omega_{2}\right), x\right\rangle=\left\langle\phi^{*}\left(\omega_{1} \star \omega_{2}\right), x\right\rangle .
\end{aligned}
$$

Combining this with the above, we see that

$$
\left(\pi\left(\omega_{1}\right) \pi\left(\omega_{2}\right) \xi \mid \eta\right)=\left\langle\phi^{*}\left(\omega_{1} \star \omega_{2}\right),\left(\mathrm{id} \otimes \omega_{\xi, \eta}\right)(V)\right\rangle=\left(\pi\left(\omega_{1} \star \omega_{2}\right) \xi \mid \eta\right) .
$$

So $\pi$ is a homomorphism.

As a remark, it would have been tempting to work more directly with $\mu_{i} \in M\left(C_{0}(\mathbb{G})\right)^{*}$, but then it's not clear what meaning $\mu_{1} \star \mu_{2}$ has; the above argument is essentially a resolution of this problem.

We now show that $\pi$ is a $*$-homomorphism on $L_{\sharp}^{1}(\mathbb{G})$. We know that $\phi \circ S_{\mathbb{A P}} \subseteq S_{\mathbb{K}} \circ \pi$, see $[23$, Remark 12.1]. A similar remark applies to the inclusion $\mathbb{A P}\left(C_{0}(\mathbb{K})\right) \subseteq M\left(C_{0}(\mathbb{G})\right)$ which is a morphism. So for each $\omega \in \mathcal{B}\left(H_{c}\right)_{*}$ we have that $(\mathrm{id} \otimes \omega)(V) \in D\left(S_{\mathbb{A P}}\right)$ and $S_{\mathbb{A P}}((\mathrm{id} \otimes \omega)(V))=(\mathrm{id} \otimes \omega)\left(V^{*}\right)$ and hence also $\phi((\operatorname{id} \otimes \omega)(V)) \in D\left(S_{\mathbb{K}}\right)$ and $S_{\mathbb{K}}(\phi((\operatorname{id} \otimes \omega)(V)))=\phi\left((\operatorname{id} \otimes \omega)\left(V^{*}\right)\right)$. So, for $\omega \in L_{\sharp}^{1}(\mathbb{K})$,

$$
\begin{aligned}
\left(\pi\left(\omega^{\sharp}\right) \xi \mid \eta\right) & =\left\langle\phi\left(\left(\operatorname{id} \otimes \omega_{\xi, \eta}\right)(V)\right), \omega^{\sharp}\right\rangle=\overline{\left\langle S_{\mathbb{K}}\left(\phi\left(\left(\operatorname{id} \otimes \omega_{\xi, \eta}\right)(V)\right)\right)^{*}, \omega\right\rangle}=\overline{\left\langle\phi\left(\left(\operatorname{id} \otimes \omega_{\eta, \xi}\right)(V)\right), \omega\right\rangle} \\
& =\overline{(\pi(\omega) \eta \mid \xi)}=\left(\pi(\omega)^{*} \xi \mid \eta\right),
\end{aligned}
$$

as required. 
It is not clear to us that $\pi$ is non-degenerate, but see Corollary 6.8 below. However, let $H_{c c}$ be the closed linear span of $\left\{\pi(\omega) \xi: \xi \in H_{c}, \omega \in L^{1}(\mathbb{K})\right\}$, and note that if we replace $L^{1}(\mathbb{K})$ by $L_{\sharp}^{1}(\mathbb{K})$ we get the same closure. Then, for $\eta \in H_{c c}^{\perp}$ we find that

$$
(\pi(\omega) \eta \mid \xi)=\left(\eta \mid \pi\left(\omega^{\sharp}\right) \xi\right)=0 \quad\left(\xi \in H_{c}, \omega \in L_{\sharp}^{1}(\mathbb{K})\right),
$$

and so $\pi$ restricts to the zero representation on $H_{c c}^{\perp}$. As products are dense in $L^{1}(\mathbb{K})$ it follows that the closed linear span of $\left\{\pi(\omega) \xi: \omega \in L^{1}(\mathbb{K}), \xi \in H_{c c}\right\}$ equals $H_{c c}$ again, and so $\pi$, restricted to $H_{c c}$, is non-degenerate, say giving $\pi_{c c}$.

Proposition 6.6. There is a unitary corepresentation $Y$ of $\mathbb{A P}\left(C_{0}^{u}(\mathbb{G})\right)$ on $H_{c c}$ such that, if we zero-extend $Y$ to a degenerate representation on $H_{c}$, then $(\mathrm{id} \otimes \omega)(Y)=(\mathrm{id} \otimes \omega)(V) \in M\left(C_{0}^{u}(\mathbb{G})\right)$ for all $\omega \in \mathcal{B}\left(H_{c}\right)_{*}$.

Proof. As $\pi_{c c}$ is a non-degenerate $*$-homomorphism of $L_{\sharp}^{1}(\mathbb{K})$, by [23, Corollary 4.3], there is a unitary corepresentation $X \in M\left(C(\mathbb{K}) \otimes \mathcal{B}_{0}\left(H_{c c}\right)\right)$ with $(\omega \otimes \mathrm{id})(X)=\pi_{c c}(\omega)$ for $\omega \in L^{1}(\mathbb{K})$. Treating $\mathcal{B}\left(H_{c c}\right)$ as a "corner" of $\mathcal{B}\left(H_{c}\right)$, we may "zero-extend" $X$ to a member of $M\left(C(\mathbb{K}) \otimes \mathcal{B}_{0}\left(H_{c}\right)\right)$. A careful check shows that $\pi(\omega)=(\omega \otimes \mathrm{id})(X)$ for all $\omega \in L^{1}(\mathbb{K})$. Then, for $\tau \in \mathcal{B}\left(H_{c}\right)_{*}$, we have that

$$
\langle(\operatorname{id} \otimes \tau)(X), \omega\rangle=\langle\pi(\omega), \tau\rangle=\left\langle\phi^{*}(\omega),(\operatorname{id} \otimes \tau)(V)\right\rangle=\langle\phi((\operatorname{id} \otimes \tau)(V)), \omega\rangle \quad\left(\omega \in L^{1}(\mathbb{K})\right) .
$$

So $($ id $\otimes \tau)(X)=\phi(($ id $\otimes \tau)(V))$.

Using [23, Proposition 6.6] we may "lift" $X \in M\left(C(\mathbb{K}) \otimes \mathcal{B}_{0}\left(H_{c c}\right)\right)$ to a unique unitary corepresentation $X_{u} \in M\left(C^{u}(\mathbb{K}) \otimes \mathcal{B}_{0}\left(H_{c c}\right)\right.$ ), and then push this down to a (unique) unitary corepresentation $Y \in M\left(\mathbb{A P}\left(C_{0}^{u}(\mathbb{G})\right) \otimes \mathcal{B}_{0}\left(H_{c c}\right)\right)$ with $(\phi \otimes \mathrm{id})(Y)=X$. Let $W \in M\left(\mathbb{A P}\left(C_{0}^{u}(\mathbb{G})\right) \otimes L^{2}(\mathbb{K})\right)$ be the left regular representation of $\mathbb{A P}\left(C_{0}^{u}(\mathbb{G})\right)$, so that

$$
W^{*}(1 \otimes \phi(x)) W=(\mathrm{id} \otimes \phi) \Delta_{\mathbb{A}}(x) \quad\left(x \in \mathbb{A P}\left(C_{0}^{u}(\mathbb{G})\right)\right) .
$$

(This follows from equation (5.10) in [42], or from [23], compare the proof of [10, Lemma 3.6]). As $Y$ is a corepresentation, it follows that

$$
W_{12}^{*}(\phi \otimes \mathrm{id})(Y)_{23} W_{12}=(\mathrm{id} \otimes \phi \otimes \mathrm{id})\left(Y_{13} Y_{23}\right) \Longrightarrow Y_{13}=W_{12}^{*} X_{23} W_{12} X_{23}^{*},
$$

as $(\phi \otimes \mathrm{id})(Y)=X$. This of course being the proof that $Y$ is uniquely determined by $X$. If we now zero-extend $Y$ to a member of $M\left(\mathbb{A P}\left(C_{0}(\mathbb{G})\right) \otimes \mathcal{B}_{0}\left(H_{c}\right)\right)$, then again $W_{12}^{*} X_{23} W_{12}=Y_{13} X_{23}$ and so $W_{12}^{*} X_{23} W_{12} X_{23}^{*}=Y_{13}(1 \otimes 1 \otimes p)=Y_{13}$ as $Y_{13}$ is zero on $H_{c c}^{\perp}$ by construction, where here $p: H_{c} \rightarrow H_{c c}$ is the orthogonal projection.

Now let $\xi, \eta \in H_{c}$, so

$$
\left(\mathrm{id} \otimes \omega_{\xi, \eta}\right)(Y) \otimes 1=\left(\mathrm{id} \otimes \mathrm{id} \otimes \omega_{\xi, \eta}\right)\left(W_{12}^{*} X_{23} W_{12} X_{23}^{*}\right)=W^{*} \sum_{i}\left(\mathrm{id} \otimes \mathrm{id} \otimes \omega_{\xi, \eta}\right)\left(X_{23}\left(W \otimes \theta_{e_{i}, e_{i}}\right) X_{23}^{*}\right),
$$

where $\left(e_{i}\right)$ is an orthonormal basis for $H_{c}$ and $\theta_{e_{i}, e_{i}}$ is the rank-one projection onto the span of $e_{i}$. Notice that $\sum_{i}\left(W \otimes \theta_{e_{i}, e_{i}}\right)$ converges strictly to $W \otimes 1$ in $M\left(\mathbb{A P}\left(C_{0}^{u}(\mathbb{G})\right) \otimes \mathcal{B}_{0}\left(L^{2}(\mathbb{K})\right) \otimes \mathcal{B}_{0}\left(H_{c c}\right)\right)$, and as slice maps are strictly continuous, the sum above converges strictly in $M\left(\mathbb{P}\left(C_{0}^{u}(\mathbb{G})\right) \otimes C_{0}(\mathbb{K})\right)$. Thus

$$
\left(\mathrm{id} \otimes \omega_{\xi, \eta}\right)(Y) \otimes 1=W^{*} \sum_{i}\left(1 \otimes\left(\mathrm{id} \otimes \omega_{e_{i}, \eta}\right)(X)\right) W\left(1 \otimes\left(\mathrm{id} \otimes \omega_{\xi, e_{i}}\right)\left(X^{*}\right)\right) .
$$


However, this sum is then equal to

$$
\begin{aligned}
W^{*} \sum_{i}(1 \otimes & \left.\phi\left(\mathrm{id} \otimes \omega_{e_{i}, \eta}\right)(V)\right) W\left(1 \otimes \phi\left(\mathrm{id} \otimes \omega_{\xi, e_{i}}\right)\left(V^{*}\right)\right) \\
& =\sum_{i}(\mathrm{id} \otimes \phi) \Delta_{\mathbb{A P}}\left(\left(\mathrm{id} \otimes \omega_{e_{i}, \eta}\right)(V)\right)\left(1 \otimes \phi\left(\mathrm{id} \otimes \omega_{\xi, e_{i}}\right)\left(V^{*}\right)\right) \\
& =(\mathrm{id} \otimes \phi)\left(\sum_{i} \Delta_{\mathbb{A} \mathbb{P}}\left(\left(\mathrm{id} \otimes \omega_{e_{i}, \eta}\right)(V)\right)\left(1 \otimes \phi\left(\mathrm{id} \otimes \omega_{\xi, e_{i}}\right)\left(V^{*}\right)\right)\right) \\
& =(\mathrm{id} \otimes \phi)\left(\sum_{i}\left(\mathrm{id} \otimes \mathrm{id} \otimes \omega_{e_{i}, \eta}\right)\left(V_{13} V_{23}\right)\left(1 \otimes \phi\left(\mathrm{id} \otimes \omega_{\xi, e_{i}}\right)\left(V^{*}\right)\right)\right) \\
& =(\mathrm{id} \otimes \phi)\left(\sum_{i}\left(\mathrm{id} \otimes \mathrm{id} \otimes \omega_{\xi, \eta}\right)\left(V_{13} V_{23}\left(1 \otimes 1 \otimes \theta_{e_{i}, e_{i}}\right) V_{23}^{*}\right)\right) \\
& =(\mathrm{id} \otimes \phi)\left(\left(\mathrm{id} \otimes \mathrm{id} \otimes \omega_{\xi, \eta}\right)\left(V_{13}\right)\right)=\left(\mathrm{id} \otimes \omega_{\xi, \eta}\right)(V) \otimes 1 .
\end{aligned}
$$

In the final part, of course $V$ is unitary on all of $H_{c}$, not just $H_{c c}$. Thus we have shown that (id $\left.\otimes \omega\right)(Y)=$ $($ id $\otimes \omega)(V)$ for all $\omega \in \mathcal{B}\left(H_{c}\right)_{*}$.

Consider the (non-degenerate) inclusions

$$
\mathbb{A} \mathbb{P}\left(C_{0}^{u}(\mathbb{G})\right) \otimes \mathcal{B}_{0}\left(H_{c}\right) \longrightarrow M\left(C_{0}^{u}(\mathbb{G})\right) \otimes \mathcal{B}_{0}\left(H_{c}\right) \longrightarrow M\left(C_{0}^{u}(\mathbb{G}) \otimes \mathcal{B}_{0}\left(H_{c}\right)\right)
$$

Call the composition $\pi$, so we get the strict extension $\tilde{\pi}: M\left(\mathbb{A P}\left(C_{0}(\mathbb{G})\right) \otimes \mathcal{B}_{0}\left(H_{c}\right)\right) \rightarrow M\left(C_{0}(\mathbb{G}) \otimes \mathcal{B}_{0}\left(H_{c}\right)\right)$. This is injective, as if $\tilde{\pi}(x)=0$ then $\pi(x a)=0$ for all $a \in \mathbb{A P}\left(C_{0}^{u}(\mathbb{G})\right) \otimes \mathcal{B}_{0}\left(H_{c}\right)$ and as $\pi$ is an inclusion, hence $x a=0$ for all $a$, so $x=0$.

Proposition 6.7. With the above notation, we have that $\tilde{\pi}(Y)=V$.

Proof. Let $x \in \mathbb{A P}\left(C_{0}^{u}(\mathbb{G})\right), a \in C_{0}^{u}(\mathbb{G})$ and $\theta_{1}, \theta_{2} \in \mathcal{B}_{0}\left(H_{c}\right)$. We need to show that

$$
\tilde{\pi}(Y) \pi\left(x \otimes \theta_{1}\right)\left(a \otimes \theta_{2}\right)=\pi\left(Y\left(x \otimes \theta_{1}\right)\right)\left(a \otimes \theta_{2}\right) \quad \text { and } \quad V \pi\left(x \otimes \theta_{1}\right)\left(a \otimes \theta_{2}\right)=V\left(x a \otimes \theta_{1} \theta_{2}\right),
$$

agree; similarly there is an analogous argument "on the left". Now, both these are members of $C_{0}^{u}(\mathbb{G}) \otimes$ $\mathcal{B}_{0}\left(H_{c}\right)$, so we can prove equality by slicing by $\omega \in \mathcal{B}\left(H_{c}\right)_{*}$. However,

$$
\begin{aligned}
(\mathrm{id} \otimes \omega)\left(\pi\left(Y\left(x \otimes \theta_{1}\right)\right)\left(a \otimes \theta_{2}\right)\right) & =\left(\mathrm{id} \otimes \theta_{2} \omega\right)\left(\pi\left(Y\left(x \otimes \theta_{1}\right)\right)\right) a=\left(\mathrm{id} \otimes \theta_{2} \omega\right)\left(Y\left(x \otimes \theta_{1}\right)\right) a \\
& =\left(\left(\mathrm{id} \otimes \theta_{1} \theta_{2} \omega\right)(Y) x\right) a=\left(\left(\mathrm{id} \otimes \theta_{1} \theta_{2} \omega\right)(V) x\right) a \\
& =(\mathrm{id} \otimes \omega)\left(V\left(x a \otimes \theta_{1} \theta_{2}\right)\right),
\end{aligned}
$$

as required.

Corollary 6.8. $\pi$ is non-degenerate: $H_{c c}=H_{c}$.

Let $\iota: \mathbb{A P}\left(C_{0}^{u}(\mathbb{G})\right) \rightarrow M\left(C_{0}^{u}(\mathbb{G})\right)$ be the inclusion, which is a quantum group morphism. For any corepresentation $Z \in M\left(\mathbb{A P}\left(C_{0}^{u}(\mathbb{G})\right) \otimes \mathcal{B}_{0}(K)\right)$, the canonical way to induce a corepresentation of $C_{0}^{u}(\mathbb{G})$ is by looking at $(\iota \otimes \mathrm{id})(Z)$. If we unpack this, then $\iota \otimes \mathrm{id}: M\left(\mathbb{A P}\left(C_{0}^{u}(\mathbb{G})\right) \otimes \mathcal{B}_{0}(K)\right) \rightarrow M\left(C_{0}^{u}(\mathbb{G}) \otimes \mathcal{B}_{0}(K)\right)$ is precisely the map $\pi$ above.

So, in conclusion, $V$ arises from a unitary corepresentation, $Y$, of the compact quantum group $\mathbb{A P}\left(C_{0}^{u}(\mathbb{G})\right)$, in the canonical way. As $Y$ decomposes as a direct sum of finite-dimensional corepresentations, the same is hence true of $V$. As $\mathbb{A P}\left(C_{0}^{u}(\mathbb{G})\right)$ is compact, these are also automatically admissible in Soltan's sense. So we have proved the following.

Theorem 6.9. Let $U$ be a unitary corepresentation of $\mathbb{G}$. Then $U$ has a finite-dimensional, admissible sub-corepresentations if and only if $U$ has non-zero compact vectors. 


\section{Decompositions}

For a locally compact quantum group, let $S$ be the antipode, and $R$ the unitary antipode, here supposed to act on $C_{0}^{u}(\mathbb{G})$, see [23, Sections 7 and 9]. In this section we restrict to Kac algebras, or slightly more generally, to those locally compact quantum groups where the antipode and the unitary antipode agree. In this case, the universal corepresentation $\mathcal{W} \in M\left(C_{0}^{u}(\mathbb{G}) \otimes C_{0}^{u}(\widehat{\mathbb{G}})\right)$ generates a $\mathrm{C}^{*}$-Eberelin algebra. Indeed, the coproduct structure on the dual quantum group $C_{0}^{u}(\widehat{\mathbb{G}})$ can be used to show that $B_{0}=\left\{(\operatorname{id} \otimes \widehat{\mu})(\mathcal{W}): \widehat{\mu} \in C_{0}^{u}(\widehat{\mathbb{G}})^{*}\right\}$ is an algebra. As $R=S$, from the usual formula (see [23, Remark 9.1]) we get

$$
R((\operatorname{id} \otimes \widehat{\mu})(\mathcal{W}))=(\operatorname{id} \otimes \widehat{\mu})\left(\mathcal{W}^{*}\right) .
$$

For any corepresentation $U$ let $U^{c}=(R \otimes \top)(U)$ be the contragradient corepresentation (see [37, Proposition 10]), recalling the tranpose map $\top$ from Section 2, Then both $R$ and $\top$ are anti-*-homomorphisms, and so $(R \otimes \top)(U)$ is well-defined. Furthermore, we see that

$$
\left(\mathrm{id} \otimes \omega_{\bar{\xi}, \bar{\eta}}\right)\left(U^{c}\right)=R\left(\left(\mathrm{id} \otimes \omega_{\eta, \xi}\right)(U)\right)=\left(\mathrm{id} \otimes \omega_{\eta, \xi}\right)\left(U^{*}\right)=\left(\mathrm{id} \otimes \omega_{\xi, \eta}\right)(U)^{*} .
$$

So slices of $U^{c}$ give the adjoints of slices of $U$. As $\mathcal{W}$ is the universal corepresentation, slices of $\mathcal{W}^{c}$ form a subset of slices of $\mathcal{W}$. It follows that $B_{0}^{*} \subseteq B_{0}$ and hence actually $B_{0}$ is $*$-closed, and hence its closure $B$ is a $\mathrm{C}^{*}$-algebra. Again by universality, clearly $E(\mathbb{G})=B$.

Now let $U$ be a corepresentation of $C_{0}^{u}(\mathbb{G})$ on $H$. That $\mathcal{W}$ is universal means that $(\mathrm{id} \otimes \omega)(U) \in E(\mathbb{G})$ for any $\omega \in \mathcal{B}(H)_{*}$. With $M$ the (unique) invariant mean on $E(\mathbb{G})$ it thus makes sense to define $p=$ $(M \otimes \mathrm{id})(U)$, as by definition $(p \xi \mid \eta)=\left\langle M,\left(\mathrm{id} \otimes \omega_{\xi, \eta}\right)(U)\right\rangle$ for $\xi, \eta \in H$. We can now copy the start of the proof of Theorem 4.7, carefully noting that we don't need $U$ to generate a $\mathrm{C}^{*}$-Eberlein algebra, only that slices of $U$ are in $E(\mathbb{G})$. Thus we may conclude that $p$ is the orthogonal projection onto $\operatorname{Inv}(U)$ and so $\left\langle M,\left(\mathrm{id} \otimes \omega_{\xi, \eta}\right)(U)\right\rangle 1=\left(\mathrm{id} \otimes \omega_{p \xi, \eta}\right)(U)$.

Lemma 7.1. Let $U$ be a corepresentation of $C_{0}^{u}(\mathbb{G})$ on $H$, and consider the corepresentation $U^{c} \oplus U$ on $\bar{H} \otimes H$. Let $p$ be the orthogonal projection onto $\operatorname{Inv}\left(U^{c} \oplus U\right)$. For $x=\left(\mathrm{id} \otimes \omega_{\xi, \xi}\right)(U)$ we have that $\left\langle M, x^{*} x\right\rangle=0$ if and only if $p(\bar{\xi} \otimes \xi)=0$.

Proof. We see that

$$
x^{*} x=\left(\mathrm{id} \otimes \omega_{\bar{\xi}, \bar{\xi}}\right)\left(U^{c}\right)\left(\mathrm{id} \otimes \omega_{\xi, \xi}\right)(U)=\left(\mathrm{id} \otimes \omega_{\bar{\xi} \otimes \xi, \bar{\xi} \otimes \xi}\right)\left(U^{c} \oplus U\right) .
$$

and so $\left\langle M, x^{*} x\right\rangle 1=\left(\mathrm{id} \otimes \omega_{p(\bar{\xi} \otimes \xi), \bar{\xi} \otimes \xi}\right)\left(U^{c} \oplus U\right)$. For any state $\mu \in C_{0}^{u}(\mathbb{G})^{*}$, as $p$ is the projection onto $\operatorname{Inv}\left(U^{c} \oplus U\right)$, it follows that $(\mu \otimes \mathrm{id})\left(U^{c} \oplus U\right) p=p$ and so

$$
M\left(x^{*} x\right)=\left\langle\mu,\left(\operatorname{id} \otimes \omega_{p(\bar{\xi} \otimes \xi), \bar{\xi} \otimes \xi}\right)\left(U^{c} \oplus U\right)\right\rangle=(p(\bar{\xi} \otimes \xi) \mid \bar{\xi} \otimes \xi)=\|p(\bar{\xi} \otimes \xi)\|^{2},
$$

from which the result follows.

We remark that the Cauchy-Schwarz inequality, and polar decomposition, show easily that $\left\langle M, x^{*} x\right\rangle=$ 0 if and only if $\langle M,|x|\rangle=0$.

Motivated by the above, we wish to study when $\operatorname{Inv}\left(U^{c} \oplus U\right)$ is non-trivial.

Proposition 7.2. For any corepresentation $U$, we have that $\operatorname{Inv}\left(U^{c} \oplus U\right) \neq\{0\}$ if and only if $U$ has a finite-dimensional sub-corepresentation.

Proof. This is stated for discrete Kac algebras in [26, Theorem 2.6], but the proof readily generalises.

The following is a generalisation of Godement's decomposition theorem, [17, Theorem 16, page 64].

Theorem 7.3. Let $U$ be a corepresentation of $C_{0}^{u}(\mathbb{G})$. As in Section 6.1, $U=U_{c} \oplus U_{0}$ on $H=H_{c} \oplus H_{c}^{\perp}$ where $H_{c}$ is the subspace of compact vectors. Then $U_{c}$ is canonically induced by a corepresentation of $\mathbb{A P}\left(C_{0}^{u}(\mathbb{G})\right)$, and for any $x=(\mathrm{id} \otimes \omega)\left(U_{0}\right)$, we have that $\left\langle M, x^{*} x\right\rangle=0$. 
Proof. Given the results of Section 6.1 all we have to show is that $\left\langle M, x^{*} x\right\rangle=0$ for $x=($ id $\otimes \omega)\left(U_{0}\right)$. By Lemma 6.4, we know that $U_{0}$ has no non-zero compact vectors. By replacing $H_{c}^{\perp}$ with $H_{c}^{\perp} \otimes \ell^{2}$ we may suppose that $\omega=\omega_{\xi, \eta}$; notice that $H_{c}^{\perp} \otimes \ell^{2}$ also has no non-zero compact vectors, as if $\sum_{i} \xi_{i} \otimes \delta_{i}$ is compact then slicing against $\eta \otimes \delta_{j}$ for a fixed $j$ shows that $\xi_{j}$ is compact, so $\xi_{j}=0$.

As in the proof of Lemma 7.1 , we look at

$$
\left\langle M, x^{*} x\right\rangle=\left\langle M,\left(\mathrm{id} \otimes \omega_{\bar{\xi} \otimes \xi, \bar{\eta} \otimes \eta}\right)\left(U_{0}^{c} \oplus U_{0}\right)\right\rangle=\left\langle\mu \otimes \omega_{p(\bar{\xi} \otimes \xi), \bar{\eta} \otimes \eta}, U_{0}^{c} \oplus U_{0}\right\rangle
$$

which holds for any state $\mu$, with again $p$ the orthogonal projection onto $\operatorname{Inv}\left(U_{0}^{c} \oplus U_{0}\right)$. As $U_{0}$ has no non-zero compact vectors, Theorem 6.9 shows that $U_{0}$ has no finite-dimensional (admissible) subcorepresentations, and so Proposition 7.2 shows that $\operatorname{Inv}\left(U_{0}^{c} \oplus U_{0}\right)=\{0\}$. Thus $p=0$ and so $\left\langle M, x^{*} x\right\rangle=0$ as claimed.

A similar result is the following.

Proposition 7.4. Let $U$ be a corepresentation of $\mathbb{G}$. Then $U$ has no compact vectors if and only if $\langle M,|x|\rangle=0$ for all $x=(\mathrm{id} \otimes \omega)(U)$.

Proof. As $\left\langle M, x^{*} x\right\rangle=0$ if and only if $\langle M,|x|\rangle=0$, given the theorem, we need only show that if $U$ has compact vectors, then for some choice of $x=(\mathrm{id} \otimes \omega)(U)$ we have that $\left\langle M, x^{*} x\right\rangle \neq 0$. By Theorem 6.9, in this case $U$ admits a finite-dimensional sub-corepresentation, say

$$
V=\sum_{i, j=1}^{n} v_{i, j} \otimes e_{i, j}
$$

where $U$ acts on $H$, where $\left(e_{i}\right)_{i=1}^{n}$ is an orthonormal subset of $H$, and where $\left(e_{i, j}\right)$ are the matrix units associated with $\left(e_{i}\right)$. So $e_{i, j}$ is the rank-one operator sending $e_{j}$ to $e_{i}$.

That $V$ is unitary means that $\sum_{i} v_{i, j}^{*} v_{i, j}=1$ for all $j$, and so $1=\langle M, 1\rangle=\sum_{i}\left\langle M, v_{i, j}^{*} v_{i, j}\right\rangle$. Thus there is some choice of $i, j$ with $\left\langle M, v_{i, j}^{*} v_{i, j}\right\rangle \neq 0$. Then we compute that $\left(\mathrm{id} \otimes \omega_{e_{j}, e_{i}}\right)(U)=v_{i, j}$, and so $x=\left(\mathrm{id} \otimes \omega_{e_{j}, e_{i}}\right)(U)=v_{i, j}$ is a suitable choice to give $\left\langle M, x^{*} x\right\rangle \neq 0$ as required.

\subsection{At the algebra level}

For a locally compact group $G$, we have that $\mathcal{E}(G)=A P\left(C_{0}(G)\right) \oplus \mathcal{E}_{0}(G)$, this usually being proved by compact semigroup considerations, for example [4, Chapter 4]. In this section, we explore this for Kac algebras, and in particular, give a direct $C^{*}$-algebraic proof in the group case.

Proposition 7.5. Let $\mathbb{G}$ be a Kac algebra and let $M$ be the invariant mean on $\mathcal{E}(\mathbb{G})$. Then $M$ is a trace.

Proof. Let $U$ be a corepresentation of $\mathbb{G}$ on $H$, so by Theorem 7.3 , we have that $U=U_{c} \oplus U_{0}$ with respect to $H=H_{c} \oplus H_{0}$. Let $\xi=\xi_{c}+\xi_{0}, \eta=\eta_{c}+\eta_{0}$ with respect to this orthogonal decomposition, and set

$$
x=\left(\mathrm{id} \otimes \omega_{\xi, \eta}\right)(U)=\left(\mathrm{id} \otimes \omega_{\xi_{c}, \eta_{c}}\right)\left(U_{c}\right)+\left(\mathrm{id} \otimes \omega_{\xi_{0}, \eta_{0}}\right)\left(U_{0}\right)=x_{c}+x_{0}
$$

say, where $x_{c} \in \mathbb{A P}\left(C_{0}^{u}(\mathbb{G})\right)$ and $\left\langle M, x_{0}^{*} x_{0}\right\rangle=0$. By Cauchy-Schwarz, also $\left\langle M, x_{0}^{*} x_{c}\right\rangle=\left\langle M, x_{c}^{*} x_{0}\right\rangle=0$ and so $\left\langle M, x^{*} x\right\rangle=\left\langle M, x_{c}^{*} x_{c}\right\rangle$. As $x^{*}$ is a slice of $U^{c}$, by applying the same argument to $U^{c}$, we conclude also that $\left\langle M, x x^{*}\right\rangle=\left\langle M, x_{c} x_{c}^{*}\right\rangle$.

The inclusion $\mathbb{A P}\left(C_{0}^{u}(\mathbb{G})\right) \rightarrow M\left(C_{0}^{u}(\mathbb{G})\right)$ intertwines the coproducts. Let $\left(\tau_{t}\right)$ be the scaling group of $\mathbb{A P}\left(C_{0}^{u}(\mathbb{G})\right)$, so by [23, Remark 12.1], the inclusion intertwines $\tau_{t}$ and the scaling group of $M\left(C_{0}^{u}(\mathbb{G})\right)$, which is of course trivial as $\mathbb{G}$ is Kac. It follows that $\tau_{t}=\mathrm{id}$ for all $t$, and so $\mathbb{A P}\left(C_{0}^{u}(\mathbb{G})\right)$ is a compact Kac algebra.

By Proposition 6.1, $M$ restricted to $\mathbb{A P}\left(C_{0}^{u}(\mathbb{G})\right)$ is the Haar state, and so is a trace on $\mathbb{A P}\left(C_{0}^{u}(\mathbb{G})\right)$. Hence $\left\langle M, x^{*} x\right\rangle=\left\langle M, x_{c}^{*} x_{c}\right\rangle=\left\langle M, x_{c} x_{c}^{*}\right\rangle=\left\langle M, x x^{*}\right\rangle$. As such $x$ are dense in $\mathcal{E}(\mathbb{G})$ the result follows by continuity. 
Proposition 7.6. Let $\mathbb{G}$ be a Kac algebra, let $M$ be the invariant mean on $\mathcal{E}(\mathbb{G})$, and let $\mathcal{E}_{0}(\mathbb{G})=\{x \in$ $\left.\mathcal{E}(\mathbb{G}):\left\langle M, x^{*} x\right\rangle=0\right\}$. Then $\mathcal{E}_{0}(\mathbb{G})$ is a closed two-sided ideal, and with $\left(H, \pi, \xi_{0}\right)$ the GNS triple of $M$, we have that $\mathcal{E}(\mathbb{G}) / \mathcal{E}_{0}(\mathbb{G})=\pi\left(\mathcal{E}(\mathbb{G})\right.$ ). Furthermore, if $\mathbb{A P}\left(C_{0}^{u}(\mathbb{G})\right.$ ) induces the compact quantum group $\mathbb{G}^{\mathrm{SAP}}$ then $H$ is isomorphic to $L^{2}\left(\mathbb{G}^{\mathrm{SAP}}\right)$ in such a way that $\pi(\mathcal{E}(\mathbb{G}))=C\left(\mathbb{G}^{\mathrm{SAP}}\right)$.

Proof. As $M$ is a trace, it follows that $\mathcal{E}_{0}(\mathbb{G})$ is a two-sided ideal, the kernel of $\pi$ is $\mathcal{E}_{0}(\mathbb{G})$, and that hence $\pi(\mathcal{E}(\mathbb{G}))=\mathcal{E}(\mathbb{G}) / \mathcal{E}_{0}(\mathbb{G})$. By the proof of the previous proposition, we also immediately see that $\pi(\mathcal{E}(\mathbb{G}))=\pi\left(\mathbb{A P}\left(C_{0}^{u}(\mathbb{G})\right)\right)$. Now, $L^{2}\left(\mathbb{G}^{\mathrm{SAP}}\right)$ is the GNS space for the Haar state on $\mathbb{A P}\left(C_{0}^{u}(\mathbb{G})\right)$, which is now seen to be isomorphic to $H$, and $C\left(\mathbb{G}^{\mathrm{SAP}}\right)=\pi\left(\mathbb{A P}\left(C_{0}^{u}(\mathbb{G})\right)\right)$ which we have just shown to be equal to $\pi(\mathcal{E}(\mathbb{G}))=\mathcal{E}(\mathbb{G}) / \mathcal{E}_{0}(\mathbb{G})$.

We hence obtain a short exact sequence of $C^{*}$-algebras

$$
0 \rightarrow \mathcal{E}_{0}(\mathbb{G}) \rightarrow \mathcal{E}(\mathbb{G}) \rightarrow C\left(\mathbb{G}^{\mathrm{SAP}}\right) \rightarrow 0
$$

where the first map in inclusion, and the second map is $\pi$.

Theorem 7.7. If $\mathbb{A P}\left(C_{0}^{u}(\mathbb{G})\right)$ is reduced, in particular, if $\mathbb{G}^{\mathrm{SAP}}$ is coamenable, then $\mathcal{E}(\mathbb{G})=\mathcal{E}_{0}(\mathbb{G}) \oplus$ $\mathbb{A P}\left(C_{0}^{u}(\mathbb{G})\right)$.

Proof. By definition, if $\mathbb{A P}\left(C_{0}^{u}(\mathbb{G})\right)$ is reduced then $\pi: \mathbb{A P}\left(C_{0}^{u}(\mathbb{G})\right) \rightarrow C\left(\mathbb{G}^{\mathrm{SAP}}\right)$ is an isomorphism and so there is a map $\iota: C\left(\mathbb{G}^{\mathrm{SAP}}\right) \rightarrow \mathbb{A P}\left(C_{0}^{u}(\mathbb{G})\right)$ with $\pi \circ \iota=\mathrm{id}$. Treating $\iota$ as a map $C\left(\mathbb{G}^{\mathrm{SAP}}\right) \rightarrow \mathcal{E}(\mathbb{G})$, we get a splitting of the short exact sequence, and so $\mathcal{E}(\mathbb{G})$ splits as $\mathcal{E}_{0}(\mathbb{G}) \oplus \mathbb{A P}\left(C_{0}^{u}(\mathbb{G})\right)$. If $\mathbb{G}^{\mathrm{SAP}}$ is coamenable then, compare [2, Theorem 3.1], $\mathbb{A P}\left(C_{0}^{u}(\mathbb{G})\right)$ is necessarily reduced.

This result immediately gives us the claimed result in the group case, as a group is always co-amenable. We shall see below in Section 8.1 that even in the cocommutative case, we need some condition to get such a splitting result.

\section{Compactification examples}

We motivated our approach to compactifications in Section 5 by showing that it agreed with the ideas of Spronk and Stokke in [39]. Indeed, [39, Theorem 3.14] gives a construction of $G^{\mathcal{C H}}$ by showing that $G^{\mathcal{C H}}$ can be realised as the Eberlein compactification of $G$, constructed in [39, Section 3.1]. Namely, let $\omega_{G}: G \rightarrow \mathcal{U}\left(H_{\omega_{G}}\right)$ be the universal (unitary, SOT continuous) representation of $G$, and then set $G^{\mathcal{C H}}$ to be the weak ${ }^{*}$-closure of $\omega_{G}(G)$; notice that by construction $G^{\mathcal{C H}} \in \mathrm{WCH}$.

This is the same as our construction, as Theorem 5.1 above essentially constructs a maximal corepresentation $U$ of $C_{0}(G)$ with the property that slices of $U$ are dense in a $*$-algebra. In Section 7 above we saw that for Kac algebras, the universal corepresentation has this property. As corepresentations of $C_{0}(G)$ biject with representations of $G$, this is the same construction as in [39].

Remark 8.1. In Section 3.3 we showed links with the extended Haagerup tensor product. Suppose $G$ is a locally compact group. It would be tempting to look at functions $F \in L^{\infty}(G)$ with $\Delta(F)$ in the norm closure of $L^{\infty}(G) \otimes^{e h} L^{\infty}(G)$, say giving the collection $E_{e h}(G)$. This would give a $\mathrm{C}^{*}$-algebra which contains $\mathcal{E}(G)$ (by the remarks in Section 3.3) and seems to have many of the properties of $\mathcal{E}(G)$. However, it seems quite possible that this gives something larger than $\mathcal{E}(G)$, even in this classical (i.e. $G$ is a group) setting.

We follow Spronk's paper [38]. Now, $\Delta(F)$ is the function $G \times G \rightarrow \mathbb{C} ;(s, t) \mapsto F(s t)$. As $L^{\infty}(G)$ is commutative, it is not hard to see that the function $u(s, t)=\Delta(F)\left(s, t^{-1}\right)=F\left(s t^{-1}\right)$ will then also be in $L^{\infty}(G) \otimes^{e h} L^{\infty}(G)$. Furthermore, $u$ is invariant in the sense of [38, Section 5] as $u(s r, t)=F\left(s r t^{-1}\right)=$ $F\left(s\left(t r^{-1}\right)^{-1}\right)=u\left(s, t r^{-1}\right)$. Thus $a: G \rightarrow \mathbb{C}$ defined by $a(s t)=u\left(s, t^{-1}\right)=F(s t)$ is a completely bounded multiplier of $A(G)$; but $a=F$ of course. We can reverse this argument (see again [38]) and so we conclude that $E_{e h}(G)$ is precisely the norm closure of $M_{c b} A(G)$ in $L^{\infty}(G)$. However, it is not clear to us why, for arbitrary $G$, this wouldn't be larger than $\mathcal{E}(G)$. 


\subsection{The cocommutative case}

We now study the cocommutative case. Let $G$ be a locally compact group, and set $\mathbb{G}=\hat{G}$ so that $C_{0}^{u}(\mathbb{G})=C^{*}(G)$ the (full) group $\mathrm{C}^{*}$-algebra of $G$. Again, this is a Kac algebra, and so $\mathcal{E}(G)$ is the closure of the image of the measure algebra $M(G)=C_{0}(G)^{*}$ inside $M\left(C^{*}(G)\right)$. We also know that $\mathbb{A P}\left(C^{*}(G)\right)$ is the norm closure of the translation operators $\{\lambda(g): g \in G\}$ inside $M\left(C^{*}(G)\right)$, see [36, Proposition 4.3] or [10, Section 5].

Let us think about compact vectors, and decompositions, in this setting. We shall just sketch these results, leaving the details to be verified by the reader. There is a bijection between corepresentations of $C^{*}(G)$ and *-representations of $C_{0}(G)$. Any *-representation of $C_{0}(G)$ is the direct sum of cyclic representations, and these all have the form of $C_{0}(G)$ acting by multiplication on $L^{2}(G, \mu)$, where $\mu$ is a Borel probability measure on $G$. Let $\pi: C_{0}(G) \rightarrow L^{2}(G, \mu)$ induce the corepresentation $U \in$ $M\left(C^{*}(G) \otimes \mathcal{B}\left(L^{2}(G, \mu)\right)\right)$. For $\xi, \eta \in L^{2}(G, \mu)$ the coefficient $\left(\right.$ id $\left.\otimes \omega_{\xi, \eta}\right)(U) \in \mathcal{E}(\hat{G}) \subseteq M\left(C^{*}(G)\right)$ is the operator given by (left) convolution by the measure $\phi$ where

$$
\int_{G} f(s) d \phi(s)=\int_{G} f\left(s^{-1}\right) \xi(s) \overline{\eta(s)} d \mu(s) .
$$

The $s^{-1}$ occurs because of our choice of conventions: essentially because $\widehat{W}=\sigma W^{*} \sigma$.

We know that $\mathbb{A P}\left(C^{*}(G)\right)$ is equal to the closed linear span of the translation operators $\{\lambda(s): s \in G\}$ in $M\left(C^{*}(G)\right)$; see [36, Section 4.2]. This is a compact quantum group living between $C^{*}\left(G_{d}\right)$ and $C_{r}^{*}\left(G_{d}\right)$, where $G_{d}$ is $G$ with the discrete topology; compare [10, Section 6.2]. Let $U$ be a corepresentation of $\mathbb{A} \mathbb{P}\left(C^{*}(G)\right.$ ) (which always comes from a corepresentation of $C^{*}\left(G_{d}\right)$, so from a $*$-representation $\pi_{d}$ of $c_{0}\left(G_{d}\right)$ ) and let this induce a corepresentation of $C^{*}(G)$, so a *-representation $\pi$ of $C_{0}(G)$ on $H$. Then for $f \in C_{0}(G)$, let this give a function in $M\left(C_{0}\left(G_{d}\right)\right) \cong \ell^{\infty}(G)$, and then $\pi(f)=\pi_{d}(f)$ where we take the strict extension of $\pi_{d}$ to $\ell^{\infty}(G)$. It follows that $\pi$ is equivalent to a sum of "multiplication" representations on $L^{2}(G, \mu)$ where $\mu$ is a purely atomic measure (because this is how $\pi_{d}$ will arise).

So given $\pi: C_{0}(G) \rightarrow \mathcal{B}\left(L^{2}(G, \mu)\right)$ inducing $U$, the decomposition result of Theorem 7.3 induces a decomposition of $\pi$, say as $\pi=\pi_{c}+\pi_{0}$. This comes from decomposing $\mu=\mu_{c}+\mu_{0}$ where $\mu_{c}$ is the atomic part of $\mu$, and $\mu_{0}$ is the non-atomic part, and where $\pi_{c}: C_{0}(G) \rightarrow \mathcal{B}\left(L^{2}\left(G, \mu_{0}\right)\right)$, and similarly for $\pi_{0}$. Then the results of Section 7.1 readily show that if $\mu$ is a measure on $G$, inducing the convolution operator $L_{\mu} \in M\left(C^{*}(G)\right)$, then the invariant mean satisfies $\left\langle M, L_{\mu}\right\rangle=\mu(\{e\})$, so singling out the atomic part at the identity of $e$.

For example, if $G_{d}$ is not amenable, then the canonical map from $\mathbb{A P}\left(C_{0}^{u}(\hat{G})\right) \rightarrow C_{r}^{*}\left(G_{d}\right)$ is not an isomorphism, see [10, Section 6.2]. Thus $M$, restricted to $\mathbb{A P}\left(C_{0}^{u}(\hat{G})\right)$, is not faithful, and so $\mathcal{E}_{0}(\mathbb{G}) \cap$ $\mathbb{A P}\left(C_{0}^{u}(\hat{G})\right)$ is more than just $\{0\}$. The "problem" here is that $\mathbb{A P}\left(C_{0}^{u}(\hat{G})\right)$ is larger than $C_{r}^{*}\left(G_{d}\right)$. However, there can be no map $r: C_{r}^{*}\left(G_{d}\right) \rightarrow \mathbb{A P}\left(C_{0}^{u}(\hat{G})\right)$ with $q \circ r=\mathrm{id}$, where $q: \mathcal{E}(\hat{G}) \rightarrow C_{r}^{*}\left(G_{d}\right)$ is as in Proposition 7.6. Indeed, for any $s \in G$, we have that $q(\lambda(s))=\lambda(s) \in C_{r}^{*}\left(G_{d}\right)$, and so we would need that $r(\lambda(s))=\lambda(s)$. As both $C_{r}^{*}\left(G_{d}\right)$ and $\mathbb{A P}\left(C_{0}^{u}(\hat{G})\right)$ are the closure of $\{\lambda(s): s \in G\}$ in different algebras, this would force $r$ to be have dense range, and have closed range (as a $*$-homomorphism) and hence be onto, a contradiction.

In the literature, it is more usual to work with $C_{r}^{*}(G)$, instead of $C^{*}(G)$, and hence view $M\left(C_{r}^{*}(G)\right)$ as a subalgebra of $V N(G)$. In this context, it is well-known that even wap $(V N(G))$ always has a unique invariant mean, [18, Proposition 5]. Ideas somewhat related to, but put to different ends than, those in this section can be found in [1, 14].

\section{References}

[1] E. Bédos, "On the $C^{*}$-algebra generated by the left regular representation of a locally compact group", Proc. Amer. Math. Soc. 120 (1994) 603-608. 
[2] E. Bédos, L. Tuset, "Amenability and co-amenability for locally compact quantum groups", Internat. J. Math. 14 (2003) 865-884.

[3] R. B. Burckel, "Weakly almost periodic functions on semigroups", (Gordon and Breach Science Publishers, New York-London-Paris, 1970).

[4] J. Berglund, H. Junghenn, P. Milnes, "Analysis on semigroups. Function spaces, compactifications, representations", (John Wiley \& Sons, Inc., New York, 1989).

[5] V. Bergelson, J. Rosenblatt, "Mixing actions of groups." Illinois J. Math. 32 (1988) 65-80.

[6] D. P. Blecher, R. R. Smith, "The dual of the Haagerup tensor product", J. London Math. Soc. 45 (1992) 126-144.

[7] M. Brannan, E. Samei, "The similarity problem for Fourier algebras and corepresentations of group von Neumann algebras", J. Funct. Anal. 259 (2010) 2073-2097.

[8] M. Brannan, M. Daws, E. Samei, "Completely bounded representations of convolution algebras of locally compact quantum groups", Münster J. Math. 6 (2013) 445-482.

[9] J. B. Conway, "A course in functional analysis", (Springer-Verlag, New York, 1990).

[10] M. Daws, "Remarks on the Quantum Bohr Compactification", to appear in Illinois J. Math., see arXiv:1307.1412v2 [math.FA]

[11] M. Daws, "Dual Banach algebras: representations and injectivity", Studia Math. 178 (2007) 231-275.

[12] K. de Leeuw, I. Glicksberg, "Applications of almost periodic compactifications", Acta Math. 105 (1961) 63-97.

[13] J. Dixmier, "C*-algebras". Translated from the French by Francis Jellett. North-Holland Mathematical Library, Vol. 15. (North-Holland Publishing Co., Amsterdam-New York-Oxford, 1977).

[14] C. F. Dunkl, D. E. Ramirez, " $C^{*}$-algebras generated by Fourier-Stieltjes transforms", Trans. Amer. Math. Soc. 164 (1972) 435-441.

[15] E. G. Effros, Z.-J. Ruan, "Operator spaces", (Oxford University Press, New York, 2000).

[16] E. G. Effros, Z.-J. Ruan, "Operator space tensor products and Hopf convolution algebras", J. Operator Theory 50 (2003) 131-156.

[17] R. Godement, "Les fonctions de type positif et la théorie des groupes", Trans. Amer. Math. Soc. 63 (1948) 1-84.

[18] E. E. Granirer, "Weakly almost periodic and uniformly continuous functionals on the Fourier algebra of any locally compact group", Trans. Amer. Math. Soc. 189 (1974) 371-382.

[19] A. Grothendieck, "Critères de compacité dans les espaces fonctionnels généraux", Amer. J. Math. 74 (1952) 168-186.

[20] Z. Hu, M. Neufang, Z.-J. Ruan, "Module maps over locally compact quantum groups", Studia Math. 211 (2012) 111-145.

[21] B. E. Johnson, "Separate continuity and measurability", Proc. Amer. Math. Soc. 20 (1969) 420-422.

[22] J. Kustermans, "Locally compact quantum groups" in Quantum independent increment processes. I, Lecture Notes in Math. 1865, pp. 99-180 (Springer, Berlin, 2005). 
[23] J. Kustermans, "Locally compact quantum groups in the universal setting", Internat. J. Math. 12 (2001) 289-338.

[24] J. Kustermans, S. Vaes, "Locally compact quantum groups in the von Neumann algebraic setting", Math. Scand. 92 (2003) 68-92.

[25] J. Kustermans, S. Vaes, "Locally compact quantum groups", Ann. Sci. École Norm. Soup. 33 (2000) 837-934.

[26] D. Kyed, P. Soltan, "Property (T) and exotic quantum group norms", J. Noncommut. Geom. 6 (2012) 773-800.

[27] A. T.-M. Lau, R. J. Loy, "Weak amenability of Banach algebras on locally compact groups", J. Funct. Anal. 145 (1997) 175-204.

[28] A. Maes, A. Van Daele, "Notes on compact quantum groups", Nieuw Arch. Wisk. 16 (1998) 73-112.

[29] T. Masuda, Y. Nakagami, S. L. Woronowicz, "A $C^{*}$-algebraic framework for quantum groups", Internat. J. Math. 14 (2003) 903-1001.

[30] R. Meyer, S. Roy, S. L. Woronowicz, "Homomorphisms of quantum groups", Münster J. Math. 5 (2012) 1-24.

[31] M. Megrelishvili, "Reflexively representable but not Hilbert representable compact flows and semitopological semigroups", Colloq. Math. 110 (2008) 383-407.

[32] G. Pisier, "Introduction to operator space theory", (Cambridge University Press, Cambridge, 2003).

[33] V. Runde, "Uniform continuity over locally compact quantum groups", J. Lond. Math. Soc. 80 (2009) $55-71$.

[34] V. Runde, "Connes-amenability and normal, virtual diagonals for measure algebras. I.", J. London Math. Soc. 67 (2003) 643-656.

[35] P. Salmi, "Quantum semigroup compactifications and uniform continuity on locally compact quantum groups", Illinois J. Math. 54 (2010) 469-483.

[36] P. Sołtan, "Quantum Bohr Compactification", Illinois J. Math. 49 (2005) 1245-1270.

[37] P. Soltan, S.L. Woronowicz, "From multiplicative unitaries to quantum groups. II", J. Funct. Anal. 252 (2007) 42-67.

[38] N. Spronk, "Measurable Schur multipliers and completely bounded multipliers of the Fourier algebras", Proc. London Math. Soc. 89 (2004) 161-192.

[39] N. Spronk, R. Stokke, "Matrix coefficients of unitary representations and associated compactifications", Indiana Univ. Math. J. 62 (2013) 99-148.

[40] R. Stokke, "Amenable representations and coefficient subspaces of Fourier-Stieltjes algebras", Math. Scand. 98 (2006) 182-200.

[41] S. Vaes, "Locally compact quantum groups", PhD. thesis, Katholieke Universiteit Leuven, 2001. Available from http://wis.kuleuven.be/analyse/stefaan/

[42] S. L. Woronowicz, "Compact quantum groups", in: Symétries quantiques (Les Houches, 1995), 845884 (North-Holland, Amsterdam, 1998). 
Authors's Addresses:

Instytut Matematyczny PAN

8, ul. Śniadeckich

00-656, Warsaw

Poland

School of Mathematics

University of Leeds

Leeds LS2 9JT

United Kingdom

Email: biswarupnow@gmail.com

Email: matt.daws@cantab.net 